\title{
IDENTITIES AND POSITIVITY CONJECTURES FOR SOME REMARKABLE OPERATORS IN THE THEORY OF SYMMETRIC FUNCTIONS*
}

\author{
F. BERGERON ${ }^{\dagger}$, A. M. GARSIA ${ }^{\ddagger}$, M. HAIMAN ${ }^{\ddagger}$, AND G. TESLER $^{\ddagger}$
}

To Richard Askey on his 65th birthday

\begin{abstract}
Let $J_{\mu}[X ; q, t]$ be the integral form of the Macdonald polynomial and set $\tilde{H}_{\mu}[X ; q, t]$ $=t^{n(\mu)} J_{\mu}[X /(1-1 / t) ; q, 1 / t]$, where $n(\mu)=\sum_{i}(i-1) \mu_{i}$. This paper focusses on the linear operator $\nabla$ defined by setting $\nabla \tilde{H}_{\mu}=t^{n(\mu)} q^{n\left(\mu^{\prime}\right)} \tilde{H}_{\mu}$. This operator occurs naturally in the study of the Garsia-Haiman modules $\mathbf{M}_{\mu}$. It was originally introduced by the first two authors to give elegant expressions to Frobenius characteristics of intersections of these modules (see [3]). However, it was soon discovered that it plays a powerful and ubiquitous role throughout the theory of Macdonald polynomials. Our main result here is a proof that $\nabla$ acts integrally on symmetric functions. An important corollary of this result is the Schur integrality of the conjectured Frobenius characteristic of the Diagonal Harmonic polynomials [11]. Another curious aspect of $\nabla$ is that it appears to encode a $q, t$-analogue of Lagrange inversion. In particular, its specialization at $t=1$ (or $q=1$ ) reduces to the $q$-analogue of Lagrange inversion studied by Andrews [1], Garsia [7] and Gessel [17]. We present here a number of positivity conjectures that have emerged in the few years since $\nabla$ has been discovered. We also prove a number of identities in support of these conjectures and state some of the results that illustrate the power of $\nabla$ within the Theory of Macdonald polynomials.
\end{abstract}

Introduction. The study of $\nabla$ and some closely related variants relies on a number of important discoveries, including the introduction of a family of plethystic operators with remarkable properties. This amounts to an extension of Classical Symmetric Function Theory which should have a variety of applications even outside of the Theory of Macdonald polynomials. These developments have been emerging from several published and unpublished works. However, most of what is needed here is given a detailed presentation in the paper "Explicit Plethystic Formulas for Macdonald $q, t$-Kostka coefficients"[13]. The reader is urged to get a copy of that paper as an aid to reading the present one. To avoid unnecessary duplications we shall limit ourselves to giving the most important definitions, stating the basic results and refer the reader to the appropriate sources for the omitted details.

We shall work with the algebra $\Lambda$ of symmetric functions in a formal infinite alphabet $X=x_{1}, x_{2}, \ldots$, with coefficients in the field of rational functions $\mathbf{Q}(q, t)$. We also denote by $\Lambda_{Z[q, t]}$ the algebra of symmetric functions in $X$ with coefficients in $Z[q, t]$. The space $\Lambda_{Z[q, t, 1 / q, 1 / t]}$ is analogously defined. We write $\Lambda^{=d}$ for the space of symmetric functions homogeneous of degree $d$. Similarly we define $\Lambda \leq d$ and $\Lambda^{>d}$. We shall make extensive use here of "plethystic" notation and we need to recall its definition. Briefly, if $E=E\left(t_{1}, t_{2}, t_{3}, \ldots\right)$ is a given formal series in the variables

* Received August 31, 1999.

† Mathematics Department, Université du Québec à Montréal, Montréal, Québec, Canada (bergeron@lacim.uqam.ca). Work carried out under NSERC and FCAR grant support.

$\ddagger$ Mathematics Department, University of California, San Diego, La Jolla, CA 92093, USA (garsia@schur.ucsd.edu). Work carried out under NSF grant support. 
$t_{1}, t_{2}, t_{3}, \ldots$ (which may include the parameters $q, t$ ) and $f \in \Lambda$ has been expressed in terms of the power basis in the form

$$
f=Q\left(p_{1}, p_{2}, p_{3}, \ldots\right)
$$

then the "plethystic substitution" of $E$ in $f$, denoted $f[E]$, is simply defined by setting

$$
F[E]=\left.Q\left(p_{1}, p_{2}, p_{3}, \ldots\right)\right|_{p_{k} \rightarrow E\left(t_{1}^{k}, t_{2}^{k}, t_{3}^{k}, \ldots\right)} .
$$

This operation is easily programmed in any symbolic manipulation software which includes a symmetric function package. It is also very convenient to express, in a compact form, many of the basic identities of Symmetric Function Theory.

We shall adopt the convention that inside the plethystic brackets "[ ]", $X$ and $X_{n}$ respectively stand for $x_{1}+x_{2}+x_{3}+\cdots$ and $x_{1}+x_{2}+\cdots+x_{n}$. We also need to introduce a plethystic notation for the customary operation of replacing variables by their negatives. This is to be distinguished from the operation resulting from the "plethystic" minus sign. We will represent the former operation in two ways. We may prepend the variable in question by a superscripted minus sign or we may multiply it by a symbolic " -1 " which for convenience will also be denoted by " $\epsilon$ ". For example, the definition in $I .1$ requires that

$$
p_{k}\left[-X_{n}\right]=-\left(x_{1}^{k}+x_{2}^{k}+\cdots+x_{n}^{k}\right)
$$

while this additional notation yields

$p_{k}\left[{ }^{-} X_{n}\right]=p_{k}\left[{ }^{-} 1 \times X_{n}\right]=p_{k}\left[\epsilon X_{n}\right]=\left.p_{k}\left[X_{n}\right]\right|_{x_{i} \rightarrow-x_{i}}=(-1)^{k}\left(x_{1}^{k}+x_{2}^{k}+\cdots+x_{n}^{k}\right)$.

From this we easily deduce that the fundamental involution " $\omega$ " acting on a symmetric polynomial $P$ of degree $\leq n$ may be expressed in the form

$$
\omega P\left[X_{n}\right]=P\left[-^{-} X_{n}\right] .
$$

Partitions will be represented and identified with their "french" Ferrers diagrams. Given a partition $\mu=\left(\mu_{1} \geq \mu_{2} \geq \cdots \geq \mu_{k}>0\right)$, we let the corresponding Ferrers diagram have $\mu_{i}$ lattice cells in the $i^{\text {th }}$ row (from the bottom up). It will be convenient to let $|\mu|$ and $l(\mu)$ denote respectively the sum of the parts and the number of parts of $\mu$. In this case $|\mu|=\mu_{1}+\mu_{2}+\cdots+\mu_{k}$ and $l(\mu)=k$. As customary the symbol " $\mu \vdash n$ " will be used to indicate that $|\mu|=n$. We shall also adopt the Macdonald convention of calling the arm, leg, coarm and coleg of a lattice square $s$ the parameters $a_{\mu}(s), l_{\mu}(s), a_{\mu}^{\prime}(s)$ and $l_{\mu}^{\prime}(s)$ giving the number of cells of $\mu$ that are respectively strictly EAST, NORTH, WEST and SOUTH of $s$ in $\mu$.

We set

$$
n(\mu)=\sum_{i=1}^{n}(i-1) \mu_{i}=\sum_{s \in \mu} l_{\mu}^{\prime}(s)=\sum_{s \in \mu} l_{\mu}(s) .
$$

If $s$ is a cell of $\mu$ we shall refer to the monomial $w(s)=q^{a_{\mu}^{\prime}(s)} t^{l_{\mu}^{\prime}(s)}$ as the weight of $s$. We also set

$$
E_{\mu}^{\prime}(q, t)=\sum_{s \in \mu} q^{a_{\mu}^{\prime}(s)} t^{l_{\mu}^{\prime}(s)} \quad \text { and } \quad \Pi_{\mu}(q, t)=\prod_{s \in \mu /(0,0)}\left(1-q^{a_{\mu}^{\prime}(s)} t^{l_{\mu}^{\prime}(s)}\right)
$$


It will be also convenient to set

$$
\left\{\begin{array}{l}
T_{\mu}=t^{n(\mu)} q^{n\left(\mu^{\prime}\right)}=\prod_{s \in \mu} q^{a_{\mu}^{\prime}(s)} t^{l_{\mu}^{\prime}(s)} \\
D_{\mu}=(1-t)(1-q) B_{\mu}(q, t)-1
\end{array}\right.
$$

This given, our operator $\nabla$ is defined by setting

$$
\nabla \tilde{H}_{\mu}=T_{\mu} \tilde{H}_{\mu}
$$

with

$$
\tilde{H}_{\mu}[X ; q, t]=t^{n(\mu)} J_{\mu}\left[\frac{X}{1-1 / t} ; q, 1 / t\right]
$$

where $J_{\mu}[X ; q, t]$ is the "integral form" introduced by Macdonald in [22. ch. IV (8.3)]. Note that from I. 6 we derive the Schur function expansion

$$
\tilde{H}_{\mu}[X ; q, t]=\sum_{\lambda} S_{\lambda}[X] \tilde{K}_{\lambda \mu}(q, t)
$$

where $\tilde{K}_{\lambda \mu}(q, t)=t^{n(\mu)} K_{\lambda \mu}(q, 1 / t)$ with $K_{\lambda \mu}(q, t)$ the Macdonald $q, t$-Kostka coefficient. We should point out that in this paper $S_{\lambda}[X]$ denotes the the ordinary Schur Function indexed by $\lambda$. By contrast, in Macdonald's book [22], the symbol $S_{\lambda}[X]$ represents what we would denote here by " $S_{\lambda}[X(1-t)]$ "

The operator $\nabla$ played a crucial role in developments relating Macdonald polynomials to Representation Theory [6], [8], [12] and to Geometry [5],[19]. Computer experimentation with $\nabla$ revealed that it has some truly remarkable properties. In this paper we present a collection of results and conjectures about $\nabla$ that have emerged in the few years since its discovery. As a matter of example it is worthwhile having a look at the following beautiful matrices which express the action of $\nabla$ on Schur functions indexed by partitions of 4 : 


$$
\begin{aligned}
& \begin{array}{lllll}
S_{4} & S_{31} & S_{22} & S_{211} & S_{1111}
\end{array} \\
& \nabla S_{4} \rightarrow \quad 0 \quad-t^{3} q^{3} \quad-t^{3} q^{3}\left[\begin{array}{ll}
1 & 0 \\
0 & 1
\end{array}\right] \quad-t^{3} q^{3}\left[\begin{array}{lll}
1 & 0 & 0 \\
1 & 1 & 0 \\
0 & 1 & 1
\end{array}\right] \quad-t^{3} q^{3}\left[\begin{array}{llll}
1 & 0 & 0 & 0 \\
0 & 1 & 0 & 0 \\
0 & 1 & 1 & 0 \\
0 & 0 & 0 & 1
\end{array}\right] \\
& \nabla S_{31} \rightarrow 0 \quad t^{2} q^{2}\left[\begin{array}{ll}
1 & 0 \\
0 & 1
\end{array}\right] \quad t^{2} q^{2}\left[\begin{array}{lll}
1 & 0 & 0 \\
0 & 1 & 0 \\
0 & 0 & 1
\end{array}\right] \quad t^{2} q^{2}\left[\begin{array}{llll}
1 & 0 & 0 & 0 \\
1 & 1 & 0 & 0 \\
0 & 2 & 1 & 0 \\
0 & 0 & 1 & 1
\end{array}\right] \quad t^{2} q^{2}\left[\begin{array}{lllll}
1 & 0 & 0 & 0 & 0 \\
0 & 1 & 0 & 0 & 0 \\
0 & 1 & 1 & 0 & 0 \\
0 & 0 & 1 & 1 & 0 \\
0 & 0 & 0 & 0 & 1
\end{array}\right] \\
& \nabla S_{22} \rightarrow \quad 0 \quad-t^{2} q^{2} \quad 0 \quad-t^{2} q^{2}\left[\begin{array}{ll}
1 & 0 \\
0 & 1
\end{array}\right] \quad-t^{3} q^{3} \\
& \nabla S_{211} \rightarrow 0-t q\left[\begin{array}{lll}
1 & 0 & 0 \\
0 & 1 & 0 \\
0 & 0 & 1
\end{array}\right]-t q\left[\begin{array}{llll}
1 & 0 & 0 & 0 \\
0 & 1 & 0 & 0 \\
0 & 1 & 1 & 0 \\
0 & 0 & 0 & 1
\end{array}\right]-t q\left[\begin{array}{lllll}
1 & 0 & 0 & 0 & 0 \\
1 & 1 & 0 & 0 & 0 \\
0 & 2 & 1 & 0 & 0 \\
0 & 0 & 2 & 1 & 0 \\
0 & 0 & 0 & 1 & 1
\end{array}\right]-t q\left[\begin{array}{llllll}
1 & 0 & 0 & 0 & 0 & 0 \\
0 & 1 & 0 & 0 & 0 & 0 \\
0 & 1 & 1 & 0 & 0 & 0 \\
0 & 0 & 1 & 1 & 0 & 0 \\
0 & 0 & 0 & 1 & 1 & 0 \\
0 & 0 & 0 & 0 & 0 & 1
\end{array}\right] \\
& \nabla S_{1111} \rightarrow 1\left[\begin{array}{llll}
1 & 0 & 0 & 0 \\
1 & 1 & 0 & 0 \\
1 & 1 & 1 & 0 \\
0 & 1 & 1 & 1
\end{array}\right] \quad\left[\begin{array}{lllll}
1 & 0 & 0 & 0 & 0 \\
0 & 1 & 0 & 0 & 0 \\
1 & 1 & 1 & 0 & 0 \\
0 & 1 & 1 & 1 & 0 \\
0 & 0 & 1 & 0 & 1
\end{array}\right] \quad\left[\begin{array}{llllll}
1 & 0 & 0 & 0 & 0 & 0 \\
1 & 1 & 0 & 0 & 0 & 0 \\
1 & 2 & 1 & 0 & 0 & 0 \\
0 & 2 & 2 & 1 & 0 & 0 \\
0 & 1 & 2 & 2 & 1 & 0 \\
0 & 0 & 0 & 1 & 1 & 1
\end{array}\right] \quad\left[\begin{array}{lllllll}
1 & 0 & 0 & 0 & 0 & 0 & 0 \\
0 & 1 & 0 & 0 & 0 & 0 & 0 \\
0 & 1 & 1 & 0 & 0 & 0 & 0 \\
0 & 1 & 1 & 1 & 0 & 0 & 0 \\
0 & 0 & 1 & 1 & 1 & 0 & 0 \\
0 & 0 & 0 & 1 & 1 & 1 & 0 \\
0 & 0 & 0 & 0 & 0 & 0 & 1
\end{array}\right]
\end{aligned}
$$

Here at the intersection of the row indexed by $\nabla S_{\mu}$ with column indexed by $S_{\lambda}$ we have placed the coefficient of $S_{\lambda}$ in $\nabla S_{\mu}$. These coefficients are depicted by the convention that represents the polynomial

$$
t^{2} q^{3}\left(a+b q+c q^{2}+d t+e t q+f t^{2}\right)
$$

by means of the symbol

$$
t^{2} q^{3}\left[\begin{array}{lll}
f & 0 & 0 \\
d & e & 0 \\
a & b & c
\end{array}\right]
$$

A close inspection of these matrices suggests a number of remarkable properties of the image of a Schur function by $\nabla$. To begin with we might infer that $\nabla$ of a Schur function is always totally Schur-positive or totally Schur negative. We also might recognize that the coefficient of $S_{1^{4}}$ in $\nabla S_{1^{4}}$ is the $q, t$-Catalan $C_{4}(q, t)$ studied in [11]. Similarly, we discover that, up to a factor, the coefficient of $S_{1^{4}}$ in $\nabla S_{4}$ is $C_{3}(q, t)$. Now the first observation leads to a conjecture and the second is a particular case of a general theorem that will be proved here. More precisely, we have the following:

CONJECTURE I. For any pair of partitions $\lambda, \mu$ and for a every positive integer $m$ we have

$$
(-1)^{\iota\left(\lambda^{\prime}\right)}\left\langle\nabla^{m} S_{\lambda}, S_{\mu}\right\rangle \in \mathbf{N}[q, t]
$$


with $\langle$,$\rangle the Hall inner product and$

$$
\iota(\lambda)=\left(\begin{array}{c}
l(\lambda) \\
2
\end{array}\right)+\sum_{\lambda_{i}<(i-1)}\left(i-1-\lambda_{i}\right) .
$$

We should mention that the sign in I.8 was identified by M. Bousquet-Melou [4] who gave a combinatorial interpretation to the left hand side of I.8 in the special case $\mu=1^{n}$. We should add that in the case $\mu=1^{n}$ there is a more explicit conjecture. More precisely, it was conjectured in [11] that the bigraded Frobenius characteristic $D H_{n}[X ; q, t]$ of the Diagonal Harmonics (rewritten in terms of $\nabla$ ), is given by the formula

$$
D H_{n}[X ; q, t]=\nabla e_{n},
$$

in fact, the simplicity of this expression for $D H_{n}[X ; q, t]$ was one of the original motivations of the first two authors for introducing this operator. We should also mention that the Schur-positivity of all the successive powers of $\nabla$ on $e_{n}$ was conjectured in [19] to have also a representation theoretical explanation.

A variety of other beautiful identities and positivities have been discovered through computer experimentation. Some of them can actually be proved but others appear out of reach to this date. All of these results have remained unpublished for a number of years since no tools have been available, until recently, to allow a direct study of $\nabla$, other than the Macdonald polynomials, which themselves are fraught with unsolved difficult conjectures.

The specialization of $\nabla$ at $t=1$, denoted " $\nabla_{t=1}$ ", can be shown to be a multiplicative operator closely related to the $q$-Lagrange inversion problem studied in [7]. This connection, which has already been pointed out in [11], shows in particular that a very recent result of $C$. Lenart [20] is none other but a proof of the special case $m=1$ and $t, q=1$ of Conjecture $\mathrm{I}$.

Our main result here is a proof that $\nabla$ acts integrally on Schur functions. In particular, by way of the identity in I.9, we obtain a proof that $D H_{n}[X ; q, t]$ is in fact a Schur integral polynomial and, a fortiori, we now have an elementary proof that the elusive $q, t$-Catalan $c_{n}(q, t)$ of [11] is a polynomial with integer coefficients.

Our experience is that almost every expression or identity that arises in the connection between Macdonald polynomials and Representation Theory may be simply formulated in terms of $\nabla$.

Our proof of the polynomiality of $\nabla$ hinges on the development of a theory of plethystic operators which promises to play a central role in the theory of symmetric functions. The basic ingredients of this theory are operators $D_{k}$ and $D_{k}^{*}$ defined for any integer $-\infty<k<+\infty$ and acting on a symmetric function $F$ in the alphabet $X=x_{1}+x_{2}+x_{3}+\ldots$ according to the plethystic formulas

$$
\begin{aligned}
& D_{k} F[X]=\left.\left(F\left[X+\frac{M}{z}\right] \sum_{m \geq 0}(-z)^{m} e_{m}[X]\right)\right|_{z^{k}}, \\
& D_{k}^{*} F[X]=\left.\left(F\left[X-\frac{\tilde{M}}{z}\right] \sum_{m \geq 0} z^{m} h_{m}[X]\right)\right|_{z^{k}} .
\end{aligned}
$$

Here " $\left.\right|_{z^{k}}$ " denotes the operation of taking the coefficient of $z^{k}$ in the preceding expression, $e_{m}$ and $h_{m}$ denote the elementary and homogeneous symmetric functions 
indexed by $m$, and for convenience we have set

$$
M=(1-t)(1-q), \quad \widetilde{M}=(1-1 / t)(1-1 / q)
$$

These operators are connected to $\nabla$ and the polynomials $\tilde{H}_{\mu}$ through the following basic identities:
(i) $\quad D_{0} \tilde{H}_{\mu}=-D_{\mu}(q, t) \tilde{H}_{\mu}$
$(i)^{*} \quad D_{0}^{*} \tilde{H}_{\mu}=-D_{\mu}(1 / q, 1 / t) \tilde{H}_{\mu}$
$D_{k} \underline{e}_{1}-\underline{e}_{1} D_{k}=M D_{k+1}$
$\nabla \underline{e}_{1} \nabla^{-1}=-D_{1}$
$(i i)^{*} \quad D_{k}^{*} \underline{e}_{1}-\underline{e}_{1} D_{k}^{*}=-\widetilde{M} D_{k+1}^{*}$
(iv)

$$
\nabla^{-1} \partial_{1} \nabla=\frac{1}{M} D_{-1}
$$
$(i v)^{*}$
$\nabla D_{1}^{*} \nabla^{-1}=\underline{e}_{1}$

$$
\nabla^{-1} D_{-1}^{*} \nabla=-\widetilde{M} \partial_{1}
$$

where $\underline{e}_{1}$ is simply the operator "multiplication by $e_{1}$ " and $\partial_{1}$ is its "Hall" scalar product adjoint.

A close study of the operators $D_{k}, D_{k}^{*}$ led to the discovery of a number of remarkable symmetric function bases. The typical result here can be stated as follows. set

Theorem I.1. For $\lambda=\left(\lambda_{1}, \lambda_{2}, \ldots, \lambda_{s}, 1^{a}\right)$ with $\lambda_{1} \geq \lambda_{2} \geq \cdots \lambda_{s} \geq 2$ and $a \geq 0$

$$
W_{\lambda}[X ; q, t]=\underline{e}_{1}^{a} D_{1}^{*} \underline{e}_{1}^{\lambda_{1}-1} D_{1}^{*} \underline{e}_{1}^{\lambda_{2}-1} \cdots D_{1}^{*} \underline{e}_{1}^{\lambda_{s}-1}
$$

This given, the collection

$$
\left\{W_{\lambda}[X ; q, t]\right\}_{\lambda \vdash k}
$$

is a basis for the homogeneous symmetric polynomials of degree $k$. More precisely we have the expansion

$$
\left.P_{W(\lambda)}[X ; q, t]=\widetilde{M}^{r} h_{\lambda}+<\cdots \quad \text { (for some } r \leq k-1\right)
$$

where tihe symbol " $<\ldots$ " is to express that the remaining terms involve homogeneous basis elements which follow $h_{\lambda}$ in a suitable total order.

Of course the same identities hold true with $D_{1}^{*}$ replaced by $D_{1}$ in $I .13$.

At this point it is convenient to introduce, for a given quantity $Q$, the "translation" operator $\mathcal{T}_{Q}$ which acts on a symmetric function $P[X]$ according to the plethystic formula

$$
\mathcal{T}_{Q} P[X]=P[X+Q]
$$

This given, perhaps the most remarkable property of $\nabla$ is expressed by the following result, proved in [13]. 
TheOREM I.2. Let

$$
\Pi=\nabla^{-1} \mathcal{T}_{\epsilon}^{-1}
$$

and for a given symmetric function $F$ set

$$
\Pi_{F}=\Pi F=\nabla^{-1} F\left[X-^{-} 1\right] .
$$

Then we have

$$
\Pi_{F}\left[D_{\mu}(q, t)\right]=\left\langle F, \tilde{H}_{\mu}[X+1 ; q, t]\right\rangle_{*},
$$

where $\langle,\rangle_{*}$ is the scalar product defined by setting

$$
\left\langle\tilde{H}_{\lambda}, \tilde{H}_{\mu}\right\rangle_{*}= \begin{cases}\tilde{h}_{\mu} \tilde{h}_{\mu}^{\prime} & \text { if } \lambda=\mu \\ 0 & \text { otherwise }\end{cases}
$$

with

$$
\tilde{h}_{\mu}(q, t)=\prod_{s \in \mu}\left(q^{a_{\mu}(s)}-t^{l_{\mu}(s)+1}\right) \quad \text { and } \quad \tilde{h}_{\mu}^{\prime}(q, t)=\prod_{s \in \mu}\left(t^{l_{\mu}(s)}-q^{a_{\mu}(s)+1}\right) .
$$

We should point out that, in particular, this implies that certain images of $\nabla$ have rather surprising vanishing properties. More precisely, it follows immediately from $1.17^{(\dagger)}$ that

Corollary I.1. For any given symmetric function $F$ of degree $k$ we have

$$
\Pi_{F}\left[D_{\mu}(q, t)\right]= \begin{cases}0 & \text { if }|\mu|<k \\ \left\langle e_{n-k}^{*} F, \tilde{H}_{\mu}\right\rangle_{*} & \text { if }|\mu|=n \geq k\end{cases}
$$

where for convenience for any symmetric function $F[X]$ we set

$$
F^{*}[X]=F\left[\frac{X}{(1-t)(1-q)}\right] \text {. }
$$

This paper is divided into five sections. In the first section we state the basic identities we need in our developments, prove Theorem I.1 and derive from it the integrality of $\nabla$. We also derive there a number of other consequences of the identity in I.17. In the second section we work with the special case $t=1$ and relate the action of $\nabla_{t=1}$ to $q$-Lagrange inversion. In the third section we state a few positivity conjectures and establish a number of results in their support. In the fourth section, as a by-product of the symmetric function identities developed here and in [13] we derive a new formula for the $q, t$-Catalan introduced in [11] which makes it quite evident that it is a polynomial in $q$ and $t$. In the final section we prove a number of identities and derive a plethystic form of the higher indexed Macdonald operators.

1. Basic properties of $\nabla$. Let us recall that the Hall scalar product " $\langle$,$\rangle " on$

(†) See [13] Theorem I.2 
$\Lambda$ is deffined by setting for the power basis $\left\{p_{\rho}\right\}_{\rho}$

$$
\left\langle p_{\rho^{(1)}}, p_{\rho^{(2)}}\right\rangle= \begin{cases}z_{\rho} & \text { if } \rho^{(1)}=\rho^{(2)}=\rho \\ 0 & \text { otherwise }\end{cases}
$$

where for a partition $\rho=\left(1^{\alpha_{1}}, 2^{\alpha_{2}}, 3^{\alpha_{3}}, \cdots\right)$ we set as customary

$$
z_{\rho}=1^{\alpha_{1}} 2^{\alpha_{2}} 3^{\alpha_{3}} \cdots \alpha_{1} ! \alpha_{2} ! \alpha_{3} ! \cdots .
$$

We shall systematically use the symbol $\Omega[X]$ here as in [13] to represent the symmetric function

$$
\Omega[X]=\prod_{i} \frac{1}{1-x_{i}}=\exp \left(\sum_{k \geq 1} \frac{p_{k}}{k}\right) .
$$

In the same vein we shall also set

$$
\tilde{\Omega}[X]=\omega \Omega[X]=\prod_{i}\left(1+x_{i}\right)=\exp \left(\sum_{k \geq 1} \frac{(-1)^{k-1} p_{k}}{k}\right) .
$$

This given, we have the following basic expansions ([13] Theorem 1.3):

$$
\begin{aligned}
& \text { a) } \tilde{\Omega}\left[\frac{X Y}{(1-q)(1-t)}\right]=\sum_{\rho} \frac{p_{\rho}[X] p_{\rho}[Y]}{(-1)^{|\rho|-l(\rho)} z_{\rho} p_{\rho}[(1-t)(1-q)]} \\
& \text { b) } \tilde{\Omega}\left[\frac{X Y}{(1-q)(1-t)}\right]=\sum_{\lambda} S_{\lambda}\left[\frac{X}{(1-q)(1-t)}\right] S_{\lambda^{\prime}}[Y]=\sum_{\lambda} S_{\lambda}^{*}[X] S_{\lambda^{\prime}}[Y] \\
& \text { c) } \tilde{\Omega}\left[\frac{X Y}{(1-q)(1-t)}\right]=\sum_{\mu} \frac{\tilde{H}_{\mu}[X ; q, t] \tilde{H}_{\mu}[Y ; q, t]}{\tilde{h}_{\mu}(q, t) \tilde{h}_{\mu}^{\prime}(q, t)}
\end{aligned}
$$

In particular we see from $1.2 \mathrm{c})$ that $\tilde{\Omega}\left[\frac{X Y}{(1-q)(1-t)}\right]$ is the reproducing kernel of the *-scalar product defined by I.18. We also see from 1.2 a) that the $*$-scalar product may also be defined by setting

$$
\left\langle p_{\rho^{(1)}}, p_{\rho^{(2)}}\right\rangle_{*}= \begin{cases}(-1)^{|\rho|-l(\rho)} z_{\rho} p_{\rho}[(1-t)(1-q)] & \text { if } \rho^{(1)}=\rho^{(2)}=\rho, \\ 0 & \text { otherwise. }\end{cases}
$$

From this we derive that the Hall and the $*$-scalar products are simply related by the identity

$$
\langle P, Q\rangle_{*}=\langle P, \omega \phi Q\rangle
$$

where for convenience we set, for every $F[X] \in \Lambda$,

$$
\phi F[X]=F[X(1-t)(1-q)]=F[X M] .
$$

Here and after we shall denote by " $\underline{S}_{\mu}$ " the operator "multiplication", by the Schur function $S_{\mu}$. Note that since the skew schur function $S_{\lambda / \mu}$ is defined from the expan- 
sion

$$
S_{\lambda / \mu}=\sum_{\nu} S_{\nu}\left\langle S_{\mu} S_{\nu}, S_{\lambda}\right\rangle
$$

we see that the Hall scalar product adjoint of $\underline{S}_{\mu}$ is the operator " $\partial_{S_{\mu}}$ " defined by setting for the Schur function basis

$$
\partial_{S_{\mu}} S_{\lambda}=S_{\lambda / \mu}
$$

We should point out that we have also set

$$
\partial_{S_{1}}=\delta_{1}
$$

We shall also need some more general "translation" and "multiplication" operators defined by setting set for every $Q \in \Lambda$ and any alphabet $Y$

$$
\begin{aligned}
& \text { a) } \mathcal{T}_{Y} Q[X]=Q[X+Y] \\
& \text { b) } \quad \mathcal{P}_{Y} Q[X]=\Omega[X Y] Q[X] .
\end{aligned}
$$

It is easy to show (see [13]) that these operators have the following useful "Schur function" expansions:

$$
\begin{aligned}
& \text { a) } \mathcal{T}_{Y}=\sum_{\mu} S_{\mu}[Y] \partial_{S_{\mu}}, \\
& \text { b) } \quad \mathcal{P}_{Y}=\sum_{\mu} S_{\mu}[Y] \underline{S}_{\mu},
\end{aligned}
$$

which show that $\mathcal{P}_{Y}$ is the Hall adjoint of $\mathcal{T}_{Y}$. Note that if $X$ consists of a single variable $u$ we have

$$
\mathcal{T}_{u}=\sum_{m \geq 0} u^{m} \partial_{S_{m}} .
$$

It is also important to note that we have the commutativity relation (see [13])

$$
\mathcal{T}_{Y} \mathcal{P}_{Z}=\Omega[Y Z] \mathcal{P}_{Z} \mathcal{T}_{Y}
$$

Another useful ingredient which occurs in our developments is the involution " $\downarrow$ " defined by setting for any $F \in \Lambda_{\mathbf{Z}[q, t, 1 / q, 1 / t]}$

$$
\downarrow F[X ; q, t]=\omega F[X ; 1 / q, 1 / t]
$$

It can be shown (see [12]) that we have

$$
\downarrow \tilde{H}_{\mu}[X ; q, t]=\frac{1}{T_{\mu}} \tilde{H}_{\mu}[X ; q, t] .
$$

We also have

$$
\begin{array}{ll}
\text { a) } & \downarrow \mathcal{T}_{1} \downarrow=\mathcal{T}_{\epsilon}^{-1} \\
\text { b) } & \downarrow \nabla \downarrow=\nabla^{-1} \\
\text { c) } & \downarrow D_{k} \downarrow=(-1)^{k} D_{k}^{*} .
\end{array}
$$


Finally, we should point out that by combining I.5, I.12 $(i)$ and $(i)^{*}$ with $\left.1.2 \mathrm{c}\right)$ we deduce that the operators $\nabla, D_{0}$ and $D_{0}^{*}$ are all self-adjoint with respect to the *scalar product.

This completes the collection of basic facts we shall need in our further developments.

Proof of Theorem I.1. To show that the polynomials

$$
W_{\lambda}[X ; q, t]=\underline{e}_{1}^{a} D_{1}^{*} \underline{e}_{1}^{\lambda_{1}-1} D_{1}^{*} \underline{e}_{1}^{\lambda_{2}-1} \cdots D_{1}^{*} \underline{e}_{1}^{\lambda_{s}-1} 1 \quad \lambda_{1} \geq \lambda_{2} \geq \cdots \geq \lambda_{s}>1
$$

form a basis of $\Lambda$, it is sufficient to establish that those for which $a=0$ and $\lambda_{1}+$ $\lambda_{2}+\cdots+\lambda_{s}=k$, span $\Lambda^{=k}$ modulo $\underline{e}_{1} \Lambda^{=k-1}$. To this end note that for any $m>1$ and $F \in \Lambda$ we have

$$
\begin{aligned}
D_{1}^{*} e_{1}^{m-1} F & =\left.\left(e_{1}-\widetilde{M} / z\right)^{m-1} F[X-\widetilde{M} / z] \Omega[z X]\right|_{z} \\
& \left.\equiv(-\widetilde{M} / z)^{m-1} F[X-\widetilde{M} / z] \Omega[z X]\right|_{z} \quad\left(\bmod \underline{e}_{1} \Lambda\right) \\
& =\left.\left.(-\widetilde{M})^{m-1} \sum_{k \geq 0} F[X-\widetilde{M} / z]\right|_{1 / z^{k}} \Omega[z X]\right|_{z^{m+k}} \\
& =(-\widetilde{M})^{m-1} h_{m}[X] F[X]+\left(\text { terms in } h_{m+1}, h_{m+2}, \ldots\right) .
\end{aligned}
$$

Thus it follows that, when all $\lambda_{i}>1$,

$W_{\lambda}[X ; q, t]=D_{1}^{*} e_{1}^{\lambda_{1}-1} D_{1}^{*} e_{1}^{\lambda_{2}-1} \cdots D_{1}^{*} e_{1}^{\lambda_{s}-1}=(-\widetilde{M})^{|\lambda|-l(\lambda)} h_{\lambda_{1}} h_{\lambda_{2}} \cdots h_{\lambda_{s}}+\cdots$

where the omitted terms involve complete homogeneous basis elements $h_{\mu}$ with $\mu>\lambda$ in lexicographic order. This shows that the collection

$$
\mathcal{B}^{=k}=\left\{W_{\lambda}[X ; q, t]: \lambda \vdash k \& \text { all } \lambda_{i}>1\right\}
$$

is an independent set in $\Lambda^{=k} / \underline{e}_{1} \Lambda^{j=k-1}$. To complete our proof we need only verify that the cardinality of $\mathcal{B}^{=k}$ is equal to the dimension of $\Lambda^{=k} / \underline{e}_{1} \Lambda^{=k-1}$. In other words we must show that

$$
\#\left\{\lambda: \lambda \vdash k \& \lambda_{i}>1\right\}=\#\{\lambda: \lambda \vdash k\}-\#\{\lambda: \lambda \vdash k-1\} .
$$

However, this follows immediately by equating coefficients of $q^{k}$ in the power series identity

$$
\prod_{m \geq 2} \frac{1}{1-q^{m}}=(1-q) \prod_{m \geq 1} \frac{1}{1-q^{m}} \text {. }
$$

REMARK 1.1. Note that, since the definition in 1.12 gives that $\downarrow 1=1$ and $\downarrow \underline{e}_{1} \downarrow==\underline{e}_{1}$, from $1.14 \mathrm{c}$ ) we derive that

$$
\downarrow W_{\lambda}[X ; q, t]=(-1)^{s} \underline{e}_{1}^{a} D_{1} \underline{e}_{1}^{\lambda_{1}-1} D_{1} \underline{e}_{1}^{\lambda_{2}-1} \cdots D_{1} \underline{e}_{1}^{\lambda_{s}-1} 1 .
$$

Thus, the invertibility of " $\downarrow$ " yields that also these polynomials form a basis of $\Lambda$. In fact, more than that is true. For a partition $\lambda=\left(\lambda_{1} \geq \lambda_{2} \geq \cdots \geq \lambda_{s}>1^{a}\right)$ set

$$
U_{\lambda}[X ; q, t]=D_{1}^{a} \underline{e}_{1} D_{1}^{\lambda_{1}-1} \underline{e}_{1} D_{1}^{\lambda_{2}-1} \cdots \underline{e}_{1} D_{1}^{\lambda_{s}-1} 1 .
$$

This given we have 
ThEOREM 1.1. The operator $\nabla$ may be computed from the identity

$$
\nabla W_{\lambda}[X ; q, t]=(-1)^{a+\sum_{i=1}^{s}\left(\lambda_{i}-1\right)} U_{\lambda}[X ; q, t] .
$$

In particular, the collection

$$
\left\{U_{\lambda}[X ; q, t]\right\}_{\lambda}
$$

is also a basis, and necessarily $\nabla$ is a polynomial operator, that is

$$
\nabla \Lambda_{Z[q, t]} \subseteq \Lambda_{Z[q, t]}
$$

Proof. Note that from I.12 (iii) and $(i i i)^{*}$ written in the form
a) $\nabla \underline{e}_{1}=-D_{1} \nabla$
b) $\nabla D e_{1}^{*}=\underline{e}_{1} \nabla$

it follows that

$$
\nabla W_{\lambda}[X ; q, t]=\left(-D_{1}\right)^{a} \underline{e}_{1}\left(-D_{1}\right)^{\lambda_{1}-1} \underline{e}_{1}\left(-D_{1}\right)^{\lambda_{2}-1} \cdots \underline{e}_{1}\left(-D_{1}\right)^{\lambda_{s}-1} 1,
$$

and this is 1.18. The fact that the collection in 1.19 is a basis then follows from Theorem I.1 and the invertibility of $\nabla$. Note next that the triangularity expressed by 1.15 , together with a closer look at the later terms, yields that, at the very worst, the complete homogeneous basis $\left\{h_{\lambda}[X]\right\}_{\lambda}$ admits an expansion in terms of the basis $\left\{W_{\lambda}[X ; q, t]\right\}_{\lambda}$ with coefficients in $Z\left[q, t, 1 / q, 1 / t, M^{-1}\right]$. Combining this with 1.18 and the definition of $D_{1}$ yields that

$$
\nabla \Lambda_{Z} \subseteq Z\left[q, t, 1 / q, 1 / t, M^{-1}\right] \Lambda_{Z}
$$

In other words, at worst, $\nabla$ introduces powers of $t, q,(1-t)$ and $(1-q)$, in the denominators. To eliminate these denominators we must show that for $F \in \Lambda_{Z}, \nabla F$ has no pole at $q=0, t=0, q=1$ and $t=1$. By symmetry, it is sufficient to deal with the cases $q=0,1$, and for these we can take $F$ to be an element of any basis we choose of $Z(t) \Lambda_{Z}$.

For $q=0$ we may take $F=\tilde{H}_{\mu}[X ; 0, t]$. In fact, it follows from I.7 [22, ch VI (8.4) (ii)] that

$$
\tilde{H}_{\mu}[X ; 0, t]=\sum_{\lambda} S_{\lambda}[X] \tilde{K}_{\lambda \mu}(t)
$$

with $\tilde{K}_{\lambda \mu}(t)$ the Kostka-Foulkes polynomials. We may write 1.21 , for a particular degree $d$, in matrix form as

$$
\langle\tilde{H}[X ; 0, t]\rangle_{d}=\langle S[X]\rangle_{d} \tilde{K}_{d}(t) .
$$

In the same vein we may write 1.7 in the form

$$
\langle\tilde{H}[X ; q, t]\rangle_{d}=\langle S[X]\rangle_{d} \tilde{K}_{d}(q, t) .
$$

Now 1.22 and 1.23 may be combined into

$$
\langle\tilde{H}[X ; q, t]\rangle_{d}=\langle\tilde{H}[X ; 0, t]\rangle_{d} \tilde{K}(t)_{d}^{-1} \tilde{K}_{d}(q, t) .
$$

Thus the polynomiality of the $\tilde{K}_{\lambda \mu}(q, t)([14]$, [15]) yields that the entries of the matrix

$$
\left\|a_{\lambda \mu}(q, t)\right\|_{\lambda, \mu \vdash d}=\tilde{K}(t)_{d}^{-1} \tilde{K}_{d}(q, t)
$$


are necessarily polynomials in $q$ with coefficients rational functions of $t$; in particular they have no poles at $q=0$. Since $\left\|a_{\lambda \mu}(q, t)\right\|_{\lambda, \mu \vdash d}$ tends to the identity matrix as $q \rightarrow 0$, its determinant does not vanish at $q=0$. This implies that, by inverting $\left\|a_{\lambda \mu}(q, t)\right\|_{\lambda, \mu \vdash d}$, we will obtain an expansion

$$
\tilde{H}_{\lambda}[X ; 0, t]=\sum_{\mu} b_{\lambda \mu}(q, t) \tilde{H}_{\mu}[X ; q, t],
$$

with the $b_{\lambda \mu}(q, t)$ rational functions in $q$ and $t$ with no poles at $q=0$. Now I.5 gives

$$
\nabla \tilde{H}_{\lambda}[X ; 0, t]=\sum_{\mu} b_{\lambda \mu}(q, t) t^{n(\mu)} q^{n\left(\mu^{\prime}\right)} \tilde{H}_{\mu}[X ; q, t] .
$$

Since nothing on the right hand side has a pole at $q=0$, this completes our argument for the case $q=0$.

We can treat the case $q=1$ in an entirely analogous manner using the basis $\left\{\tilde{H}_{\mu}[X ; 1, t]\right\}_{\mu}$. In fact, it follows from [22 ch VI 8.4 (iii)] that

$$
\tilde{H}_{\mu}[X ; 1, t]=\prod_{i=1}^{l\left(\mu^{\prime}\right)}(t ; t)_{\mu_{i}^{\prime}} h_{\mu_{i}^{\prime}}\left[\frac{X}{1-t}\right] .
$$

Now it is well known that for any integer $m \geq 1$ we have the Schur function expansion

$$
(t ; t)_{m} h_{m}\left[\frac{X}{1-t}\right]=\sum_{\lambda} S_{\lambda}[X] \sum_{T \in S T(\lambda)} t^{c o(T)} .
$$

where the inner sum is over all standard tableaux of shape $\lambda$ and " $c o(T)$ " denotes cocharge. Thus using 1.26 in 1.25 we obtain the Schur function expansion

$$
\tilde{H}_{\mu}[X ; 1, t]=\sum_{\lambda} S_{\lambda}[X] \theta_{\lambda \mu}(t)
$$

with coefficients $\theta_{\lambda \mu}(t)$ polynomials with positive integer coefficients. Writing this in mat:cix form as we did before gives

$$
\langle\tilde{H}[X ; 1, t]\rangle_{d}=\langle S[X]\rangle_{d} \theta_{d}(t) .
$$

So ve may combine it with 1.23 and obtain

$$
\langle\tilde{H}[X ; q, t]\rangle_{d}=\langle\tilde{H}[X ; 1, t]\rangle_{d} \theta_{d}(t)^{-1} \tilde{K}_{d}(q, t),
$$

and the argument proceeds precisely in the same manner as before since the matrix $\theta_{d}(t)^{-1} \tilde{K}_{d}(q, t)$ again approaches the identity as $q \rightarrow 1$. This completes our proof.

CoROLLARY 1.1. The operator $\nabla^{-1}$ is a Laurent polynomial operator, i.e. $\nabla^{-1} \Lambda_{\mathbf{Z}}$ $\subseteq \mathbf{Z}[q, t, 1 / q, 1 / t] \Lambda_{\mathbf{Z}}$

Proof. Theorem I.1 guarantees that the matrix of $\nabla$ with respect to any basis of $\Lambda \overline{\mathbf{Z}}^{d}$ has entries in $\mathbf{Z}[q, t]$. Since the eigenvalues of $\nabla$ on $\Lambda_{\overline{\mathbf{Z}}}^{d}$ are $t^{n(\mu)} q^{n\left(\mu^{\prime}\right)}$ with $|\mu|=d$, the determinant of all these $\nabla$ matrices consists of a monomial in $q$ and $t$. Thus all their inverses have entries in $\mathbf{Z}[q, t, 1 / q, 1 / t]$.

COROLlaRY 1.2. The image of a Schur function under $\nabla$ has a Schur function expansion with coefficients in $\mathbf{Z}[q, t]$. In particular formula (15) of [11], conjectured to give the Frobenius characteristic of diagonal harmonics, is a polynomial in $q$ and $t$. 
Proof. The first assertion is a particular case of Theorem I.1. The second assertion follows from (15) of [11] which essentially states that this Frobenius characteristic is $\nabla e_{n}[X]$.

There is an interesting family of operators which are closely related to $\nabla$ and have similar properties. More precisely, for any symmetric function $F \in \Lambda_{\mathbf{Z}[q, t]}$ we let $\Delta_{F}$ be the operator defined by setting on the $\left\{\tilde{H}_{\mu}\right\}_{\mu}$ basis

$$
\Delta_{F} \tilde{H}_{\mu}=F\left[B_{\mu}\right] \tilde{H}_{\mu}
$$

It is easily seen, from the definition of plethystic substitutions, that when $|\mu|=n$, $\nabla$ itself may be viewed as $\Delta_{e_{n}}$. Most interesting special cases of these operators are obtained by setting $F=e_{k}$. For instance we see from I.4 and I.12 $(i)$ that $D_{0}=-M \Delta_{e_{1}}+I$. Moreover, we have the following basic extensions of the table in I.12.

THEOREM 1.2.

$$
\Delta_{e_{1}} \underline{e}_{1}=\underline{e}_{1} \Delta_{e_{1}}-D_{1}
$$

$$
\Delta_{e_{1}} D_{1}^{*}=D_{1}^{*} \Delta_{e_{1}}+\underline{e}_{1}
$$

$$
\Delta_{e_{k}} \underline{e}_{1}=\underline{e}_{1} \Delta_{e_{k}}-D_{1} \Delta_{e_{k-1}}
$$$$
(v i)^{*} \quad \Delta_{e_{k}} D_{1}^{*}=D_{1}^{*} \Delta_{e_{k}}+\underline{e}_{1} \Delta_{e_{k-1}}
$$

Proof. It will be convenient to set, for a variable $u$ :

$$
\Psi(u) \tilde{H}_{\mu}=\sum_{k=0}^{|\mu|} u^{k} \Delta_{e_{k}} \tilde{H}_{\mu}=\left(\prod_{s \in \mu}\left(1+u t^{l_{\mu}^{\prime}(s)} q^{a_{\mu}^{\prime}(s)}\right)\right) \tilde{H}_{\mu} .
$$

It follows from the Macdonald Pieri rules [22] that for any partition $\nu$ we have the expansion

$$
\underline{e}_{1} \tilde{H}_{\nu}=\sum_{\mu \leftarrow \nu} \tilde{H}_{\mu} d_{\mu \nu}(q, t)
$$

where the coefficients $d_{\lambda \mu}(q, t)$ are rational functions in $q$ and $t$ which may be explicitly computed (see [9]). Their true nature is immaterial for us here. The important element is that the sum in 1.31 is carried out only over the partitions $\mu$ that immediately follow $\nu$ in the containment order. This given, we immediately derive from 1.30 that we have

$$
\Psi(u) \underline{e}_{1} \Psi(u)^{-1} \tilde{H}_{\nu}=\sum_{\mu \leftarrow \nu}\left(1+u w_{\mu \nu}\right) \tilde{H}_{\mu} d_{\mu \nu}(q, t)
$$

where $w_{\mu \nu}$ is the weight of the cell we must add to $\nu$ to get $\mu$. Now from the definition in I.4, we see that we must have $w_{\mu \nu}=T_{\mu} / T_{\nu}$ Thus 1.32 may be rewritten in following equivalent forms

$$
\begin{aligned}
\Psi(u) \underline{e}_{1} \Psi(u)^{-1} \tilde{H}_{\nu} & =\sum_{\mu \leftarrow \nu}\left(1+u T_{\mu} / T_{\nu}\right) \tilde{H}_{\mu} d_{\mu \nu}(q, t) \\
& =\sum_{\mu \leftarrow \nu} \tilde{H}_{\mu} d_{\mu \nu}(q, t)+u \nabla \sum_{\mu \leftarrow \nu} \tilde{H}_{\mu} d_{\mu \nu}(q, t) / T_{\nu} \\
& =\underline{e}_{1} \tilde{H}_{\mu}+u \nabla \underline{e}_{1} \tilde{H}_{\nu} / T_{\nu} \\
& =\underline{e}_{1} \tilde{H}_{\mu}+u \nabla \underline{e}_{1} \nabla^{-1} \tilde{H}_{\nu}=\underline{e}_{1} \tilde{H}_{\nu}-u D_{1} \tilde{H}_{\nu}
\end{aligned}
$$


where the last equality is due to I.12 (iii). Since $\nu$ is arbitrary we must conclude that

$$
\Psi(u) \underline{e}_{1} \Psi(u)^{-1}=\underline{e}_{1}-u D_{1} .
$$

Better yet we must also have

$$
\Psi(u) \underline{e}_{1}=\underline{e}_{1} \Psi(u)-u D_{1} \Psi(u) .
$$

In particular, equating coefficients of $u^{k}$ for $k \geq 1$ gives

$$
\text { 1) } \Delta_{e_{1}} \underline{e}_{1}=\underline{e}_{1} \Delta_{e_{1}}-D_{1}, \quad \text { k) } \Delta_{e_{k}} \underline{e}_{1}=\underline{e}_{1} \Delta_{e_{k}}-D_{1} \nabla_{e_{k-1}} \text {. }
$$

This proves $1.29(v)$ and $1.29(v i)$. Now, using I.12 (iii)* we may eliminate $\underline{e}_{1}$ from these equations and obtain
1) $\Delta_{e_{1}} \nabla D_{1}^{*} \nabla^{-1}=\nabla D_{1}^{*} \nabla^{-1} \Delta_{e_{1}}-D_{1}$,
k) $\Delta_{e_{k}} \nabla D_{1}^{*} \nabla^{-1}=\nabla D_{1}^{*} \nabla^{-1} \Delta_{e_{k}}-D_{1} \nabla_{e_{k-1}}$.

Since $\nabla^{\prime}$ necessarily commutes with any of the operators $\Delta_{F}$, these two relations may also be rewritten in the form

1) $\left.\Delta_{e_{1}} D_{1}^{*}=D_{1}^{*} \Delta_{e_{1}}-\nabla^{-1} D_{1} \nabla, \quad k\right) \Delta_{e_{k}} D_{1}^{*}=D_{1}^{*} \Delta_{e_{k}}-\nabla^{-1} D_{1} \nabla \nabla_{e_{k-1}}$,

and a vise of I.12 (iii) finally simplifies them to $1.29(v)^{*}$ and $1.29(v i)^{*}$, completing our proof.

It develops that the argument that yielded the integrality of $\nabla$ can be applied also to the operators $\Delta_{F}$. In fact, the identities in 1.29 are precisely what is needed to compute the action of any of the operators $\Delta_{F}$ without resorting to expansions in terms of the basis $\left\{\tilde{H}_{\mu}\right\}_{\mu}$.

THEOREM 1.3. For each $F \in \Lambda_{\mathbf{Z}[q, t]}$ we have

$$
\Delta_{F} \Lambda_{\mathbf{Z}} \subseteq \mathbf{Z}[q, t] \Lambda_{\mathbf{Z}}
$$

Proof. Note first that since the map $F \rightarrow \Delta_{F}$ is linear and multiplicative in $F$ it is sufficient to prove 1.35 for all the factors occurring in an integral multiplicative basis of $\Lambda_{\mathbf{Z}[q, t]}$. In particular, we may choose the "elementary" symmetric function basis. This reduces us to verify 1.35 when $F=e_{k}$. We have seen in the proof of Theorem 1.1 , that the expansion of the complete homogeneous basis $\left\{h_{\lambda}\right\}_{\lambda}$ in terms of the basis $\left\{W_{\lambda}[X ; q, t]\right\}_{\lambda}$ has coefficients whose denominators only contain powers of $q, t, 1-q$ and $1-t$. To follow the same strategy we used in the proof of Theorem 1.1, the first part of our proof will be to show that the action of any of the operators $\Delta_{e_{k}}$ on the basis $\left\{W_{\lambda}[X ; q, t]\right\}_{\lambda}$ introduces no other kinds of denominator factors. To this end, note first that for $a \geq 1$ in I.13, using 1.29 (vi) we get

$$
\begin{aligned}
\Delta_{e_{k}} W_{\lambda}[X ; q, t]=\underline{e}_{1} \Delta_{e_{k}} \underline{e}_{1}{ }^{a-1} D_{1}^{*} \underline{e}_{1}^{\lambda_{1}-1} D_{1}^{*} \underline{e}_{1}^{\lambda_{2}-1} \cdots D_{1}^{*} \underline{e}_{1}^{\lambda_{s}-1} \\
\\
-D_{1} \Delta_{e_{k-1} \underline{e}_{1}}{ }^{a-1} D_{1}^{*} \underline{e}_{1}^{\lambda_{1}-1} D_{1}^{*} \underline{e}_{1}^{\lambda_{2}-1} \cdots D_{1}^{*} \underline{e}_{1}^{\lambda_{s}-1} .
\end{aligned}
$$

On the other hand, if $a=0$ then $1.29(v i)^{*}$ gives

$$
\begin{aligned}
\Delta_{e_{k}} W_{\lambda}[X ; q, t]=D_{1}^{*} \Delta_{e_{k}} \underline{e}_{1}^{\lambda_{1}-1} D_{1}^{*} \underline{e}_{1}^{\lambda_{2}-1} \cdots D_{1}^{*} \underline{e}_{1}^{\lambda_{s}-1} \\
+\underline{e}_{1} \Delta_{e_{k-1}} \underline{e}_{1}^{\lambda_{1}-1} D_{1}^{*} \underline{e}_{1}^{\lambda_{2}-1} \cdots D_{1}^{*} \underline{e}_{1}^{\lambda_{s}-1} .
\end{aligned}
$$

From these two relations we immediately see that, by means of the relations in 1.29 and a double induction argument based on $k$ and the size of $\lambda$, we can establish that 
$\Delta_{e_{k}} W_{\lambda}[X ; q, t]$ always results in a Z-linear combination of polynomials of the form

$$
O_{1} O_{2} \cdots O_{m} 1 \quad \text { with each } \quad O_{i}=\underline{e}_{1}, D_{1} \text { or } D_{1}^{*} .
$$

This completes the first part of the proof since $\underline{e}_{1}$ and $D_{1}$ are clearly integral (see I.10) and $D_{1}^{*}$ at worst introduces only denominator factors which are powers of $q t$.

We are now in the same position as before to show that the image by $\Delta_{e_{k}}$ of any $h_{\lambda}$ contains no poles at $q=0, t=0, q=1$ and $t=1$. In fact, for the case $q=0$, for instance, we may use 1.24 again and derive that

$$
\Delta_{e_{k}} \tilde{H}_{\lambda}[X ; 0, t]=\sum_{\mu} b_{\lambda \mu}(q, t) e_{k}\left[B_{\mu}(q, t)\right] \tilde{H}_{\mu}[X ; q, t]
$$

which shows that the action of $\Delta_{e_{k}}$ on the basis $\left\{\tilde{H}_{\lambda}[X ; 0, t]\right\}_{\lambda}$ produces no poles at $q=0$. Similar arguments eliminate poles at $t=0, q=1$ and $t=1$, completing the proof of 1.36 .

Our next and final task in this section is to derive some interesting applications of Theorem I.2. We shall see that formula I.17 with $\Pi$ given by $\mathrm{I} .16$ encodes a remarkable amount of combinatorial information. To this end we recall that the coefficients $c_{\mu \nu}(q, t)$ which occur in the identity

$$
\delta_{1} \tilde{H}_{\mu}[X ; q, t]=\sum_{\nu \rightarrow \mu} c_{\mu \nu}(q, t) \tilde{H}_{\nu}[X ; q, t]
$$

have the following important property.

Proposition 1.1.

$$
\sum_{\nu \rightarrow \mu} c_{\mu \nu}(q, t)\left(T_{\mu} / T_{\nu}\right)^{k}= \begin{cases}\frac{t q}{M} h_{k+1}\left[D_{\mu}(q, t) / t q\right] & \text { if } k \geq 1 \\ B_{\mu}(q, t) & \text { if } k=0 .\end{cases}
$$

We should mention that it follows from the Macdonald Pieri rules [9] that for $\nu \rightarrow \mu$ we have

$$
c_{\mu \nu}(q, t)=\prod_{s \in R_{\mu \nu}} \frac{t^{l_{\mu}(s)}-q^{a_{\mu}(s)+1}}{t^{l_{\nu}(s)}-q^{a_{\nu}(s)+1}} \prod_{s \in C_{\mu \nu}} \frac{q^{a_{\mu}(s)}-t^{l_{\mu}(s)+1}}{q^{a_{\nu}(s)}-t^{l_{\nu}(s)+1}},
$$

where $R_{\mu \nu}$ and $C_{\mu \nu}$ denote the collections of cells of $\nu$ that are respectively in the row and the column of the cell $\mu / \nu$. Similarly, the coefficients $d_{\mu \nu}(q, t)$ occurring in 1.31 are given by the formula

$$
d_{\mu \nu}(q, t)=\prod_{s \in R_{\mu \nu}} \frac{q^{a_{\nu}(s)}-t^{l_{\nu}(s)+1}}{q^{a_{\mu}(s)}-t^{l_{\mu}(s)+1}} \prod_{s \in C_{\mu \nu}} \frac{t^{l_{\nu}(s)}-q^{a_{\nu}(s)+1}}{t^{l_{\mu}(s)}-q^{a_{\mu}(s)+1}} .
$$

The identity in 1.37 was proved in [15] by taking full account of the combinatorial information contained in 1.38. An analogous argument may also be used to prove the following analogue of 1.37 . 
Proposition 1.2.

$$
\sum_{\mu \leftarrow \nu} d_{\mu \nu}(q, t)\left(T_{\mu} / T_{\nu}\right)^{k}= \begin{cases}(-1)^{k-1} e_{k-1}\left[D_{\nu}(q, t)\right] & \text { if } k \geq 1 \\ 1 & \text { if } k=0\end{cases}
$$

Now it; develops that both 1.37 and 1.40 can be derived with considerably less effort directly from I.17 without resorting to 1.38 or 1.39 . To illustrate this type of argument we shall carry out the derivation of 1.40 .

To begin with we should point out that 1.37 and 1.40 follow from two master identities that are interesting in themselves.

Theorem 1.4. For any $P \in \Lambda$ we have

$$
\begin{gathered}
\sum_{\nu \rightarrow \mu} c_{\mu \nu}(q, t) P\left[D_{\nu}(q, t)\right]=\frac{1}{M} P\left[D_{\mu}(q, t)\right]+\frac{1}{M}\left(D_{1}^{*} P\right)\left[D_{\mu}(q, t)\right] \\
\sum_{\mu \leftarrow \nu} d_{\mu \nu}(q, t) P\left[D_{\mu}(q, t)\right]=P\left[D_{\nu}(q, t)\right]+\left(D_{-1} P\right)\left[D_{\nu}(q, t)\right]
\end{gathered}
$$

Proof. For convenience let

$$
F=\Pi^{-1} P=\tau_{\epsilon} \nabla P .
$$

This given, we may write the left hand side of 1.41 in the following equivalent forms

$$
\begin{aligned}
\sum_{\nu \rightarrow \mu} c_{\mu \nu}(q, t) P\left[D_{\nu}(q, t)\right] & =\sum_{\nu \rightarrow \mu} c_{\mu \nu}(q, t) \Pi_{F}\left[D_{\nu}(q, t)\right] \\
(\text { by I.17) } & =\sum_{\nu \rightarrow \mu} c_{\mu \nu}(q, t)\left\langle F, \mathcal{T}_{1} \tilde{H}_{\nu}\right\rangle_{*} \\
(\text { by 1.36 }) & =\left\langle F, \mathcal{T}_{1} \delta_{1} \tilde{H}_{\mu}\right\rangle_{*} \\
(\text { by 1.10 }) & =\left\langle F, \delta_{1} \mathcal{T}_{1} \tilde{H}_{\mu}\right\rangle_{*} \\
(\text { by 1.4 }) & =\left\langle\phi \omega F, \delta_{1} \mathcal{T}_{1} \tilde{H}_{\mu}\right\rangle=\left\langle\underline{e}_{1} \phi \omega F, \mathcal{T}_{1} \tilde{H}_{\mu}\right\rangle \\
& =\frac{1}{M}\left\langle\underline{e}_{1} F, \mathcal{T}_{1} \tilde{H}_{\mu}\right\rangle_{*} .
\end{aligned}
$$

Thus one further application of $\mathrm{I} .17$ yields us

$$
\sum_{\nu \rightarrow \mu} c_{\mu \nu}(q, t) P\left[D_{\nu}(q, t)\right]=\frac{1}{M} \Pi_{e_{1} F}\left[D_{\mu}(q, t)\right] .
$$

However, using I.16 and 1.43 we get

$$
\Pi_{e_{1} F}=\nabla^{-1} \mathcal{T}_{\epsilon}^{-1} \underline{e}_{1} \mathcal{T}_{\epsilon} \nabla P=\nabla^{-1}\left(e_{1}+1\right) \nabla P .
$$

This enables us to rewrite 1.44 in the form

$$
\sum_{\nu \rightarrow \mu} c_{\mu \nu}(q, t) P\left[D_{\nu}(q, t)\right]=\frac{1}{M} P\left[D_{\mu}(q, t)\right]+\frac{1}{M}\left(\nabla^{-1} e_{1} \nabla P\right)\left[D_{\mu}(q, t)\right],
$$

which, in view of $1.12(i i i)^{*}$, is just another way of writing 1.41 . 
To prove 1.42 we can again start with 1.43 and get

$$
\begin{aligned}
\sum_{\mu \leftarrow \nu} d_{\mu \nu}(q, t) P\left[D_{\mu}(q, t)\right] & =\sum_{\mu \leftarrow \nu} d_{\mu \nu}(q, t) \Pi_{F}\left[D_{\mu}(q, t)\right] \\
(\text { by I.17 }) & =\sum_{\mu \leftarrow \nu} d_{\mu \nu}(q, t)\left\langle F, \mathcal{T}_{1} \tilde{H}_{\mu}\right\rangle_{*} \\
(\text { by } 1.31) & =\left\langle F, \mathcal{T}_{1} \underline{e}_{1} \tilde{H}_{\nu}\right\rangle_{*}=\left\langle F,\left(\underline{e}_{1}+1\right) \mathcal{T}_{1} \tilde{H}_{\nu}\right\rangle_{*} \\
& =M\left\langle\delta_{1} F, \mathcal{T}_{1} \tilde{H}_{\nu}\right\rangle_{*}+\left\langle F, \mathcal{T}_{1} \tilde{H}_{\nu}\right\rangle_{*},
\end{aligned}
$$

and another application of $\mathrm{I} .17$ gives

$$
\sum_{\mu \leftarrow \nu} d_{\mu \nu}(q, t) P\left[D_{\mu}(q, t)\right]=P\left[D_{\nu}(q, t)\right]+M \Pi_{\delta_{1} F}\left[D_{\nu}(q, t)\right] .
$$

Now, using 1.43 again we have

$$
\Pi_{\delta_{1} F}=\nabla^{-1} \mathcal{T}_{\epsilon}^{-1} \delta_{1} \mathcal{T}_{\epsilon} \nabla P=\nabla^{-1} \delta_{1} \nabla P
$$

and I.12 $(i v)$ transforms 1.45 into 1.42, completing the proof of the theorem.

Proof of Proposition 1.2. Note first that setting $P=1$ in 1.42 gives

$$
\sum_{\mu \leftarrow \nu} d_{\mu \nu}(q, t)=1
$$

as desired. Next, for $k \geq 1$, we choose $P$ to be the power symmetric function $p_{k}$ in 1.42 and get

$$
\sum_{\mu \leftarrow \nu} d_{\mu \nu}(q, t) D_{\mu}\left(q^{k}, t^{k}\right)=D_{\nu}\left(q^{k}, t^{k}\right)+\left(D_{-1} p_{k}\right)\left[D_{\nu}(q, t)\right] .
$$

Since the definition in I.4 gives

$$
D_{\mu}\left(q^{k}, t^{k}\right)=D_{\nu}\left(q^{k}, t^{k}\right)+\left(1-t^{k}\right)\left(1-q^{k}\right)\left(T_{\mu} / T_{\nu}\right)^{k},
$$

using 1.48 and 1.46 in 1.47 we obtain

$$
\left(1-t^{k}\right)\left(1-q^{k}\right) \sum_{\mu \leftarrow \nu} d_{\mu \nu}(q, t)\left(T_{\mu} / T_{\nu}\right)^{k}=\left(D_{-1} p_{k}\right)\left[D_{\nu}(q, t)\right]
$$

Now, the first of $\mathrm{I} .10$ for $k=-1$ gives

$$
D_{-1} p_{k}=\left.\left(p_{k}+\frac{\left(1-t^{k}\right)\left(1-q^{k}\right)}{z^{k}}\right) \sum_{m \geq 0}(-z)^{m} e_{m}[X]\right|_{z^{-1}}=\left(1-t^{k}\right)\left(1-q^{k}\right)(-1)^{k-1} e_{k-1}[X] .
$$

Using this in 1.49 and carrying out the necessary cancellations completes the proof of 1.40 .

The remarkable fact that the identity in I.17 permits us to evaluate these sums without explicit knowledge of the coefficients $c_{\mu \nu}(q, t)$ and $d_{\mu \nu}(q, t)$ suggests that this idea may be extended to cases where such explicit knowledge is not available. To be precise, for a homogeneous symmetric function $f \in \Lambda^{=k}$ let us denote by $\delta_{f}^{*}$ the 
operator which is the $*$-adjoint of multiplication by $f$. From the Macdonald Pieri rules we derive that there are certain rational functions $c_{\mu \nu}(q, t)$ giving, for every $\mu \vdash n \geq k$

$$
\delta_{f}^{*} \tilde{H}_{\mu}[X ; q, t]=\sum_{\nu \subseteq_{k} \mu} c_{\mu \nu}^{f}(q, t) \tilde{H}_{\nu}[X ; q, t],
$$

where the symbol " $\nu \subseteq_{k} \mu$ " is to represent that the sum has to be carried out over partitions $\nu \subseteq \mu$ where the difference $\mu / \nu$ consists of precisely $k$ cells. Similarly, we have rational functions $d_{\mu \nu}^{f}(q, t)$ giving

$$
f[X] \tilde{H}_{\nu}[X ; q, t]=\sum_{\mu \supseteq_{k} \nu} d_{\mu \nu}^{f}(q, t) \tilde{H}_{\mu}[X ; q, t] .
$$

Without explicit knowledge of these coefficients, the method illustrated above yields identities which are entirely analogous to 1.41 and 1.42 . The result for the $c_{\mu \nu}^{f}(q, t)$ is the ricest in this case and it can be stated as follows.

Theorem 1.5. For any $P \in \Lambda$ we have

$$
\sum_{\nu \subseteq_{k} \mu} c_{\mu \nu}^{f}(q, t) P\left[D_{\nu}(q, t)\right]=\left(\nabla^{-1} f[X-\epsilon] \nabla P\right)\left[D_{\mu}(q, t)\right]
$$

Proof. Proceeding as before we use 1.43 and write

$$
\begin{aligned}
\sum_{\nu \subseteq_{k} \mu} c_{\mu \nu}^{f}(q, t) P\left[D_{\nu}(q, t)\right] & =\sum_{\nu \subseteq_{k} \mu} c_{\mu \nu}^{f}(q, t) \Pi_{F}\left[D_{\nu}(q, t)\right] \\
& =\sum_{\nu \subseteq_{k} \mu} c_{\mu \nu}^{f}(q, t)\left\langle F, \mathcal{T}_{1} \tilde{H}_{\nu}\right\rangle_{*} \\
& =\left\langle F, \mathcal{T}_{1} \delta_{f}^{*} \tilde{H}_{\mu}\right\rangle_{*}=\left\langle F, \delta_{f}^{*} \mathcal{T}_{1} \tilde{H}_{\mu}\right\rangle_{*} \\
& =\left\langle f F, \mathcal{T}_{1} \tilde{H}_{\mu}\right\rangle_{*}=\Pi_{f F}\left[D_{\mu}(q, t)\right]
\end{aligned}
$$

Now we have

$$
\Pi_{f F}=\nabla^{-1} \mathcal{T}_{\epsilon}^{-1} f \mathcal{T}_{\epsilon} \nabla P=\nabla^{-1} f[X-\epsilon] \nabla P .
$$

Using; this in 1.53 yields 1.52 and completes our argument.

2. Nabla at $\mathbf{t}=1$ and $\mathbf{q}$-Lagrange inversion. A natural integral basis for the space $\Lambda_{Z[q]}$ is given by the symmetric functions

$$
\left\{\tilde{H}_{\mu}[X ; q, 1]\right\}_{\mu} .
$$

This given, we simply define $\left.\nabla\right|_{t=1}$ to be the operator $\tilde{\nabla}$ obtained by setting

$$
\tilde{\nabla} \tilde{H}_{\mu}[X ; q, 1]=\left(\nabla \tilde{H}_{\mu}[X ; q, t]\right)_{t=1}=q^{n\left(\mu^{\prime}\right)} \tilde{H}_{\mu}[X ; q, 1] .
$$

Now we have the following basic fact.

THEOREM 2.1. The operator $\tilde{\nabla}$ is multiplicative on $\Lambda_{Z[q]}$ and may be computed by setting for all $m \geq 0$

$$
\tilde{\nabla} h_{m}\left[\frac{X}{1-q}\right]=q^{\left(\begin{array}{c}
m \\
2
\end{array}\right)} h_{m}\left[\frac{X}{1-q}\right] .
$$


Proof. It follows from [22] (p. 364 ex. 7) that the symmetric functions in 2.1 are given by the formula

$$
\tilde{H}_{\mu}[X ; q, 1]=\prod_{i=1}^{l(\mu)}(q ; q)_{\mu_{i}} h_{\mu_{i}}\left[\frac{X}{1-q}\right]
$$

Since we may write

$$
q^{n\left(\mu^{\prime}\right)}=\prod_{i=1}^{l(\mu)} q^{\left(\begin{array}{c}
\mu_{i} \\
2
\end{array}\right)}
$$

we see that the combination of $2.2,2,4$ and 2.5 yields

$$
\tilde{\nabla} \prod_{i=1}^{l(\mu)}(q ; q)_{\mu_{i}} h_{\mu_{i}}\left[\frac{X}{1-q}\right]=\prod_{i=1}^{l(\mu)}(q ; q)_{\mu_{i}} q^{\left(\begin{array}{c}
\mu_{i} \\
2
\end{array}\right)} h_{\mu_{i}}\left[\frac{X}{1-q}\right]
$$

or better

$$
\tilde{\nabla} \prod_{i=1}^{l(\mu)} h_{\mu_{i}}\left[\frac{X}{1-q}\right]=\prod_{i=1}^{l(\mu)} q^{\left(\begin{array}{c}
\mu_{i} \\
2
\end{array}\right)} h_{\mu_{i}}\left[\frac{X}{1-q}\right] .
$$

This proves the multiplicativity as well as 2.3 .

Theorem 1.2 has a number of truly remarkable consequences. To begin with let us recall that, the reformulation in terms of $\nabla$, of the conjectured Frobenius characteristic $D H_{n}[X ; q, t]$ of the module of $S_{n}$ diagonal harmonics reduces to the formula

$$
D H_{n}[X ; q, t]=\nabla e_{n}
$$

In particular, since we have the expansion

$$
e_{n}=\sum_{\mu \vdash n}\left(\prod_{i} h_{\mu_{i}}\left[\frac{x}{(1-q)}\right]\right) f_{\mu}[1-q]
$$

(with $f_{\mu}$ the forgotten basis element indexed by $\mu$ ), setting $t=1$ in 2.6 and using Theorem 2.1 we immediately obtain that

$$
D H_{n}[X ; q, 1]=\tilde{\nabla} e_{n}=\sum_{\mu \vdash n}\left(\prod_{i} q^{\left(\frac{\mu_{i}}{2}\right)} h_{\mu_{i}\left[\frac{x}{(1-q)}\right]}\right) f_{\mu}[1-q]
$$

This result leads to the following beautiful corollary of Theorem 2.1. 
'Theorem 2.2. For any $\lambda \vdash n$ we have

$$
\tilde{\nabla} S_{\lambda}[X]=\operatorname{det}\left\|D H_{\lambda_{i}^{\prime}+j-i}[X ; q, 1]\right\|_{i, j=1}^{l\left(\lambda^{\prime}\right)} \text {. }
$$

Moreover, equating coefficients of $S_{1^{n}}$ in the Schur function expansion of both sides gives

$$
\left.\tilde{\nabla} S_{\lambda}[X]\right|_{e_{n}}=\operatorname{det}\left\|C_{\lambda_{i}^{\prime}+j-i}(q)\right\|_{i, j=1}^{l\left(\lambda^{\prime}\right)} .
$$

where $\left\{C_{m}(q)\right\}_{m}$ is the q-analogue of the Catalan sequence defined by the recurrence

$$
C_{m}(q)=\sum_{k=1}^{m} q^{k-1} C_{k-1}(q) C_{m-k}(q) \quad\left(\text { with } C_{o}=1\right)
$$

Proof. Applying $\tilde{\nabla}$ to both sides of the Jacobi-Trudi identity

$$
S_{\lambda}[X]=\operatorname{det}\left\|e_{\lambda_{i}^{\prime}+j-i}[X]\right\|_{i, j=1}^{l\left(\lambda^{\prime}\right)}
$$

immediately gives 2.8 because of 2.7 and the multiplicativity of $\tilde{\nabla}$. Now 2.8 and the Pieri rules yield

$$
\left.\tilde{\nabla} S_{\lambda}[X]\right|_{S_{1^{n}}}=\operatorname{det}\left\|\left.D H_{\lambda_{i}^{\prime}+j-i}[X ; q, 1]\right|_{S_{1^{\lambda_{i}^{\prime}+j-i}}}\right\|_{i, j=1}^{l\left(\lambda^{\prime}\right)}
$$

and 2.9 follows from the fact (proved in [11]) that for each $m$

$$
\left.D H_{m}[X ; q, 1]\right|_{S_{1 m}}=C_{m}(q) \text {. }
$$

FiEMARK 2.1. We should point out that it was precisely the relation in 2.9 that permitted M. Bousquet-Melou to identify the sign occurring in formula I.8 of Conjecture I. More recently, C. Lenart (see [20]), using formula 2.8 was able to obtain a combinatorial proof of the Schur positivity of the symmetric function $(-1)^{\iota\left(\lambda^{\prime}\right)} \tilde{\nabla} S_{\lambda}$ thereby establishing the case $t=1$ and $m=1$ of Conjecture I.

Another curious consequence of Theorem 2.1 is the relation that $\tilde{\nabla}$ has to Lagrange inversion. But before we can proceed we need to recall some notation.

The $q$-Lagrange inversion problem studied in [1], [7] and [17] may be stated as followis. We are given a formal power series

$$
F(z)=F_{1} z+F_{2} z^{2}+F_{3} z^{3}+\cdots \quad\left(\text { with } F_{1} \neq 0\right)
$$

and we are to construct the formal power series

$$
f(z)=f_{1} z+f_{2} z^{2}+f_{3} z^{3}+\cdots \quad\left(\text { with } f_{1} \neq 0\right)
$$

which solves the equation

$$
z=F_{1} f(z)+F_{2} f(z) f(z q)+F_{3} f(z) f(z q) f\left(z q^{2}\right)+\cdots .
$$

We should note at the onset that equating coefficients of $z^{n}$ in this equation, the coefficients $f_{n}$ may be uniquely recursively constructed. Thus existence and uniqueness of the solution is not in question. The problem is to find a useful explicit closed form for the solution. The solution was given a closed form in [7] in terms of two operations 
roofing and starring which may be defined as follows. Given a formal power series $\Phi(z)=\sum_{k \geq 0} \Phi_{n} z^{n}$, the "roofing" and "unroofing" of $\Phi$ are respectively defined by setting

$$
\wedge \Phi(z)=\sum_{k \geq 0} \Phi_{n} q^{-\left(\begin{array}{l}
n \\
2
\end{array}\right)} z^{n} \quad \text { and } \quad \vee \Phi(z)=\sum_{k \geq 0} \Phi_{n} q^{\left(\begin{array}{c}
n \\
2
\end{array}\right)} z^{n} .
$$

In the same vein, when $\Phi_{0}=1$, "left" and "right stars" of $\Phi(z)$ are obtained by setting

$$
{ }^{*} \Phi(z)=\Phi(z) \Phi(z / q) \Phi\left(z / q^{2}\right) \cdots \quad \text { and } \quad \Phi^{*}(z)=\Phi(z) \Phi(z q) \Phi\left(z q^{2}\right) \cdots
$$

This given, it was shown in [7] that when $F(z)$ is of the form

$$
F(z)=\frac{z}{R(z)}=\frac{z}{1+R_{1} z+R_{2} z^{2}+R_{3} z^{3}+\cdots}
$$

then the solution $f(z)$ of 2.12 is given the expression

$$
f(z)=z \frac{\mathrm{V}^{*} R(z q)}{\mathrm{V}^{*} R(z)} .
$$

This rather mysterious formula was difficult to use at the time in that there was no method to derive from 2.16 an actual expression for the coefficients $f_{n}$. These coefficients were given explicit expressions in [11] by means of the theory of symmetric functions. The reader may also find there a lattice path interpretation of 2.12 as well as its solution $f(z)$. Our purpose here is to show that $\tilde{\nabla}$ is intimately related to this $q$-Lagrange inversion problem. In fact, a number of the manipulations carried out in [7] and [11] will be seen to acquire an entirely new light by means of $\tilde{\nabla}$ and plethystic notation.

To begin with we should note that the reason we can use the theory of symmetric functions in this context is due to the fact that, given an infinite alphabet $X=$ $x_{1}+x_{2}+x_{3}+\cdots$, the elementary symmetric functions $e_{1}[X], e_{2}[X], e_{3}[X], \ldots$ are algebraically independent. Thus any formulas we may derive in the special case that $R_{n}=e_{n}[X]$ in 2.4, will remain valid when, upon expressing everything in terms of the elementary basis, we make the substitutions $e_{n}[X] \rightarrow R_{n}$. This idea will perhaps be better understood after we illustrate it by some examples.

An important ingredient in this development is that the roofing operator untangles certain "tangled" products of $q$-series. More precisely, we have the following basic identity

Proposition 2.1. For any two $q$-series $A(z)=\sum_{m \geq 0} A_{m}(q) z^{m}$ and $B(z)=$ $\sum_{m \geq 0} B_{m}(q) z^{m}$ we set

$$
(A * B)(z)=\sum_{n, m \geq 0} A_{n}(q) B_{m}(q) q^{n m} z^{n+m}=\sum_{n \geq 0} A_{n}(q) z^{n} B\left(q^{n} z\right) .
$$

This given we have

$$
\wedge(A * B)=(\wedge A) \times(\wedge B)
$$

This identity follows routinely from 2.17 and the definition in 2.13. A proof may also be found in [7]. 
Keeping this in mind, we can prove the following surprising result.

THEOREM 2.2. When

$$
F(z)=\frac{z}{\sum_{m \geq 0} e_{n}[X] z^{n}}=\frac{z}{\tilde{\Omega}[z X]}
$$

the solution of 2.12 is simply given by the formal series

$$
f(z)=z \tilde{\nabla} \tilde{\Omega}[z q X] .
$$

In particular the coefficients of $f(z)$ may be computed from the formula

$$
f_{n}=q^{n-1} \tilde{\nabla} e_{n-1}=q^{n-1} \sum_{\mu \vdash n-1}\left(\prod_{i} q^{\left(\begin{array}{c}
\mu_{i} \\
2
\end{array}\right)} h_{\mu_{i}\left[\frac{x}{(1-q)}\right]}\right) f_{\mu}[1-q] .
$$

Proof. Substituting 2.20 in the right hand side of 2.12 and using the multiplicativity of $\tilde{\nabla}$ we obtain

$$
\begin{aligned}
\sum_{n: 1} F_{n} f(z) \cdots f\left(z q^{n-1} z\right) & =\sum_{m \geq 1} F_{n} z^{n} q^{\left(\begin{array}{c}
n \\
2
\end{array}\right)} \tilde{\nabla} \tilde{\Omega}[z q X] \tilde{\Omega}\left[z q^{2} X\right] \cdots \tilde{\Omega}\left[z q^{n} X\right] \\
& =\sum_{n \geq 1} F_{n} z^{n} q^{\left(\begin{array}{c}
n \\
2
\end{array}\right)} \tilde{\nabla} \tilde{\Omega}\left[z q \frac{1-q^{n}}{1-q} X\right] \\
& =\sum_{n \geq 1} F_{n} z^{n} q^{\left(\begin{array}{c}
n \\
2
\end{array}\right)} \tilde{\nabla} \tilde{\Omega}\left[z q \frac{1}{1-q} X\right] / \tilde{\Omega}\left[z q \frac{q^{n}}{1-q} X\right] \\
& =\left(\tilde{\nabla} \tilde{\Omega}\left[z q \frac{1}{1-q} X\right]\right) \sum_{n \geq 1} F_{n} z^{n} q^{\left(\begin{array}{c}
n \\
2
\end{array}\right)} / \tilde{\nabla} \tilde{\Omega}\left[z q \frac{q^{n}}{1-q} X\right] .
\end{aligned}
$$

Thus to show 2.12 we need only verify that

$$
\frac{z}{\tilde{\nabla} \tilde{\Omega}\left[z q \frac{1}{1-q} X\right]}=\sum_{n \geq 1} \frac{F_{n} z^{n} q^{\left(\begin{array}{c}
n \\
2
\end{array}\right)}}{\tilde{\nabla} \tilde{\Omega}\left[z q \frac{q^{n}}{1-q} X\right]} .
$$

Now the multiplicativity of $\tilde{\nabla}$ allows us to rewrite this relation in the form

$$
z \tilde{\nabla} \tilde{\Omega}\left[-z q \frac{1}{1-q} X\right]=\sum_{n \geq 1} F_{n} z^{n} q^{\left(\begin{array}{c}
n \\
2
\end{array}\right)} \tilde{\nabla} \tilde{\Omega}\left[-z q \frac{q^{n}}{1-q} X\right] .
$$

Or better yet, since for any alphabet $Y$ we have $\tilde{\Omega}[-Y]=\Omega[\epsilon Y]$ we are reduced to showing that

$$
z \tilde{\nabla} \Omega\left[\epsilon z q \frac{1}{1-q} X\right]=\sum_{n \geq 1} F_{n} z^{n} q^{\left(\begin{array}{c}
n \\
2
\end{array}\right)} \tilde{\nabla} \Omega\left[\epsilon z q \frac{q^{n}}{1-q} X\right] .
$$

We can now "roof" both sides and obtain

$$
z \tilde{\nabla} \wedge\left(\Omega\left[\epsilon z \frac{1}{1-q} X\right]\right)=\left(\sum_{n \geq 1} F_{n} z^{n}\right) \tilde{\nabla} \wedge \Omega\left[\epsilon z q \frac{1}{1-q} X\right] .
$$

Recalling the definition of $\tilde{\nabla}$, this is simply

$$
z \sum_{m \geq 0}(\epsilon z)^{m} h_{m}\left[\frac{X}{1-q}\right]=\left(\sum_{n \geq 1} F_{n} z^{n}\right)\left(\sum_{m \geq 0}(\epsilon z)^{m} h_{m}\left[\frac{q X}{1-q}\right]\right)
$$


which may also be written as

$$
\frac{F(\epsilon z)}{\epsilon z}=\frac{\Omega\left[z X \frac{q}{1-q}\right]}{\Omega\left[z X \frac{1}{1-q}\right]}=\Omega[-z X]
$$

and this is in complete agreement with the choice we made in 2.19.

Another curious result established in [7], whose nature is better understood by means of $\tilde{\nabla}$, can be stated as follows.

THEOREM 2.3. If $F(z)$ and $f(z)$ are two formal power series related by 2.12, then for two sequences of constants $\left\{\Phi_{n}\right\}_{n \geq 0},\left\{\Psi_{n}\right\}_{n \geq 0}$ we have

$$
\sum_{n \geq 0} \Phi_{n} f(z) f(z q) \cdots f\left(z q^{n-1}\right)=\sum_{m \geq 0} \Psi_{m} z^{n}
$$

if and only if

$$
\sum_{n \geq 0} \Phi_{n} z^{n}=\sum_{m \geq 0} \Psi_{m} F(z) F(z / q) \cdots F\left(z / q^{n-1}\right) .
$$

$\tilde{\nabla}$-Proof. Using 2.20 we may write the left hand side of 2.23 in the forms

$$
\begin{aligned}
\sum_{n \geq 0} \Phi_{n} z^{n} q^{\left(\begin{array}{c}
n \\
2
\end{array}\right)} \tilde{\nabla} \tilde{\Omega}[z q X] \tilde{\nabla} \tilde{\Omega}\left[z q^{2} X\right] \cdots \tilde{\nabla} \tilde{\Omega}\left[z q^{n} X\right] \\
=\sum_{n \geq 0} \Phi_{n} z^{n} q^{\left(\begin{array}{c}
n \\
2
\end{array}\right)} \tilde{\nabla} \tilde{\Omega}\left[\frac{1-q^{n}}{1-q} z q X\right] \\
=\sum_{n \geq 0} \Phi_{n} z^{n} q^{\left(\begin{array}{c}
n \\
2
\end{array}\right)} \tilde{\nabla} \tilde{\Omega}\left[\frac{-q^{n+1}}{1-q} z X\right] / \tilde{\nabla} \tilde{\Omega}\left[\frac{-q}{1-q} z X\right]
\end{aligned}
$$

Thus 2.23 is equivalent to

$$
\sum_{n \geq 0} \Phi_{n} z^{n} q^{\left(\begin{array}{c}
n \\
2
\end{array}\right)} \tilde{\nabla} \tilde{\Omega}\left[\frac{-q^{n+1}}{1-q} z X\right]=\left(\tilde{\nabla} \tilde{\Omega}\left[\frac{-q}{1-q} z X\right]\right) \sum_{m \geq 0} \Psi_{m} z^{m}
$$

Using again the fact that for any $A$ we have $\tilde{\Omega}[-A]=\Omega[\epsilon A]$, roofing both sides of 2.25 yields

$$
\left(\sum_{n \geq 0} \Phi_{n} z^{n}\right) \wedge \tilde{\nabla} \Omega\left[\frac{q}{1-q} \epsilon z X\right]=\wedge\left(\left(\tilde{\nabla} \Omega\left[\frac{q}{1-q} \epsilon z X\right]\right) \sum_{m \geq 0} \Psi_{m} z^{m}\right)
$$


Now we have

$$
\begin{aligned}
& \wedge\left(\left(\tilde{\nabla} \Omega\left[\frac{q}{1-q} \epsilon z X\right]\right) \sum_{m \geq 0} \Psi_{m} z^{m}\right) \\
& =\sum_{n, m \geq 0} q^{\left(\begin{array}{c}
n \\
2
\end{array}\right)} h_{n}\left[\frac{q X}{1-q}\right] \epsilon^{n} \Psi_{m} z^{n+m} q^{-\left(\begin{array}{c}
n+m \\
2
\end{array}\right)} \\
& =\sum_{n, m \geq 0} h_{n}\left[\frac{-X}{1-1 / q}\right](\epsilon z)^{n} q^{-n m} \Psi_{m} q^{-\left(\begin{array}{c}
m \\
2
\end{array}\right)} z^{m} \\
& =\sum_{m \geq 0} \Psi_{m} q^{-\left(\begin{array}{c}
m \\
2
\end{array}\right)} z^{m} \Omega\left[-\epsilon z \frac{q^{-m} X}{1-1 / q}\right] \\
& =\sum_{m \geq 0} \Psi_{m} q^{-\left(\begin{array}{c}
m \\
2
\end{array}\right)} z^{m} \tilde{\Omega}\left[z \frac{q^{-m} X}{1-1 / q}\right]
\end{aligned}
$$

and

$$
\begin{aligned}
\wedge \tilde{\nabla} \Omega\left[\frac{q}{1-q} \epsilon z X\right] & =\sum_{n \geq 0} q^{\left(\begin{array}{c}
n \\
2
\end{array}\right)} h_{n}\left[\frac{q X}{1-q}\right] q^{-\left(\begin{array}{c}
n \\
2
\end{array}\right)}(\epsilon z)^{n} \\
& =\sum_{n \geq 0} h_{n}\left[\frac{-X}{1-1 / q}\right](\epsilon z)^{n} \\
& =\Omega\left[\frac{-\epsilon z X}{1-1 / q}\right]=\tilde{\Omega}\left[\frac{z X}{1-1 / q}\right]
\end{aligned}
$$

Using 2.27 and 2.28 in 2.26 gives

$$
\left(\sum_{n \geq 0} \Phi_{n} z^{n}\right) \tilde{\Omega}\left[\frac{z X}{1-1 / q}\right]=\sum_{m \geq 0} \Psi_{m} q^{-\left(\begin{array}{c}
m \\
2
\end{array}\right)} z^{m} \tilde{\Omega}\left[z \frac{q^{-m} X}{1-1 / q}\right]
$$

and this can be rearranged to

$$
\sum_{n \geq 0} \Phi_{n} z^{n}=\sum_{m \geq 0} \Psi_{m} q^{-\left(\begin{array}{c}
m \\
2
\end{array}\right)} \frac{z^{m}}{\tilde{\Omega}\left[z \frac{1-q^{-m} X}{1-1 / q}\right]}=\sum_{m \geq 0} \Psi_{m} \frac{z}{\tilde{\Omega}[z X]} \frac{z / q}{\tilde{\Omega}[z X / q]} \cdots \frac{z / q^{n-1}}{\tilde{\Omega}\left[z X / q^{n-1}\right]}
$$

proving the equivalence of 2.23 and 2.24 .

It will be good to illustrate how these symmetric function identities can be used in $q$-enumeration. To this end let us recall that a "Lukaciewicz" path of length $n$ is a lattice path which starts at the origin and ends at $(n, 0)$, always remaining weakly above the the $x$-axis. Its steps are also restricted to be the vectors

$$
v_{k}=(1, k-1) \quad(\text { for } k \geq 0) .
$$

Calling $\mathcal{L}$ the collection of all such paths, we associate to each path $\pi \in \mathcal{L}$ the "weight"

$$
w(\pi)=q^{A(\pi)} z^{n(\pi)} x_{1}^{m_{1}(\pi)} x_{2}^{m_{2}(\pi)} x_{3}^{m_{3}(\pi)} \ldots
$$

where $A(\pi)$ denotes the area between the path and the $x$-axis, $n(\pi)$ is the number of steps of $\pi$ and $m_{k}(\pi)$ denotes the number of times $\pi$ takes the step $v_{k}$. Now it is well known and easy to show that the formal power series

$$
L(z)=\sum_{\pi \in \mathcal{L}} w(\pi)
$$


satisfies the following equation:

$L(z)=1+z x_{1} L(z)+z^{2} q^{\left(\begin{array}{l}2 \\ 2\end{array}\right)} x_{2} L(z) L(z q)+\cdots+z^{k} q^{\left(\begin{array}{c}k \\ 2\end{array}\right)} x_{k} L(z) L(z q) \cdots L\left(z q^{k-1}\right)+\cdots$.

Setting $g(z)=z L(z)$, we are led to the equation

$$
g(z)=z\left(1+x_{1} g(z)+x_{2} g(z) g(z q)+\cdots+x_{k} g(z) g(z q) \cdots g\left(z q^{k-1}\right)+\cdots\right) .
$$

Note next that if we apply Theorem 2.3 to the equation 2.12 , we derive that we must also have

$$
\sum_{n \geq 1} f_{n} F(z) F(z / q) \cdots F\left(z / q^{n-1}\right)=z
$$

Now in the case that $F(z)$ is given by 2.15 we can rewrite this as

$$
\sum_{n \geq 1} f_{n} F(z / q) \cdots F\left(z / q^{n-1}\right)=R(z)=1+R_{1} z+R_{2} z^{2}+\cdots+R_{k} z^{k}+\cdots .
$$

The replacement $z \rightarrow q z$ then gives

$$
\sum_{n \geq 1} f_{n} F(z) \cdots F\left(z / q^{n-2}\right)=R(z)=1+R_{1} q z+R_{2} q^{2} z^{2}+\cdots+R_{k} q^{k} z^{k}+\cdots
$$

and a further use of Theorem 2.3 yields the equivalent equation

$$
\sum_{n \geq 1} f_{n} z^{n-1}=\sum_{k \geq 0} R_{k} q^{k} f(z) f(z q) \cdots f\left(z q^{k-1}\right) .
$$

This is

$f(z)=z\left(1+R_{1} q f(z)+R_{2} q^{2} f(z) f(z q)+\cdots+R_{k} q^{k} f(z) f(z q) \cdots f\left(z q^{k-1}\right)+\cdots\right)$.

Comparing with 2.29 , we see that the $q$-enumerator $L(z)$ of Lukaciewicz paths by area may be obtained by solving 2.12 when $F(z)$ is given by 2.15 with

$$
R_{k}=x_{k} / q^{k} \quad(\text { for } k \geq 1) \text {. }
$$

To be precise we have

$$
L(z)=f(z) / z
$$

Thus if we denote by $\mathcal{L}_{n}$ the collection of Lukaciewicz paths with $n$ steps, formula 2.21 gives that

$$
\sum_{\pi \in \mathcal{L}_{n}} q^{A(\pi)} x_{1}^{m_{1}(\pi)} x_{2}^{m_{2}(\pi)} x_{3}^{m_{3}(\pi)} \cdots=\left.q^{n} \sum_{\mu \vdash n}\left(\prod_{i} q^{\left(\mu_{i}\right)} h_{\mu_{i}\left[\frac{x}{(1-q)}\right]}\right) f_{\mu}[1-q]\right|_{e_{i} \rightarrow x_{i} / q^{i}}
$$

where it is to be understood that the replacements indicated by the symbol " $\left.\right|_{e_{i} \rightarrow x_{i} / q^{i}}$ " should be carried out after the preceding symmetric function has been expanded in terms of the elementary basis. We should also mention that it can be shown (see [11]) that for $\mu=1^{\alpha_{1}} 2^{\alpha_{2}} 3^{\alpha_{3}} \ldots$ a $k$-part partition of $n$ we have

$$
f_{\mu}[1-q]=(-1)^{n-k} \frac{(k-1) !}{\prod_{i} \alpha_{i} !} \sum_{i=1}^{k}\left(1-q^{\mu_{i}}\right) .
$$


The special cases where the paths are restricted to have only certain selected subsets of steps can be obtained from 2.30 by setting to 0 the appropriate subset of $x_{k}$. For instance, the polynomial below

$$
\begin{gathered}
x_{1}{ }^{6}+\left(q^{4}+2 q^{3}+3 q^{2}+4 q+5\right) q x_{2} x_{1}{ }^{4} \\
+\left(q^{6}+q^{5}+3 q^{4}+3 q^{3}+5 q^{2}+3 q+4\right) q^{3} x_{3} x_{1}{ }^{3} \\
+\left(q^{6}+2 q^{5}+5 q^{4}+4 q^{3}+6 q^{2}+6 q+6\right) q^{2} x_{2}{ }^{2} x_{1}{ }^{2} \\
+\left(q^{6}+q^{5}+2 q^{4}+3 q^{3}+3 q^{2}+2 q+3\right) q^{6} x_{4} x_{1}{ }^{2} \\
+\left(q^{7}+3 q^{6}+2 q^{5}+6 q^{4}+4 q^{3}+4 q^{2}+4 q+6\right) q^{4} x_{2} x_{3} x_{1} \\
+\left(q^{6}+q^{4}+2 q^{2}+1\right) q^{3} x_{2}{ }^{3} \\
+\left(q^{4}+1\right)\left(q^{2}+2\right) q^{7} x_{2} x_{4} \\
+\left(q^{6}+q^{3}+1\right) q^{6} x_{3}{ }^{2}
\end{gathered}
$$

was obtained from 2.30 by setting $n=6, e_{1}=x_{1} / q, e_{2}=x_{2} / q^{2}, e_{3}=x_{3} / q^{3}$, $e_{4}=x_{3} / q^{4}$ and $e_{5}=e_{6}=0$. Accordingly it $q$-enumerates by area the collection of Lukaciewicz paths of length 6 which only use steps $v_{0}, v_{1}, v_{2}, v_{3}$ and $v_{4}$. Of course such polynomials may also be readily computed via the recursions that they necessarily satisfy. What is interesting is that the theory of symmetric functions provides the tools for constructing them quite explicitly.

REMARK 2.2. It stands to reason that $\nabla$ itself must yield some $q, t$ version of Lagrange inversion. In fact, starting again with $F(z)=z / \tilde{\Omega}[z X]$, we might call the $q, t$-inverse of $F(z)$ a power series $f(z)$ of the form

$$
f(z)=z \sum_{n \geq 0} c_{n}(q, t) \nabla e_{n} z^{n}
$$

for a suitable choice of the coefficients $c_{n}(q, t)$. Of course, since $\nabla$ is symmetric in $q, t$, whatever we showed for the specialization at $t=1$ goes through verbatim (by interchanging $q$ and $t$ ) for the specialization at $q=1$. So in the former case we take $c_{n}(q, t)=q^{n}$ and in the latter $c_{n}(q, t)=t^{n}$. Curiously enough, we find that the specialization at $t=1 / q$ of 2.32 can also be related to Lagrange inversion if we take $c_{n}(q, \dot{t})=q^{\left(\begin{array}{l}n \\ 2\end{array}\right)}$. In fact, it was shown in [11] that

$$
\left.q^{\left(\begin{array}{c}
n \\
2
\end{array}\right)} \nabla e_{n}\right|_{t=1 / q}=\frac{e_{n}\left[X\left(1+q+\cdots+q^{n}\right)\right]}{1+q+\cdots+q^{n}} .
$$

Thus the formal series in 2.32 with this specialization becomes

$$
f(z)=z \sum_{n \geq 0} \frac{e_{n}\left[X\left(1+q+\cdots+q^{n}\right)\right]}{1+q+\cdots+q^{n}} z^{n} .
$$

To recognize the nature of the connection with Lagrange inversion in this case, we need only point out that we may write

$$
e_{n}\left[X\left(1+q+\cdots+q^{n}\right)\right]=\left.\tilde{\Omega}[z X] \tilde{\Omega}[z q X] \cdots \tilde{\Omega}\left[z q^{n} X\right]\right|_{z^{n}}
$$


Thus 2.34 may be viewed as

$$
f_{n+1}=\left.\sum_{n \geq 0} \frac{1}{1+q+\cdots+q^{n}} \tilde{\Omega}[z X] \tilde{\Omega}[z q X] \cdots \tilde{\Omega}\left[z q^{n} X\right]\right|_{z^{n}}
$$

or better yet (equating 2.15 and 2.19 ) this is simply

$$
f_{n+1}=\left.\sum_{n \geq 0} \frac{1}{1+q+\cdots+q^{n}} R(z) R(z q) \cdots R\left(z q^{n}\right)\right|_{z^{n}},
$$

which is easily seen to reduce to the classical Lagrange inversion formula for $q=1$. In summary $\nabla$ offers a way of interpolating, by means of the interplay between $q$ and $t$, between two quite different yet natural $q$-analogues of Lagrange inversion, namely, between the one which affords a combinatorial interpretation of the solution and one which simply exhibits a $q$-analogue of the solution formula. Only time will tell if there is a "natural" explanation for this curious fact.

3. Positivity properties. In this section we shall present a variety of conjectures which resulted from experimenting with $\nabla$ and related operators. To simplify the statement of our results we need to introduce some terminology. Here and in the following when we say that a certain symmetric function $F$ is "Schur integral" we simply mean that it has a Schur function expansion with coefficients in $\mathbf{Z}[q, t]$. If the coefficients are in $\mathbf{N}[q, t]$ then we shall say that $F$ is "Schur positive integral" or briefly "Schur positive". If $F$ or $-F$ is Schur positive, we shall say that $F$ is "virtually positive". If the elementary basis expansion of $F$ has coefficients in $\mathbf{N}[q, t]$ we shall say that $F$ is "e-positive". Of course e-positivity is stronger than Schur positivity. The results of last sections essentially state that $q^{n} \tilde{\nabla} e_{n}$ is e-positive. In this particular case the result could be established by giving a combinatorial interpretation to the coefficients. However, this is one of the very few positivity findings that can be actually proved. In most cases the best we can do is offer a "proof" based on some stronger and more general conjecture. For instance, there is relatively simple algorithm which constructs the polynomials $\tilde{H}_{\mu}[X ; q, t]$, based on the fact that for any $\mu$ we have the triangularity relations:

$$
\begin{gathered}
\tilde{H}_{\mu}[X ; q, t]=\tilde{h}_{\mu}(q, t) S_{\mu}\left[\frac{X}{1-t}\right]+\sum_{\lambda<\mu} S_{\lambda}\left[\frac{X}{1-t}\right] a_{\lambda \mu}(q, t) \\
\tilde{H}_{\mu}[X ; q, t]=\tilde{h}_{\mu}(q, t) S_{\mu}\left[\frac{X}{1-q}\right]+\sum_{\lambda>\mu} S_{\lambda}\left[\frac{X}{1-q}\right] b_{\lambda \mu}(q, t) .
\end{gathered}
$$

If we program this on the computer we would quickly be led to the "discovery", without explicit knowledge of any the coefficients $a_{\lambda \mu}(q, t)$ or $b_{\lambda \mu}(q, t)$, that the $\tilde{H}_{\mu}{ }^{\prime} s$ are in fact Schur positive. This is essentially what is now referred to as the "Macdonald $q, t$-Kostka conjecture". However, after a decade of efforts in proving this conjecture, considerably more refined information concerning the polynomials $\tilde{H}_{\mu}$ has emerged. To begin with, Garsia-Haiman have conjectured in [8] that these polynomials are the Frobenius characteristics of certain bigraded $S_{n}$ modules. More precisely, for $\mu \vdash n$ set

$$
\Delta_{\mu}(x ; y)=\operatorname{det}\left\|x_{i}^{l_{\mu}(s)} y_{i}^{a_{\mu}(s)}\right\|_{\substack{i \in[1, n] \\ s \in \mu}}
$$


and let $\mathbf{M}_{\mu}$ be linear span of the derivatives of $\Delta_{\mu}(x ; y)$; in symbols

$$
\mathbf{M}_{\mu}=\mathcal{L}_{\delta}\left[\partial_{x}^{p} \partial_{y}^{q} \Delta_{\mu}(x ; y)\right] .
$$

Moreover let $\mathcal{H}_{r, s}\left[\mathbf{M}_{\mu}\right]$ denote the subspace of $\mathbf{M}_{\mu}$ consisting of its bihomogeneous elements of degree $r$ in $x$ and degree $s$ in $y$. Since $\mathcal{H}_{r, s}\left[\mathbf{M}_{\mu}\right]$ is invariant under the diagonal action of $S_{n}$, we can set

$$
C_{\mu}[X ; q, t]=\sum_{r=0}^{n(\mu)} \sum_{s=0}^{n\left(\mu^{\prime}\right)} t^{r} q^{s} F \operatorname{ch} \mathcal{H}_{r, s}\left[\mathbf{M}_{\mu}\right]
$$

where the symbol " $F c h$ " denotes the operation of taking the Frobenius image of a character, that is, the map that sends the character $\chi^{\lambda}$ onto the Schur function $S_{\lambda}$.

Now it was conjectured in [8] that for all $\mu$ we have

$$
C_{\mu}[X ; q, t]=\tilde{H}_{\mu}[X ; q, t] .
$$

Of course the Macdonald $q, t$-Kostka conjecture would then follow, since under this equality the coefficients $\tilde{K}_{\lambda \mu}(q, t)$ in I.21 would have to encode the manner in which the irreducible $S_{n}$-character $\chi^{\lambda}$ is distributed among the components $\mathcal{H}_{r, s}\left[\mathbf{M}_{\mu}\right]$. We shall refer to 3.5 as the " $C=\tilde{H}$ " conjecture. We should mention that 3.5 has been verified for all $\mu \vdash n \leq 8$ by computer, for $\mu$ a hook in [12], for two row partitions and for any partition obtained by adding an inner corner to a hook [23]. It can be shown by an elementary combinatorial argument that for any $\mu \vdash n$ we have (see [12])

$$
\operatorname{dim} \mathbf{M}_{\mu} \leq n !
$$

Since Macdonald in [21] showed that

$$
\left.\tilde{K}_{\lambda \mu}(q, t)\right|_{t, q=1}=f_{\lambda} \quad \text { (the number of standard tableaux of shape } \lambda \text { ) }
$$

it would immediately follows from 3.5 that we must have equality in 3.6. Surprisingly, even this tantalizingly simple assertion, which has come to be referred to as the " $n$ !conjecture", has also escaped proof after more than ten years since its formulation in [21]. What is even more surprising is the fact that Haiman in [19] proved that the validity of the $n$ ! conjecture for any given $\mu$ forces 3.5 to be true for that same $\mu$. In [3] and [2] the reader may find a variety of conjectures that strengthen and extend the $n$ ! conjecture. We shall recall here those that are most intimately related to our operator $\nabla$.

Let $\mu$ be a fixed partition of $n+1$ and let

$$
\operatorname{Pred}(\mu)=\left\{\nu^{(1)}, \nu^{(2)}, \ldots, \nu^{(d)}\right\}
$$

be the collection of partitions obtained by removing one of the corners of $\mu$. For a pair $\nu \rightarrow \mu$, it will be convenient to denote by $\mu / \nu$ the corner cell we must remove from $\mu$ to get $\nu$. To be specific, we shall assume that the partitions in 3.8 are ordered so that the corner $\mu / \nu^{(k)}$ is northwest of the corner $\mu / \nu^{(k+1)}$. For two subsets $T \subseteq S \subseteq \operatorname{Pred}(\mu)$ set

$$
\mathbf{M}_{S}^{T}=\left(\bigcap_{\alpha \in T} \mathbf{M}_{\alpha}\right) \cap\left(\left(\sum_{\beta \in S-T} \mathbf{M}_{\beta}\right) \cap\left(\bigcap_{\alpha \in T} \mathbf{M}_{\alpha}\right)\right)^{\perp}
$$


where the symbols " $\bigcap$ " and " $\sum$ " denote intersection and sum (not usually direct) of vector spaces, and " $\perp$ " denotes the operation of taking orthogonal complements with respect to the natural scalar product of polynomials in $x_{1}, \ldots, x_{n} ; y_{1}, \ldots, y_{n}$ that makes monomials into an orthogonal set. Since this scalar product is invariant under the diagonal action of $S_{n}$, we see that $\mathbf{M}_{S}^{T}$ is a well-defined $S_{n}$-module. We shall denote its bivariate Frobenius characteristic by $\phi_{S}^{T}$. One of the assertions of the SF-heuristics in [3] is that in the linear span

$$
\mathcal{L}\left[\tilde{H}_{\alpha}: \alpha \in S\right]
$$

we have $m=|S|$ Schur positive symmetric polynomials

$$
\phi_{S}^{(1)}, \phi_{S}^{(2)}, \ldots, \phi_{S}^{(m)}
$$

such that for any $T \subseteq S$ of cardinality $k$ we have

$$
\phi_{S}^{T}=\frac{\phi_{S}^{(k)}}{\prod_{\alpha \in S-T} T_{\alpha}} .
$$

It is also a consequence of the SF-heuristics that for $k=1, \ldots, m-1$ we can set

$$
\phi_{S}^{(k)}=(-\nabla)^{m-k} \phi_{S}^{(m)},
$$

while $\phi_{S}^{(m)}$ itself can be computed from the formula

$$
\phi_{S}^{(m)}=\sum_{\alpha \in S}\left(\prod_{\beta \in S /\{\alpha\}} \frac{1}{1-T_{\alpha} / T_{\beta}}\right) \tilde{H}_{\alpha}=\sum_{\alpha \in S}\left(\prod_{\beta \in S /\{\alpha\}} \frac{1}{1-\nabla / T_{\beta}}\right) \tilde{H}_{\alpha}
$$

To be consistent with the notation we adopted in [3] we shall use the symbols $\phi_{\mu}$ or $\phi_{\mu}^{(k)}$ to denote $\phi_{S}^{(m)}$ or $\phi_{S}^{(k)}$ when $S=\mathcal{P r e d}(\mu)$. In this vein, it will also be convenient to set, for any subset $S \subseteq \mathcal{P}$ red $(\mu)$,

$$
{ }^{c} S=\operatorname{Pred}(\mu)-S .
$$

Now it is shown in [3] that

$$
\phi_{S}^{(m)}=\left(\prod_{\beta \in{ }^{c} S}\left(1-\frac{\nabla}{T_{\beta}}\right)\right) \phi_{\mu} .
$$

In particular, when $S$ consists of a single partition $\nu^{(i)} \in \mathcal{P} r e d(\mu)$, this reduces to

$$
\tilde{H}_{\nu^{(i)}}(x ; q, t)=\left(\prod_{j=1 ; j \neq i}^{d}\left(1-\frac{\nabla}{T_{\nu^{(j)}}}\right)\right) \phi_{\mu}
$$

which may also be rewritten in the form (see 3.19 of [3])

$$
\tilde{H}_{\nu^{(i)}}(x ; q, t)=\sum_{k=1}^{d} \phi_{\mu}^{(k)} e_{d-k}\left[\frac{1}{T_{\nu^{(1)}}}+\frac{1}{T_{\nu^{(2)}}}+\cdots+\frac{1}{T_{\nu^{(d)}}}-\frac{1}{T_{\nu^{(i)}}}\right] .
$$

Finally note that if $\nu^{(i)}=\alpha \in S$ then by combining 3.12 and 3.13 we can also write

$$
\tilde{H}_{\alpha}(x ; q, t)=\prod_{\beta \in S ; \beta \neq \alpha}\left(1-\frac{\nabla}{T_{\beta}}\right) \prod_{\beta \in{ }^{c} S}\left(1-\frac{\nabla}{T_{\beta}}\right) \phi_{\mu}=\prod_{\beta \in S ; \beta \neq \alpha}\left(1-\frac{\nabla}{T_{\beta}}\right) \phi_{S}^{(m)}
$$


or equivalently, for $S=\left\{\alpha^{(1)}, \alpha^{(2)}, \ldots, \alpha^{(m)}\right\}$ and $\alpha=\alpha^{(i)}$

$$
\tilde{H}_{\alpha^{(i)}}(x ; q, t)=\sum_{k=1}^{m} \phi_{S}^{(k)} e_{m-k}\left[\frac{1}{T_{\alpha^{(1)}}}+\frac{1}{T_{\alpha^{(2)}}}+\cdots+\frac{1}{T_{\alpha^{(m)}}}-\frac{1}{T_{\alpha^{(i)}}}\right] .
$$

To complete the picture we need to recall that in [3] the weights of the corners

$$
\mu / \nu^{(1)}, \mu / \nu^{(2)}, \ldots, \mu / \nu^{(d)}
$$

were respectively called

$$
x_{1}, x_{2}, \ldots, x_{d}
$$

Moreover, if

$$
x_{i}=t^{l_{i}^{\prime}} q^{a_{i}^{\prime}}
$$

then we also set

$$
u_{i}=t^{l_{i+1}^{\prime}} q^{a_{i}^{\prime}} \quad(\text { for } i=1,2, \ldots, d-1)
$$

We shall refer to the $u_{i}$ as the weights of the "inner corners" of $\mu$. Finally we set

$$
u_{0}=t^{l_{1}^{\prime}} / q, u_{d}=q^{a_{d}^{\prime}} / t \text { and } x_{0}=1 / t q .
$$

It was shown in [15] that the products in 1.38 giving the coefficients $c_{\mu \nu}(q, t)$ undergo massive cancellations which reduce them to relatively simpler expressions in terms of the corner weights. This results in the formula

$$
c_{\mu \nu^{(i)}}=\frac{1}{\tilde{M}} \frac{1}{x_{i}} \frac{\prod_{j=0}^{d}\left(x_{i}-u_{j}\right)}{\prod_{j=1 ; j \neq i}^{d}\left(x_{i}-x_{j}\right)}
$$

Taking account of the fact that $x_{i} T_{\nu^{(i)}}=T_{\mu}$, formula 3.13 can then also be written in the form

$$
\tilde{H}_{\nu^{(i)}}(x ; q, t)=\prod_{j=1 ; j \neq i}^{d}\left(1-\nabla \frac{x_{i}}{T_{\mu}}\right) \phi_{\mu} .
$$

This given, from 3.19 and 3.20 we can derive the following beautiful identities: $\left.{ }^{(}\right)$ (3.21)

$$
\begin{aligned}
& \text { a) } \partial_{p_{1}} \tilde{H}_{\mu}=\frac{1}{M} \frac{T_{\mu}}{\nabla}\left(\prod_{s=0}^{d}\left(1-\nabla \frac{u_{s}}{T_{\mu}}\right)\right) \phi_{\mu} \\
& \text { b) } \partial_{p_{1}} \tilde{H}_{\mu}=\sum_{k=1}^{d} \frac{\phi_{S}^{(k)}}{T_{\mu}^{m-k}} \frac{e_{m+1-k}\left[x_{0}+\cdots+x_{d}\right]-e_{m+1-k}\left[u_{0}+\cdots+u_{d}\right]}{M} .
\end{aligned}
$$

We should note that one of the consequences of 3.11 is that for any two predecessors $\alpha$ and $\beta$ of a partition $\mu \vdash n+1$ the symmetric function

$$
\Phi_{\alpha \beta}[X ; q, t]=\frac{T_{\beta} \tilde{H}_{\alpha}[X ; q, t]-T_{\alpha} \tilde{H}_{\beta}[X ; q, t]}{T_{\beta}-T_{\alpha}}
$$


is the conjectured Frobenius characteristic of the intersection of the two $S_{n}$-modules $\mathbf{M}_{\alpha}$ and $\mathbf{M}_{\beta}$ and therefore it should always be Schur positive. It is another consequence of the Science Fiction heuristic that we should have

$$
\left.\Phi_{\alpha \beta}[X ; q, t]\right|_{\substack{t=1 \\ q=1}}=S_{2} S_{1}^{n-2} .
$$

In particular for all such pairs we should have

$$
\operatorname{dim} \mathbf{M}_{\alpha} \cap \mathbf{M}_{\beta}=\frac{n !}{2} .
$$

It may be shown that this identity implies the $n$ !-conjecture.

In the same vein, by combining 3.10 with 3.11 , we derive that the symmetric function

$$
\Psi_{\alpha \beta}[X ; q, t]=-\nabla \Phi_{\alpha \beta}[X ; q, t]=T_{\alpha} T_{\beta} \frac{\tilde{H}_{\beta}[X ; q, t]-\tilde{H}_{\alpha}[X ; q, t]}{T_{\beta}-T_{\alpha}}
$$

must also be Schur positive since

$$
\frac{\Psi_{\alpha \beta}[X ; q, t]}{T_{\beta}} \quad \text { and } \quad \frac{\Psi_{\alpha \beta}[X ; q, t]}{T_{\beta}}
$$

should respectively give the Frobenius characteristics of the two $S_{n}$-modules

$$
\mathbf{M}_{\alpha} \cap\left(\mathbf{M}_{\alpha} \cap \mathbf{M}_{\beta}\right)^{\perp} \quad \text { and } \quad \mathbf{M}_{\beta} \cap\left(\mathbf{M}_{\alpha} \cap \mathbf{M}_{\beta}\right)^{\perp} \text {. }
$$

Further refinements of these Schur positivity conjectures may be obtained by means of the general forms of 3.10 and 3.11. But even the latter are but very special cases of the general positivity conjectures that are formulated in [2]. To keep our presentation to a reasonable size we shall only mention one example that we find most interesting. Namely, that with $\mu \vdash n+1$, and $\mathcal{P} \operatorname{red}(\mu)$ as given in 3.8, and the corner weights as defined above, the symmetric function

$$
\Xi_{\mu}=\sum_{s=1}^{m} \frac{\prod_{r=1}^{d-1}\left(x_{s}-u_{r}\right)}{\prod_{r=1 \quad r \neq s}^{d}\left(x_{s}-x_{r}\right)} \tilde{H}_{\nu^{(s)}} .
$$

should always be the Frobenius characteristic of a bigraded left regular representation of $S_{n}$. The reader is referred to [2] and [3] for further examples and the arguments justifying all these assertions.

Experimenting with our operators $\nabla, D_{k}$ and $D_{k}^{*}$ we quickly run into polynomials in $q, t$ with positive integer coefficients. Although we may usually find a representation theoretical "reason" for this, the actual proof invariably eludes us. In most cases the best we can do is derive the result from the positivity of the $q, t$-Kostka. The following result is a good instance in point.

THEOREM 3.1. On the Macdonald positivity conjecture, for any integer $k \geq 1$, we have:

$$
\left(\left(\frac{1+D_{1}^{*}}{M}\right)^{k} \mathbf{1}\right)\left[D_{\mu}(q, t)\right] \in \begin{cases}\mathrm{N}[q, t] & \text { if }|\mu| \geq k \\ \{0\} & \text { otherwise }\end{cases}
$$


Proof. From I.12 (iii)* we derive that

$$
\nabla\left(1+D_{1}^{*}\right)^{k} \nabla^{-1}=\left(1+e_{1}\right)^{k}
$$

and since $\nabla^{-1} \mathbf{1}=\mathbf{1}$ we may write

$$
\nabla\left(1+D_{1}^{*}\right)^{k} \mathbf{1}=\left(1+e_{1}\right)^{k} .
$$

Note further that from the definition of $\epsilon$ it follows that

$$
\mathcal{T}_{\epsilon}^{-1} e_{1}=e_{1}[X-\epsilon]=1+e_{1}
$$

and 3.29 can be converted to

$$
\nabla\left(1+D_{1}^{*}\right)^{k} \mathbf{1}=\mathcal{T}_{\epsilon}^{-1} e_{1}^{k}
$$

or, better yet

$$
\left(1+D_{1}^{*}\right)^{k} \mathbf{1}=\nabla^{-1} \mathcal{T}_{\epsilon}^{-1} e_{1}^{k}
$$

In view of I.16 we may rewrite this as

$$
\left(1+D_{1}^{*}\right)^{k} \mathbf{1}=\Pi e_{1}^{k}
$$

so we can use the identity in I.20 and obtain

$$
\left(\left(\frac{1+D_{1}^{*}}{M}\right)^{k} \mathbf{1}\right)\left[D_{\mu}(q, t)\right]= \begin{cases}0 & \text { if }|\mu|<k \\ \left\langle e_{n-k}^{*} e_{1}^{* k}, \tilde{H}_{\mu}\right\rangle_{*} & \text { if }|\mu|=n \geq k .\end{cases}
$$

This establishes the result for $|\mu|<k$. Now for $n \geq k$ the identity in 1.4 yields that

$$
\left(\left(\frac{1+D_{1}^{*}}{M}\right)^{k} \mathbf{1}\right)\left[D_{\mu}(q, t)\right]=\left\langle h_{n-k} h_{1}{ }^{k}, \tilde{H}_{\mu}\right\rangle=\delta_{1}^{k} \partial_{S_{n-k}} \tilde{H}_{\mu}
$$

Recalling that we have the expansion

$$
\partial_{S_{n-k}} S_{\lambda}[X]=\sum_{\lambda / \nu \in \mathcal{H}_{n-k}} S_{\nu}[X]
$$

where the symbol " $\lambda / \nu \in \mathcal{H}_{n-k}$ " is to denote that $\lambda / \nu$ is a horizontal $(n-k)$-strip, we see that substituting I.7 in 3.30 gives (for $\mu \vdash n \geq k$ )

$$
\begin{aligned}
\left(\left(\frac{1+D_{1}^{*}}{M}\right)^{k} \mathbf{1}\right)\left[D_{\mu}(q, t)\right] & =\delta_{1}^{k} \sum_{\lambda} \tilde{K}_{\lambda \mu}(q, t) \sum_{\lambda / \nu \in \mathcal{H}_{n-k}} S_{\nu}[X] \\
& =\sum_{\lambda} \tilde{K}_{\lambda \mu}(q, t) \sum_{\lambda / \nu \in \mathcal{H}_{n-k}} f_{\nu}
\end{aligned}
$$

where $f_{\nu}$ denotes the number of standard tableaux of shape $\nu$. This completes our proof. 
REMARK 3.1. We should point out that for $q=t=1$ the polynomial on the left hand side of 3.28 reduces to a very familiar number. In fact, since $\left.\tilde{H}_{\mu}[X ; q, t]\right|_{t=q=1} \dot{=}$ $e_{1}^{n}$, from 3.30 we derive that for $n \geq k$

$$
\left.\left(\left(\frac{1+D_{1}^{*}}{M}\right)^{k} \mathbf{1}\right)\left[D_{\mu}(q, t)\right]\right|_{\substack{t=1 \\ q=1}}=\partial_{S_{n-k}} \delta_{1}^{k} e_{1}^{n}=n(n-1) \cdots(n-k+1)
$$

We may thus view this polynomial as a $q, t$-analogue of the descending factorial. It would be interesting to find a combinatorial interpretation for this. The identity in 3.31 reveals that this should be a challenging task. Here, we can only suggest a very interesting representation theoretical interpretation.

Recall that if $\mathrm{M}$ is a bigraded $S_{n}$-module and $\mathcal{H}_{r s}(\mathrm{M})$ is the subspace consisting of the elements of $\mathbf{M}$ which are of bidegree $(r, s)$ then the "bigraded" Hilbert series $F_{\mathbf{M}}(q, t)$ of $\mathbf{M}$ is given by the formal sum

$$
F_{\mathbf{M}}(q, t)=\sum_{r, s \geq 0} t^{r} q^{s} \operatorname{dim} \mathcal{H}_{r s}(\mathbf{M})
$$

It is well known and easy to show that if $\Phi[X ; q, t]$ is the Frobenius characteristic of $\mathbf{M}$ then $F_{\mathbf{M}}(q, t)$ may be computed by means of the formula

$$
F_{\mathrm{M}}(q, t)=\partial_{1}^{n} \Phi[X ; q, t] .
$$

Now for $k \geq 1$ let

$$
\mathbf{M}_{\mu}^{(k)}=\mathcal{L}\left[\partial_{x_{1}}^{p_{1}} \partial_{y_{1}}^{q_{1}} \cdots \partial_{x_{k}}^{p_{k}} \partial_{y_{k}}^{q_{k}} \Delta_{\mu}\right]
$$

be the linear span of all polynomials obtained by differentiating $\Delta_{\mu}(x ; y)$ in all possible ways but only with respect to the first $k$ of the $x_{i}$ and $y_{i}$. For convenience let $F_{\mu}^{(k)}(q, t)$ denote the Hilbert series of $\mathbf{M}_{\mu}^{(k)}$ and set

$$
G_{\mu}^{(k)}(q, t)=T_{\mu} F_{\mu}^{(k)}(1 / q, 1 / t) .
$$

It develops that the polynomial in 3.28 should be none other than the Hilbert series of $\mathbf{M}_{\mu}^{(k)}$. More precisely

Theorem 3.2. On the $n$ !-conjecture, for $|\mu| \geq k$ we have

$$
\left(\left(\frac{1+D_{1}^{*}}{M}\right)^{k} \mathbf{1}\right)\left[D_{\mu}(q, t)\right]=G_{\mu}^{(k)}(q, t) .
$$

Proof. In view of 3.30 we need only show that

$$
G_{\mu}^{(k)}(q, t)=\delta_{1}^{k} \partial_{S_{n-k}} \tilde{H}_{\mu} .
$$

To this end, we start by noting that the space $\mathbf{M}_{\mu}^{(k)}$ may also be obtained by antisymmetrizing all the elements of $\mathbf{M}_{\mu}$ with respect to the symmetric group $S_{[k+1, \ldots, n]}$. More precisely, let $B=[k+1, \ldots, n]$ and, using Young's notation, let $P[B]$ and $N[B]$ respectively denote, the formal sums of all the elements and all the "signed" elements of $S_{[k+1, \ldots, n]}$. Our claim is that

$$
\mathbf{M}_{\mu}^{(k)}=N[B] \mathbf{M}_{\mu} .
$$


To see this note that since we can factorize any monomial $m(x, y)=$ $m\left(x_{1}, y_{1}, \ldots, x_{n}, y_{n}\right)$ in the form

$$
m=m_{1}\left(x_{1}, y_{1}, \ldots, x_{k}, y_{k}\right) \times m_{2}\left(x_{k+1}, y_{k+1}, \ldots, x_{k}, y_{k}\right)
$$

we shall have

$$
N[B]\left(m\left(\partial_{x} ; \partial_{y}\right) \Delta_{\mu}(x ; y)\right)=m_{1}\left(\partial_{x} ; \partial_{y}\right)\left(P[B] m_{2}\right)\left(\partial_{x} ; \partial_{y}\right) \Delta_{\mu}(x ; y) .
$$

Since the polynomial $P[B] m_{2}\left(x_{k+1}, y_{k+1}, \ldots, x_{k}, y_{k}\right)$ is invariant under the diagonal action of $S_{[k+1, \ldots, n]}$, it follows from a theorem of H. Weyl (see [24]) that we can express it as a polynomial in the polarized power sums

$$
\sum_{i=k+1}^{n} x_{i}^{r} y_{i}^{s}
$$

However, starting from the definition in 3.2 we can easily show that for $r+s>0$ we have

$$
\sum_{i=1}^{n} \partial_{x_{i}}^{r} \partial_{y_{i}}^{s} \Delta_{\mu}(x ; y)=0 \text {. }
$$

In other words

$$
\sum_{i=k+1}^{n} \partial_{x_{i}}^{r} \partial_{y_{i}}^{s} \Delta_{\mu}(x ; y)=-\sum_{i=1}^{k} \partial_{x_{i}}^{r} \partial_{y_{i}}^{s} \Delta_{\mu}(x ; y)
$$

This given it follows that for some polynomial $Q_{m_{2}}\left(x_{1}, y_{1}, \ldots, x_{k}, y_{k}\right)$ we have (3.41)

$\left(P[B] m_{2}\right)\left(\partial_{x_{k+1}}, \partial_{y_{k+1}}, \ldots, \partial_{x_{n}}, \partial_{y_{n}}\right) \Delta_{\mu}(x ; y)=Q_{m_{2}}\left(\partial_{x_{1}}, \partial_{y_{1}}, \ldots, \partial_{x_{k}}, \partial_{y_{k}}\right) \Delta_{\mu}(x ; y)$.

Substituting 3.41 in 3.40 gives

$$
\begin{aligned}
& N[B]\left(m\left(\partial_{x} ; \partial_{y}\right) \Delta_{\mu}(x ; y)\right) \\
& \quad=m_{1}\left(\partial_{x_{1}}, \partial_{y_{1}}, \ldots, \partial_{x_{k}}, \partial_{y_{k}}\right) Q_{m_{2}}\left(\partial_{x_{1}}, \partial_{y_{1}}, \ldots, \partial_{x_{k}}, \partial_{y_{k}}\right) \Delta_{\mu}(x ; y) .
\end{aligned}
$$

This proves that

$$
N[B] \mathbf{M}_{\mu} \subseteq \mathbf{M}_{\mu}^{(k)}
$$

On the other hand, for any monomial $m_{1}\left(x_{1}, y_{1}, \ldots, x_{k}, y_{k}\right)$, we clearly have

$$
m_{1}\left(\partial_{x_{1}}, \partial_{y_{1}}, \ldots, \partial_{x_{k}}\right) \Delta_{\mu}(x ; y)=\frac{1}{k !} N[B]\left(m_{1}\left(\partial_{x_{1}}, \partial_{y_{1}}, \ldots, \partial_{x_{k}}\right) \Delta_{\mu}(x ; y)\right)
$$

Thus the reverse inclusion

$$
\mathbf{M}_{\mu}^{(k)} \subseteq N[B] \mathbf{M}_{\mu}
$$

is trivial, proving 3.39 .

Now it may be shown, (see [10] Proposition 6.1) that if $\mathbf{M}$ is a bigraded $S_{n}$-module with bivariate Frobenius characteristic $\Phi_{\mathbf{M}}(X ; q, t)$, then the bivariate Frobenius characteristic of $N[B] \mathrm{M}$, as an $S_{[1, \ldots, k]}$-module is given by the simple expression

$$
\partial_{S_{1^{n-k}}} \Phi_{\mathbf{M}}(X ; q, t) \text {. }
$$

In particular, the Hilbert series of $N[B] \mathrm{M}$, may be computed from the formula

$$
F_{N[B] \mathrm{M}}(q, t)=\delta_{1}^{k} \partial_{S_{1^{n-k}}} \Phi_{\mathrm{M}}(X ; q, t) .
$$


Applying this result to the case $\mathbf{M}=\mathbf{M}_{\mu}$, we see that, on the $n !$-conjecture, we must have

$$
F_{\mu}^{(k)}(q, t)=F_{\mathbf{M}_{\mu}^{(k)}}(q, t)=F_{N[B] \mathbf{M}_{\mu}}(q, t)=\delta_{1}^{k} \partial_{S_{1^{n-k}}} \tilde{H}_{\mu}(X ; q, t) .
$$

Finally, we observe that 1.13 gives

$$
\tilde{H}_{\mu}[X ; 1 / q, 1 / t]=\frac{1}{T_{\mu}} \omega \tilde{H}_{\mu}[X ; q, t] .
$$

Thus combining 3.36 and 3.43 we derive that

$$
\begin{aligned}
G_{\mu}^{(k)}(q, t) & =T_{\mu} \delta_{1}^{k} \partial_{S_{1^{n-k}}} \tilde{H}_{\mu}[X ; 1 / q, 1 / t] \\
& =T_{\mu} \delta_{1}^{k} \partial_{S_{1^{n-k}}} \frac{1}{T_{\mu}} \omega \tilde{H}_{\mu}[X ; q, t] \\
& =\delta_{1}^{k} \partial_{S_{n-k}} \tilde{H}_{\mu}[X ; q, t]
\end{aligned}
$$

This proves 3.38, completing our argument.

We should note that both $G_{\mu}^{(k)}(q, t)$ and $F_{\mu}^{(k)}(q, t)$ satisfy the same recursion. More precisely,

THEOREM 3.3. For $|\mu| \geq k \geq 1$ we have

$$
\begin{aligned}
\text { a) } G_{\mu}^{(k)}(q, t) & =\sum_{\nu \rightarrow \mu} c_{\mu, \nu}(q, t) G_{\nu}^{(k-1)}(q, t), \\
\text { b) } F_{\mu}^{(k)}(q, t) & =\sum_{\nu \rightarrow \mu} c_{\mu, \nu}(q, t) F_{\nu}^{(k-1)}(q, t) .
\end{aligned}
$$

with the initial conditions

$$
\begin{aligned}
\text { a) } G_{\mu}^{(1)}(q, t) & =B_{\mu}(q, t) \\
\text { b) } F_{\mu}^{(1)}(q, t) & =T_{\mu} B_{\mu}(1 / q, 1 / t) .
\end{aligned}
$$

Proof. Note that from 3.43 and 1.36 we immediately get that for $k \geq 1$

$$
\begin{aligned}
F_{\mu}^{(k)}(q, t) & =\delta_{1}^{k-1} \partial_{S_{1^{n-k}}} \delta_{1} \tilde{H}_{\mu}(X ; q, t) \\
& =\sum_{\nu \rightarrow \mu} c_{\mu \nu}(q, t) \delta_{1}^{k-1} \partial_{S_{1^{n-k}}} \tilde{H}_{\nu}(X ; q, t) .
\end{aligned}
$$

This proves $3.44 \mathrm{~b}$ ). The recursion in $3.44 \mathrm{a}$ ) is proved in exactly the same way by means of 3.38 . Note next that 3.37 gives

$$
G_{\mu}^{(1)}(q, t)=\left(\frac{1+D_{1}^{*}}{M} \mathbf{1}\right)\left[D_{\mu}(q, t)\right] .
$$

Now from the definition in I.10 we immediately get $D_{1}^{*} 1=e_{1}$. Thus, recalling I.4, we obtain

$$
G_{\mu}^{(1)}(q, t)=\frac{1+e_{1}}{M}\left[D_{\mu}(q, t)\right]=\frac{1+e_{1}}{M}\left[M B_{\mu}(q, t)-1\right]=B_{\mu}(q, t),
$$

as desired. The identity in $3.45 \mathrm{~b}$ ) follows from 3.36 . 
REMARK 3.2. It would seem that we should be able to construct a proof of the positivity of the polynomial $G_{\mu}^{(k)}(q, t)$ by an induction argument based on the recursion in 3.44. In fact, we shall see that can do this for $k=2$. The question remains whether a similar argument can be carried out in full generality.

Recalling the definitions of corners weights given in $3.16,3.17$ and 3.18 let us denote by $B_{i j}(q, t)$ the portion of the polynomial

$$
B_{\mu}(q, t)=\sum_{s \in \mu} t^{l_{\mu}^{\prime}(s)} q^{a_{\mu}^{\prime}(s)}
$$

that is contributed by the rectangle

$$
\mathcal{R}_{i j}=\left\{\left(l^{\prime}, a^{\prime}\right): l_{j+1}^{\prime}<l^{\prime} \leq l_{j}, a_{i-1}^{\prime}<a^{\prime} \leq a_{i}^{\prime}\right\} .
$$

Note that we must have then

$$
\begin{aligned}
B_{i j}(q, t)=\sum_{\left(l^{\prime}, a^{\prime}\right) \in \mathcal{R}_{i j}} t^{l^{\prime}} q^{a^{\prime}} & =t^{l_{j+1}^{\prime}} q^{a_{i-1}^{\prime}}\left(t+\cdots+t^{l_{j}^{\prime}-l_{j+1}^{\prime}}\right)\left(q+\cdots+q^{a_{i}^{\prime}-a_{i-1}^{\prime}}\right) \\
& =t^{l_{j+1}^{\prime}} q^{a_{i-1}^{\prime}} \frac{\left(1-t^{l_{j}^{\prime}-l_{j+1}^{\prime}}\right)}{1-1 / t} \frac{\left(1-q^{a_{i}^{\prime}-a_{i-1}^{\prime}}\right)}{1-1 / q} \\
& =t^{l_{j+1}^{\prime}} q^{a_{i-1}^{\prime}} \frac{\left(1-x_{j} / u_{j}\right)\left(1-x_{i} / u_{i-1}\right)}{\tilde{M}}
\end{aligned}
$$

Next call $\mathcal{R}_{i j}^{*}$ the rectangle obtained by lifting $\mathcal{R}_{i j}$ so that its top side lies on the boundary of the Ferrers diagram of $\mu$. We can easily see that we must have

$$
B_{i j}^{*}(q, t)=\sum_{s \in \mathcal{R}_{i j}^{*}} t^{l^{\prime}} q^{a^{\prime}}=t^{l_{i}^{\prime}-l_{j}^{\prime}} B_{i j}(q, t) .
$$

This given, combining with 3.46 and using the definitions in 3.16 and 3.17 we obtain that

$$
B_{i j}^{*}(q, t)=\frac{t q}{x_{j} M}\left(u_{j}-x_{j}\right)\left(u_{i-1}-x_{i}\right)
$$

Now note that for a given $1 \leq j \leq d$ the rectangles $\mathcal{R}_{i j}^{*}$ are disjoint as $i$ varies from 1 to $j$. Thus

$$
B_{\mu}(q, t)-\sum_{i=1}^{j} B_{i j}^{*}(q, t)>>0
$$

where the symbol " $A>>B$ " is to mean that the difference $A-B$ has non-negative integer coefficients. In particular we derive that

$$
B_{\mu}^{2}-\sum_{j=1}^{d} x_{j} \sum_{i=1}^{j} B_{i j}^{*}(q, t)>B_{\mu}^{2}-B_{\mu} \sum_{j=1}^{d} x_{j}>>0 .
$$

Taking this into account we see from 3.47 that the positivity of $G_{\mu}^{(2)}(q, t)$ is an immediate consequence of the following explicit formula . 
THEOREM 3.4 .

$$
G_{\mu}^{(2)}(q, t)=B_{\mu}^{2}-\frac{t q}{M} \sum_{j=1}^{d} \sum_{i=1}^{j}\left(x_{i}-u_{i-1}\right)\left(x_{j}-u_{j}\right)
$$

Proof. Combining the recursion in 3.44 a) with the initial condition in 3.45 a) we get that

$$
G_{\mu}^{(2)}(q, t)=\sum_{\nu \rightarrow \mu} c_{\mu \nu}(q, t) B_{\nu}(q, t)
$$

Now since $B_{\nu}=B_{\mu}-T_{\mu} / T_{\nu}$ we may rewrite this as

$$
G_{\mu}^{(2)}(q, t)=\sum_{\nu \rightarrow \mu} c_{\mu \nu}(q, t) B_{\mu}(q, t)-\sum_{\nu \rightarrow \mu} c_{\mu \nu}(q, t) T_{\mu} / T_{\nu}
$$

But then 1.37 gives

$$
G_{\mu}^{(2)}(q, t)=B_{\mu}^{2}(q, t)-\frac{t q}{M} h_{2}\left[D_{\mu}(q, t) / q t\right] .
$$

Now it is shown in [15] (Proposition 2.3) that

$$
D_{\mu}(q, t) / q t=x_{1}+\cdots+x_{d}-u_{0}-\cdots-u_{d}
$$

This given, 3.48 follows from the simple symmetric function identity

$$
h_{2}\left[x_{1}+\cdots+x_{d}-u_{0}-\cdots-u_{d}\right]=\sum_{i=1}^{d} \sum_{j=i}^{d}\left(x_{i}-u_{i-1}\right)\left(x_{j}-u_{j}\right) .
$$

We terminate this section with three remarkable $\nabla$-positivity conjectures.

Conjecture II. For any partitions $\lambda, \mu$ we have

$$
(-1)^{|\mu|-l(\mu)}\left\langle\nabla \tilde{H}_{\mu}(x ; 0, t), S_{\lambda}(x)\right\rangle \in \mathbf{N}[q, t] .
$$

REMARK 3.3. The fact that $\nabla \tilde{H}_{\mu}(x ; 0, t)$ appears to be virtually positive was discovered by computer experimentation by A. Lascoux, after learning of the virtual positivity of $\nabla S_{\lambda}$. However, the identification of the sign must be as stated in 3.49 to be consistent with Conjecture I. To see this note that since we have

$$
\tilde{H}_{\mu}(x ; 0, t)=\sum_{\lambda} S_{\lambda}(x) \tilde{K}_{\lambda \mu}(t)
$$

with $\tilde{K}_{\lambda \mu}(t)$ the Kostka-Foulkes polynomials, it follows that for $\mu=\left(\mu_{1}, \mu_{2}, \ldots, \mu_{k}\right)$

$$
\tilde{H}_{\mu}(x ; 0,1)=\sum_{\lambda} S_{\lambda}(x) K_{\lambda \mu}=h_{\mu_{1}} h_{\mu_{2}} \cdots h_{\mu_{k}}
$$

with $K_{\lambda \mu}$ denoting the ordinary Kostka number. This given, from the multiplicativity of $\left.\nabla\right|_{t=1}$ we immediately derive (using the notation of section 2)

$$
\left.\nabla \tilde{H}_{\mu}(x ; 0, t)\right|_{t \rightarrow 1}=\tilde{\nabla} h_{\mu_{1}} \tilde{\nabla} h_{\mu_{2}} \cdots \tilde{\nabla} h_{\mu_{k}} .
$$


However, setting $t \rightarrow 1, m \rightarrow 1$ and $\lambda \rightarrow(m)$ in I.8 we derive that, on the validity of Conjecture I, the expression $(-1)^{\iota\left(1^{m}\right)} \tilde{\nabla} h_{m}$ must, for all $m$, evaluate to a Schurpositive symmetric function. However, we see that

$$
(-1)^{\iota\left(1^{m}\right)}=\left(\begin{array}{c}
m \\
2
\end{array}\right)+\sum_{1<i-1}(i-1-1)=1+2+\cdots+m-1+0+1+\cdots m-2 \equiv m-1(\bmod 2) .
$$

Using this in 3.50 yields that, according to Conjecture I, the symmetric function

$$
(-1)^{\mu_{1}+\cdots+\mu_{k}-k} \tilde{\nabla} h_{\mu_{1}} \tilde{\nabla} h_{\mu_{2}} \cdots \tilde{\nabla} h_{\mu_{k}}=\left.(-1)^{|\mu|-l(\mu)} \nabla \tilde{H}_{\mu}(x ; 0, t)\right|_{t \rightarrow 1}
$$

must be Schur positive. This forces the choice of sign in 3.49.

Our next conjecture is truly surprising and fraught with tantalizing combinatorial implications.

Conjecture III. For every partition $\mu$ the symmetric function

$$
\nabla \omega \tilde{H}_{\mu}(x ; 0,1 / t)
$$

is integral in $q$ and $t$ and Schur positive. Moreover, for any pair of partitions $\nu$ and $\mu$ with $\mu>\nu$ in dominance, the difference

$$
\nabla \omega \tilde{H}_{\mu}(x ; 0,1 / t)-\nabla \omega \tilde{H}_{\nu}(x ; 0,1 / t)
$$

is always Schur positive as well.

REMARK 3.4. We should note that for $\mu=\left(\mu_{1}, \mu_{2}, \ldots, \mu_{k}\right)$ we have the specialization

$$
\begin{aligned}
\left.\nabla \omega \tilde{H}_{\mu}(x ; 0,1 / t)\right|_{t \rightarrow 1} & =\tilde{\nabla} e_{\mu_{1}} \tilde{\nabla} e_{\mu_{2}} \cdots \tilde{\nabla} e_{\mu_{k}} \\
& =D H_{\mu_{1}}(x ; q, 1) D H_{\mu_{2}}(x ; q, 1) \cdots D H_{\mu_{k}}(x ; q, 1) .
\end{aligned}
$$

It develops that with this specialization, it is not difficult to show that the difference

$$
\left.\nabla \omega \tilde{H}_{\mu}(x ; 0,1 / t)\right|_{t \rightarrow 1}-\left.\nabla \omega \tilde{H}_{\nu}(x ; 0,1 / t)\right|_{t \rightarrow 1}
$$

is always Schur positive. Neveretheless, it should make quite an interesting and challenging research problem to give a combinatorial proof of this positivity based on the Parking Function interpretation of $D H_{m}(x ; q, 1)$ described in [11].

Surprisingly even the monomial symmetric functions have virtually Schur positive $\nabla$-images. More precisely

CONJECTURE IV. For any pair of partitions $\lambda, \mu \vdash n$

$$
(-1)^{n-l(\mu)}\left\langle\nabla m_{\mu}, S_{\lambda}\right\rangle \in \mathbf{N}[q, t] .
$$

Moreover these polynomials have coefficients that are doubly unimodal in $q$ and $t$.

For the benefit of the reader we have displayed in the following page the Schur function expansions of the polynomials $\nabla \omega \tilde{H}_{\mu}(x ; 0,1 / t)$ for all $\mu \vdash 4$. We can clearly observe from this display the monotonicity with respect to dominance as well as all sorts of triangularities. 


$$
\begin{aligned}
& \begin{array}{lllll}
S_{4} & S_{31} & S_{22} & S_{211} & S_{1111}
\end{array} \\
& \nabla \omega \tilde{H}_{(4)}(x ; 0,1 / t) \rightarrow[1]\left[\begin{array}{llll}
1 & 0 & 0 & 0 \\
1 & 1 & 0 & 0 \\
1 & 1 & 1 & 0 \\
1 & 1 & 1 & 0
\end{array}\right]\left[\begin{array}{lllll}
1 & 0 & 0 & 0 & 0 \\
0 & 1 & 0 & 0 & 0 \\
1 & 1 & 1 & 0 & 0 \\
0 & 1 & 1 & 1 & 0 \\
0 & 0 & 1 & 0 & 1
\end{array}\right]\left[\begin{array}{llllll}
1 & 0 & 0 & 0 & 0 & 0 \\
1 & 1 & 0 & 0 & 0 & 0 \\
1 & 2 & 1 & 0 & 0 & 0 \\
0 & 2 & 2 & 1 & 0 & 0 \\
0 & 1 & 2 & 2 & 1 & 0 \\
0 & 0 & 0 & 1 & 1 & 1
\end{array}\right]\left[\begin{array}{lllllll}
1 & 0 & 0 & 0 & 0 & 0 & 0 \\
0 & 1 & 0 & 0 & 0 & 0 & 0 \\
0 & 1 & 1 & 0 & 0 & 0 & 0 \\
0 & 1 & 1 & 1 & 0 & 0 & 0 \\
0 & 0 & 1 & 1 & 1 & 0 & 0 \\
0 & 0 & 0 & 1 & 1 & 1 & 0 \\
0 & 0 & 0 & 0 & 0 & 1 & 0
\end{array}\right] \\
& \nabla \omega \tilde{H}_{(3,1)}(x ; 0,1 / t) \rightarrow[1]\left[\begin{array}{lll}
1 & 0 & 0 \\
1 & 0 & 0 \\
1 & 1 & 0 \\
0 & 1 & 1
\end{array}\right] \quad\left[\begin{array}{lll}
1 & 0 & 0 \\
0 & 0 & 0 \\
1 & 1 & 0 \\
0 & 1 & 0 \\
0 & 0 & 1
\end{array}\right] \quad\left[\begin{array}{llll}
1 & 0 & 0 & 0 \\
1 & 0 & 0 & 0 \\
1 & 1 & 0 & 0 \\
0 & 2 & 0 & 0 \\
0 & 1 & 2 & 0 \\
0 & 0 & 0 & 1
\end{array}\right] \quad\left[\begin{array}{llll}
1 & 0 & 0 & 0 \\
0 & 0 & 0 & 0 \\
0 & 1 & 0 & 0 \\
0 & 1 & 0 & 0 \\
0 & 0 & 1 & 0 \\
0 & 0 & 0 & 1 \\
0 & 0 & 0 & 0
\end{array}\right] \\
& \nabla \omega \tilde{H}_{(2,2)}(x ; 0,1 / t) \rightarrow[1]\left[\begin{array}{ll}
1 & 0 \\
1 & 0 \\
1 & 1 \\
0 & 1
\end{array}\right] \quad\left[\begin{array}{lll}
1 & 0 & 0 \\
0 & 0 & 0 \\
1 & 1 & 0 \\
0 & 1 & 0 \\
0 & 0 & 1
\end{array}\right] \quad\left[\begin{array}{lll}
1 & 0 & 0 \\
1 & 0 & 0 \\
1 & 1 & 0 \\
0 & 2 & 0 \\
0 & 1 & 1 \\
0 & 0 & 0
\end{array}\right] \quad\left[\begin{array}{lll}
1 & 0 & 0 \\
0 & 0 & 0 \\
0 & 1 & 0 \\
0 & 1 & 0 \\
0 & 0 & 1 \\
0 & 0 & 0 \\
0 & 0 & 0
\end{array}\right] \\
& \left.\nabla \omega \tilde{H}_{(2,1,1)}(x ; 0,1 / t) \rightarrow[1]\left[\begin{array}{ll}
1 & 0 \\
1 & 0 \\
1 & 0 \\
0 & 1
\end{array}\right] \quad\left[\begin{array}{ll}
1 & 0 \\
0 & 0 \\
1 & 0 \\
0 & 1 \\
0 & 0
\end{array}\right] \quad\left[\begin{array}{ll}
1 & 0 \\
1 & 0 \\
1 & 0 \\
0 & 1 \\
0 & 1 \\
0 & 0
\end{array}\right]\right]\left[\begin{array}{ll}
1 & 0 \\
0 & 0 \\
0 & 0 \\
0 & 1 \\
0 & 0 \\
0 & 0 \\
0 & 0
\end{array}\right] \\
& \nabla \omega \tilde{H}_{(1,1,1,1)}(x ; 0,1 / t) \rightarrow[1] \quad\left[\begin{array}{l}
1 \\
1 \\
1 \\
0
\end{array}\right] \quad\left[\begin{array}{l}
1 \\
0 \\
1 \\
0 \\
0
\end{array}\right] \quad\left[\begin{array}{l}
1 \\
1 \\
1 \\
0 \\
0 \\
0
\end{array}\right] \quad\left[\begin{array}{l}
1 \\
0 \\
0 \\
0 \\
0 \\
0 \\
0
\end{array}\right]
\end{aligned}
$$

REMARK 3.5. We should note that our positivity conjectures can be used to deduce further ones through the use of $1.14 \mathrm{~b}$ ) and the self-adjointness of $\nabla$ with respect to the $*$-scalar product. This is easily seen from the proof of the following simple identities. 
Theorem 3.5. For $\lambda \vdash n$ set

$$
\nabla S_{\mu}=\sum_{\lambda \vdash n} S_{\lambda} \alpha_{\lambda, \mu}(q, t)
$$

then

$$
\begin{aligned}
\nabla S_{\mu}\left[\frac{X}{M}\right] & =\sum_{\lambda \vdash n} S_{\lambda}\left[\frac{X}{M}\right] \alpha_{\mu^{\prime}, \lambda^{\prime}}(q, t) \\
\nabla^{-1} S_{\mu} & =\sum_{\lambda \vdash n} S_{\lambda} \alpha_{\lambda^{\prime}, \mu^{\prime}}\left(\frac{1}{q}, \frac{1}{t}\right) \\
\nabla^{-1} S_{\mu}\left[\frac{X}{M}\right] & =\sum_{\lambda \vdash n} S_{\lambda}\left[\frac{X}{M}\right] \alpha_{\mu, \lambda}\left(\frac{1}{q}, \frac{1}{t}\right) \\
\alpha_{\lambda, \mu}(q, t) & =\alpha_{\lambda, \mu}(t, q) .
\end{aligned}
$$

Proof. It follows from $1.2 \mathrm{~b}$ ) that

$$
\alpha_{\lambda, \mu}(q, t)=\left\langle\nabla S_{\mu}, S_{\lambda^{\prime}}^{*}\right\rangle_{*}
$$

and $*$-self-adjointness of $\nabla$ gives

$$
\alpha_{\lambda, \mu}(q, t)=\left\langle S_{\mu}, \nabla S_{\lambda^{\prime}}^{*}\right\rangle_{*}=\left\langle\nabla S_{\lambda^{\prime}}^{*}, S_{\mu}\right\rangle_{*}
$$

and 3.52 follows immediately since, again from $1.2 \mathrm{~b}$ ), we have

$$
\nabla S_{\mu}^{*}=\sum_{\lambda \vdash n} S_{\lambda}^{*}\left\langle\nabla S_{\mu}^{*}, S_{\lambda^{\prime}}\right\rangle_{*}
$$

Note further that applying the operator " $\downarrow$ " to both sides of 3.51 and using $1.14 \mathrm{~b}$ ) gives

$$
\nabla^{-1} S_{\mu^{\prime}}=\sum_{\nu \vdash n} S_{\lambda^{\prime}} \alpha_{\lambda, \mu}\left(\frac{1}{q}, \frac{1}{t}\right)
$$

and 3.53 immediately follows by the replacements $\mu \rightarrow \mu^{\prime}$ and $\lambda \rightarrow \lambda^{\prime}$. The identity in 3.54 can then be deduced from 3.53 in the same way we deduced 3.52 from 3.51 .

Finally it is shown in [9] that we have

$$
\tilde{H}_{\mu}[X ; t, q]=\tilde{H}_{\mu^{\prime}}[X ; q, t] .
$$

Note further that from the definition of $T_{\mu}$ in I.4 we get that

$$
\left.T_{\mu}\right|_{t \leftrightarrow q}=T_{\mu^{\prime}}
$$

where the symbol " $\left.\right|_{t \leftrightarrow q}$ " is to represent the operation of interchanging $t$ and $q$ in the preceding expression. This given, from I.5 we immediately derive that

$$
\left(\left.\nabla\right|_{t \leftrightarrow q}\right) \tilde{H}_{\mu}=\left(\left.\nabla\right|_{t \leftrightarrow q}\right)\left(\left.\tilde{H}_{\mu^{\prime}}\right|_{t \leftrightarrow q}\right)=\left.\nabla \tilde{H}_{\mu^{\prime}}\right|_{t \leftrightarrow q}=\left.T_{\mu^{\prime}} \tilde{H}_{\mu^{\prime}}\right|_{t \leftrightarrow q}=T_{\mu} \tilde{H}_{\mu} .
$$

In other words we have

$$
\left.\nabla\right|_{t \leftrightarrow q}=\nabla
$$


and this is equivalent to the validity of the identities in 3.55 for all $\lambda$ and $\mu$.

In the same vein we can easily see that Conjecture III may be restated in the following equivalent form.

ConjeCture III'. For every pair of partitions $\mu, \lambda$ we have

$$
\left.\nabla^{-1} \tilde{H}_{\mu}(x ; 0, t)\right|_{S_{\lambda}} \in \mathbf{N}\left[\frac{1}{q}, \frac{1}{t}\right]
$$

Moreover, for any pair of partitions $\nu$ and $\mu$ with $\mu>\nu$ in dominance, we have

$$
\nabla^{-1} \tilde{H}_{\mu}(x ; 0, t)-\left.\nabla^{-1} \tilde{H}_{\nu}(x ; 0, t)\right|_{S_{\lambda}} \in \mathbf{N}\left[\frac{1}{q}, \frac{1}{t}\right]
$$

for all $\lambda$.

The stated equivalence is another consequence of $1.14 \mathrm{~b}$ ) which gives that

$$
\downarrow \nabla \omega \tilde{H}_{\mu}(x ; 0,1 / t)=\nabla^{-1} \tilde{H}_{\mu}(x ; 0, t)
$$

REMARK 3.6. In the case $\mu=\left(2,1^{n-2}\right)$ we can give a representation theoretical proof of the Schur positivity of the polynomial $\nabla \omega \tilde{H}_{\mu}[X ; 0,1 / t]$. Let us recall that if

$$
\left\{\nu_{1}, \nu_{2}, \ldots, \nu_{d},\right\}
$$

are the predecessors of a given partition $\mu$, then $\phi_{\mu}[X ; q, t]$ denotes the Frobenius characteristic of the intersection

$$
\bigcap_{i=1}^{d} \mathbf{M}_{\nu_{i}}
$$

Now, using the "Science fiction" heuristic, it is shown in ( [3] Theorem 3.2) that the Frobenius characteristic of the sum

$$
\sum_{i=1}^{d} \mathbf{M}_{\nu_{i}}
$$

is given by the polynomial

$$
\frac{(-1)^{d-1} \nabla^{d} \phi_{\mu}[X ; q, t]}{T_{\nu_{1}} T_{\nu_{2}} \cdots T_{\nu_{d}}} .
$$

In the case of a partition $\mu$ with two predecessors $\alpha, \beta$ this result reduces to (see 3.10 and 3.22)

$$
\text { Fch } \mathbf{M}_{\alpha}+\mathbf{M}_{\beta}=-\frac{1}{T_{\alpha} T_{\beta}} \nabla^{2}\left(\frac{T_{\beta} \tilde{H}_{\alpha}-T_{\alpha} \tilde{H}_{\beta}}{T_{\beta}-T_{\alpha}}\right)=\nabla \frac{\tilde{H}_{\alpha}-\tilde{H}_{\beta}}{T_{\alpha}-T_{\beta}} .
$$

Using 1.14, this relation may also be rewritten as

$$
F \operatorname{ch} \mathbf{M}_{\alpha}+\mathbf{M}_{\beta}=\nabla \downarrow \frac{\tilde{H}_{\alpha} / T_{\alpha}-\tilde{H}_{\beta} / T_{\beta}}{1 / T_{\alpha}-1 / T_{\beta}}=\nabla \downarrow \frac{T_{\beta} \tilde{H}_{\alpha}-T_{\alpha} \tilde{H}_{\beta}}{T_{\beta}-T_{\alpha}} .
$$

In summary we see (again from 3.11) that in the two corner case we must have

$$
F c h \mathbf{M}_{\alpha}+\mathbf{M}_{\beta}=\nabla \downarrow F c h \mathbf{M}_{\alpha} \cap \mathbf{M}_{\beta} .
$$


It has been shown in [2] that all of the assertions of the Science fiction heuristics do hold true when $\mu$ is a hook. Note further that, in the particular case $\mu=\left(2,1^{n+1}\right)$, $\alpha=\left(1^{n}\right)$ and $\beta=\left(2,1^{n-2}\right)$ we must have

$$
F c h \mathbf{M}_{\alpha} \cap \mathbf{M}_{\beta}=\tilde{H}_{21^{n-2}}[X ; 0, t] .
$$

To see this, observe that from 3.15 we get the equations

$$
\text { a) } \tilde{H}_{\alpha}=\phi^{(2)}+\frac{\phi^{(1)}}{T_{\beta}} \quad, \quad \text { b) } \tilde{H}_{\beta}=\phi^{(2)}+\frac{\phi^{(1)}}{T_{\alpha}} \text {. }
$$

Now in this case

$$
\tilde{H}_{\alpha}=\tilde{H}_{1^{n}}=h_{n}\left[\frac{X}{1-t}\right](1-t)\left(1-t^{2}\right) \cdots\left(1-t^{n}\right) .
$$

Moreover, since the polynomials in $\mathbf{M}_{1^{n}}$ have no $y_{i}{ }^{\prime} s$, the Frobenius characteristic of $\mathbf{M}_{\alpha} \cap \mathbf{M}_{\beta}$, namely $\phi^{(2)}$, can have no $q^{\prime} s$. Thus, combining this observation with 3.60 and the fact that $T_{\beta}=T_{21^{n-2}}=q t^{\left(\begin{array}{c}n-1 \\ 2\end{array}\right)}$, we derive from 3.59 a) that $\phi^{(1)}$ must have $q$ as a factor. This given, by letting $q \rightarrow 0$ in $3.59 \mathrm{~b}$ ) we finally get that

$$
\phi^{(2)}=\tilde{H}_{\beta}[X ; 0, t] \text {, }
$$

and this is 3.58. Substituting this result in 3.57 yields

$$
F \operatorname{ch~} \mathbf{M}_{\alpha}+\mathbf{M}_{\beta}=\nabla \downarrow \tilde{H}_{\beta}[X ; 0, t]=\nabla \omega \tilde{H}_{21^{n-2}}[X ; 0,1 / t] .
$$

This establishes the Schur positivity of $\nabla \omega \tilde{H}_{21^{n-2}}[X ; 0,1 / t]$. Incidentally, this also shows the Schur-positivity of the difference

$$
\nabla \omega \tilde{H}_{\mu}(x ; 0,1 / t)-\nabla \omega \tilde{H}_{\nu}(x ; 0,1 / t)
$$

for $\mu=\left(2,1^{n-2}\right)$ and $\nu=\left(1^{n}\right)$.

4. The $q, t$-Catalan revisited. We recall that in [11] Garsia-Haiman conjecture that the symmetric function

$$
D H_{n}[X ; q, t]=\sum_{\mu \vdash n} \frac{T_{\mu} \tilde{H}_{\mu}[X ; q, t] M B_{\mu}(q, t) \Pi_{\mu}(q, t)}{\tilde{h}_{\mu}(q, t) \tilde{h}_{\mu}^{\prime}(q, t)}
$$

should give the Frobenius characteristic of the Diagonal Harmonic alternants in the variables $x_{1}, \ldots, x_{n} ; y_{1}, \ldots, y_{n}$. Since it can be shown (see [12]) that

$$
\left.\tilde{H}_{\mu}[X ; q, t]\right|_{S_{1^{n}}}=T_{\mu}
$$

we see that the rational expression

$$
C_{n}(q, t)=\left.D H_{n}[X ; q, t]\right|_{S_{1^{n}}}=\sum_{\mu \vdash n} \frac{T_{\mu}^{2} M B_{\mu}(q, t) \Pi_{\mu}(q, t)}{\tilde{h}_{\mu}(q, t) \tilde{h}_{\mu}^{\prime}(q, t)},
$$

should give the Hilbert series of the diagonal harmonic alternants and in particular it should evaluate to a polynomial in $q, t$ with positive integer coefficients. It is also shown in [11] that
a) $C_{n}(q, 1)=C_{n}(q) \quad$ and
b) $q^{\left(\begin{array}{c}n \\ 2\end{array}\right)} C_{n}(q, 1 / q)=\frac{1}{[n+1]_{q}}\left[\begin{array}{c}2 n \\ n\end{array}\right]_{q}$. 
For these reasons $C_{n}(q, t)$ has come to be referred to as the " $q, t$-Catalan".

Computer experimentations with $C_{n}(q, t)$ always yield a beautiful polynomial, symmetric in $q, t$. Moreover, the specialization in $4.3 \mathrm{a}$ ) suggests that there must be a pair of statistics on Dick paths, both having marginal distributions $q$-counting paths by area, with joint distribution given by $C_{n}(q, t)$. The construction of these statistics should make a challenging combinatorial problem. In fact, although the polynomiality of $C_{n}(q, t)$ can now be easily derived by combining 4.2 with Corollary 1.2 , even the mere positivity of $C_{n}(q, t)$ has remained an open problem to this date.

In this section we shall show that the identities in 1.12 lead to a relatively simple formula for $C_{n}(q, t)$ which does not involve $\nabla$ or the Macdonald polynomials. We include this result here in the hope that it may be helpful in further study of this remarkable polynomial.

To begin with we should note that a formula for $C_{n}(q, t)$ with the desired properties should come out of the identity in 2.6 by means of Theorem 1.1. In fact, expanding $e_{n}$ in terms of the basis in I.13 and then using 4.2 and 1.18 we should obtain an entirely new expression not only for $C_{n}(q, t)$ but for $\nabla e_{n}$ as well. Unfortunately, computer experimentation quickly reveals that the resulting expansion of $\nabla e_{n}$ is of forbidding complexity even for relatively small values of $n$. Nevertheless, a perusal of the tables of Schur function images by $\nabla$ revealed that certain coefficients of $\nabla e_{n}$ may be indirectly obtained from corresponding coefficients of $\nabla h_{n+1}$. In particular we can prove the following general identity:

$$
\left.\nabla e_{n}\right|_{S_{1^{n}}}=\left.\left(\frac{-1}{q t}\right)^{n} \nabla h_{n+1}\right|_{S_{1^{n+1}}} .
$$

This turns out to be a breakthrough since $h_{n+1}$ itself happens to have a relatively simple expansion in terms of the basis in I.13. More precisely we have

THEOREM 4.1. For $n \geq 0$

$$
h_{n+1}=\frac{1}{\tilde{M}^{n}} \sum_{i=0}^{n}\left(\begin{array}{c}
n \\
i
\end{array}\right)(-1)^{i} e_{1}^{n-i} D_{1}^{*} e_{1}^{i} \mathbf{1},
$$

and consequently

$$
\nabla h_{n+1}=\frac{1}{\tilde{M}^{n}} \sum_{i=0}^{n}\left(\begin{array}{c}
n \\
i
\end{array}\right)(-1)^{n-i} D_{1}^{n-i} e_{1} D_{1}^{i} \mathbf{1} .
$$

Proof. Note that I.12 $(i i)^{*}$, for $k=n$, can be written as

$$
D_{n+1}^{*}=\frac{1}{\tilde{M}}\left(e_{1} D_{n}^{*}-D_{n}^{*} e_{1}\right)
$$

A simple induction argument then yields that

$$
D_{n+1}^{*}=\frac{1}{\tilde{M}^{n}} \sum_{i=0}^{n}\left(\begin{array}{l}
n \\
i
\end{array}\right)(-1)^{i} e_{1}^{n-i} D_{1}^{*} e_{1}^{i},
$$

and 4.5 follows from 4.8 because I.10, for $k=n+1$ and $F=\mathbf{1}$, reduces to

$$
D_{n+1}^{*} \mathbf{1}=h_{n+1} \text {. }
$$

This given, 4.6 is obtained from 4.5 by repeated applications of $\mathrm{I} .12(\mathrm{iii})$ and $(\mathrm{iii})^{*}$. 
To prove 4.4 we need a few auxiliary identities.

Proposition 4.1.

$$
\begin{gathered}
e_{n}=\sum_{\mu \vdash n} \frac{\tilde{H}_{\mu}[X ; q, t] M B_{\mu}(q, t) \Pi_{\mu}(q, t)}{\tilde{h}_{\mu}(q, t) \tilde{h}_{\mu}^{\prime}(q, t)} \\
h_{n}=(-q t)^{n-1} \sum_{\mu \vdash n} \frac{\tilde{H}_{\mu}[X ; q, t] M B_{\mu}(1 / q, 1 / t) \Pi_{\mu}(q, t)}{\tilde{h}_{\mu}(q, t) \tilde{h}_{\mu}^{\prime}(q, t)}
\end{gathered}
$$

with $\Pi_{\mu}(q, t)$ as given by $I .3$.

Proof. Formula 4.9 was first derived in [11], but for sake of completeness we will sketch the proof here. We start with 1.2 a) and c) for $Y=1-u$ and get, by equating the homogeneous components of degree $n$ :

$$
\sum_{\rho \vdash n} \frac{p_{\rho}[X] p_{\rho}[1-u]}{(-1)^{|\rho|-l(\rho)} z_{\rho} p_{\rho}[M]}=\sum_{\mu \vdash n} \frac{\tilde{H}_{\mu}[X ; q, t] \prod_{s \in \mu}\left(1-u t^{l_{\mu}^{\prime}(s)} q^{a_{\mu}^{\prime}(s)}\right)}{\tilde{h}_{\mu}(q, t) \tilde{h}_{\mu}^{\prime}(q, t)}
$$

where we have used the specialization

$$
\tilde{H}_{\mu}[1-u ; q, t]=\prod_{s \in \mu}\left(1-u t^{l_{\mu}^{\prime}(s)} q^{a_{\mu}^{\prime}(s)}\right)
$$

a proof of which may be found in [12].

Now dividing both sides of 4.12 by $(1-u)$ and passing to the limit as $u \rightarrow 1$ we obtain

$$
\frac{p_{n}}{(-1)^{n-1} p_{n}[M]}=\sum_{\mu \vdash n} \frac{\tilde{H}_{\mu}[X ; q, t] \Pi_{\mu}(q, t)}{\tilde{h}_{\mu}(q, t) \tilde{h}_{\mu}^{\prime}(q, t)} .
$$

Next, from the definition I.10 of $D_{0}$, we get

$$
\left(1-D_{0}\right) p_{n}=(-1)^{n-1} p_{n}[M] e_{n} .
$$

At the same time I.12 (i) and I.4 give

$$
\left(1-D_{0}\right) \tilde{H}_{\mu}[X ; q, t]=M B_{\mu}(q, t) \tilde{H}_{\mu}[X ; q, t] .
$$

Thus 4.8 follows by applying $\left(1-D_{0}\right)$ to both sides of 4.13 .

To obtain 4.10 we start with the observation that $h_{n}=\omega e_{n}$ and, using 1.13 , derive from 4.8 that

$$
h_{n}=\sum_{\mu \vdash n} \frac{T_{\mu} \tilde{H}_{\mu}[X ; 1 / q, 1 / t] M B_{\mu}(q, t) \Pi_{\mu}(q, t)}{\tilde{h}_{\mu}(q, t) \tilde{h}_{\mu}^{\prime}(q, t)} .
$$

Making the replacements $t \rightarrow 1 / t$ and $q \rightarrow 1 / q$ and using the simple identities

$$
\tilde{h}_{\mu}(1 / q, 1 / t)=\frac{\tilde{h}_{\mu}(q, t)}{T_{\mu} t^{n}} \quad, \quad \tilde{h}_{\mu}^{\prime}(1 / q, 1 / t)=\frac{\tilde{h}_{\mu}^{\prime}(q, t)}{T_{\mu} q^{n}}
$$

formula 4.14 becomes

$$
h_{n}=\sum_{\mu \vdash n} \frac{(t q)^{n-1} T_{\mu} \tilde{H}_{\mu}[X ; q, t] M B_{\mu}(1 / q, 1 / t) \Pi_{\mu}(1 / q, 1 / t)}{\tilde{h}_{\mu}(q, t) \tilde{h}_{\mu}^{\prime}(q, t)},
$$


and since

$$
T_{\mu} \Pi_{\mu}(1 / q, 1 / t)=(-1)^{n-1} \Pi_{\mu}(q, t)
$$

we see that formula 4.15 is equivalent to 4.10 , and the proof is now complete.

This places us in a position to establish the following general identity.

TheOREM 4.2. For $n \geq 1$ we have

$$
\left(\nabla h_{n}\right)[1-u]=-u(-q t)^{n} \nabla e_{n-1}[1-u] .
$$

In particular we derive that for $1 \leq k \leq n$

$$
\left.\nabla h_{n}\right|_{S_{1^{k}, n-k}}=\left.(-q t)^{n-1}\left(\nabla e_{n-1}\right)\right|_{S_{1^{k-1}, n-k}} .
$$

Proof. Note first that 1.37 for $k=0$ gives

$$
B_{\mu}(1 / q, 1 / t)=\sum_{\nu \rightarrow \mu} c_{\mu, \nu}(1 / q, 1 / t) .
$$

Now from 1.38 we easily derive that

$$
c_{\mu, \nu}(1 / q, 1 / t)=c_{\mu, \nu}(q, t) \frac{T_{\nu}}{T_{\mu}} .
$$

Using this and 4.12 in 4.10 yields

$$
\begin{aligned}
& \left(\nabla h_{n}\right)[1-u] \\
= & (-q t)^{n-1} \sum_{\mu \vdash n} \frac{T_{\mu} \prod_{s \in \mu}\left(1-t^{l_{\mu}^{\prime}(s)} q^{a_{\mu}^{\prime}(s)}\right) M \Pi_{\mu}(q, t)}{\tilde{h}_{\mu}(q, t) \tilde{h}_{\mu}^{\prime}(q, t)} \sum_{\nu \rightarrow \mu} c_{\mu, \nu}(q, t) \frac{T_{\nu}}{T_{\mu}} \\
= & (-q t)^{n-1} \sum_{\nu \vdash n-1} \frac{T_{\nu} \prod_{s \in \nu}\left(1-t^{l_{\nu}^{\prime}(s)} q^{a_{\nu}^{\prime}(s)}\right) M \Pi_{\nu}(q, t)}{\tilde{h}_{\nu}(q, t) \tilde{h}_{\nu}^{\prime}(q, t)} \\
& \times \sum_{\mu \leftarrow \nu} c_{\mu, \nu}(q, t) \frac{\tilde{h}_{\nu}(q, t) \tilde{h}_{\mu}^{\prime}(q, t)}{\tilde{h}_{\nu}(q, t) \tilde{h}_{\mu}^{\prime}(q, t)}\left(1-u \frac{T_{\mu}}{T_{\nu}}\right)\left(1-\frac{T_{\mu}}{T_{\nu}}\right) \\
= & (-q t)^{n-1} \sum_{\nu \vdash n-1} \frac{T_{\nu} \prod_{s \in \nu}\left(1-t_{\nu}^{l_{\nu}^{\prime}(s)} q^{a_{\nu}^{\prime}(s)}\right) \Pi_{\nu}(q, t)}{\tilde{h}_{\nu}(q, t) \tilde{h}_{\nu}^{\prime}(q, t)} \sum_{\mu \leftarrow \nu} d_{\mu, \nu}(q, t)\left(1-u \frac{T_{\mu}}{T_{\nu}}\right)\left(1-\frac{T_{\mu}}{T_{\nu}}\right)
\end{aligned}
$$

where we have used 1.38 and 1.39 to get that

$$
M c_{\mu, \nu}(q, t) \frac{\tilde{h}_{\nu}(q, t) \tilde{h}_{\mu}^{\prime}(q, t)}{\tilde{h}_{\nu}(q, t) \tilde{h}_{\mu}^{\prime}(q, t)}=d_{\mu, \nu}(q, t) .
$$

Simple manipulations which use formula 1.40 for $k=0,1 \& 2$ finally give

$$
\sum_{\mu \leftarrow \nu} d_{\mu, \nu}(q, t)\left(1-u \frac{T_{\mu}}{T_{\nu}}\right)\left(1-\frac{T_{\mu}}{T_{\nu}}\right)=-u M B_{\nu}(q, t)
$$

Substituting this in 4.18 yields

$$
\left(\nabla h_{n}\right)[1-u]=-u(-q t)^{n-1} \sum_{\nu \vdash n-1} \frac{T_{\nu} \prod_{s \in \nu}\left(1-t^{l_{\nu}^{\prime}(s)} q^{a_{\nu}^{\prime}(s)}\right) M B_{\nu}(q, t) \Pi_{\nu}(q, t)}{\tilde{h}_{\nu}(q, t) \tilde{h}_{\nu}^{\prime}(q, t)},
$$


which is easily seen to imply 4.16 via $4.8,4.12$ and the definition of $\nabla$.

Now it is well known and easy to show that

$$
S_{\lambda}[1-u]= \begin{cases}(-u)^{k}(1-u) & \text { if } \lambda=\left(n-k, 1^{k}\right) \\ 0 & \text { otherwise. }\end{cases}
$$

This given, formula 4.17 immediately follows by dividing both sides of 4.16 by $(1-u)$ and equating coefficients of $u^{k}$.

As corollary of 4.17 we obtain our desired formula for the $q$, $t$-Catalan.

THEOREM 4.3.

$$
C_{n}(q, t)=\left.\frac{1}{M^{n}} \sum_{i=0}^{n}\left(\begin{array}{c}
n \\
i
\end{array}\right)(-1)^{i} D_{1}^{n-i} e_{1} D_{1}^{i} \mathbf{1}\right|_{S_{1^{n+1}}}
$$

Proof. We can clearly see from 4.1 and 4.8 that $D H_{n}=\nabla e_{n}$. Thus from 4.2 we get that

$$
C_{n}(q, t)=\left.\nabla e_{n}\right|_{S_{1 n}} .
$$

On the other hand 4.17 with $n \rightarrow n+1$ and $k=0$ specializes to 4.4 giving

$$
\begin{aligned}
C_{n}(q, t) & =\left.\left(\frac{-1}{q t}\right)^{n} \nabla h_{n+1}\right|_{S_{1^{n+1}}} \\
& =\left.\left(\frac{-1}{q t}\right)^{n} \frac{1}{\tilde{M}^{n}} \sum_{i=0}^{n}\left(\begin{array}{c}
n \\
i
\end{array}\right)(-1)^{n-i} D_{1}^{n-i} e_{1} D_{1}^{i} \mathbf{1}\right|_{S_{1^{n+1}}}
\end{aligned}
$$

where the last equality is due to 4.6 . This proves 4.19 since $q t \tilde{M}=M$.

It is worth noting that the same idea can be used to obtain a recursive way of computing the $q, t$-Catalan. More precisely we have

THEOREM 4.4. Let $\Gamma_{n}$ be a sequence of operators defined by the following conditions:

$$
\begin{aligned}
& \text { a) } \Gamma_{1}=D_{2} \\
& \text { b) } \Gamma_{n}=\frac{1}{M}\left(D_{1} \Gamma_{n-1}-\Gamma_{n-1} D_{1}\right) .
\end{aligned}
$$

Then

$$
C_{n}(q, t)=\left.\Gamma_{n} \mathbf{1}\right|_{S_{1^{n+1}}}
$$

Proof. Conjugating 4.7 by $\nabla$ we get (using I.12 $(i i i)^{*}$ and (iii))

$$
\nabla D_{n+1}^{*} \nabla^{-1}=\frac{1}{\tilde{M}}\left(\left(-D_{1}\right) \nabla D_{n}^{*} \nabla^{-1}-\nabla D_{n}^{*} \nabla^{-1}\left(-D_{1}\right)\right)
$$

This given, note that if we set

$$
\Gamma_{n}=\left(\frac{-1}{q t}\right)^{n} \nabla D_{n+1}^{*} \nabla^{-1}
$$


then $\left(\right.$ since $\left.\nabla^{-1} \mathbf{1}=\mathbf{1}\right)$ we get

$$
\Gamma_{n} \mathbf{1}=\left(\frac{-1}{q t}\right)^{n} \nabla D_{n+1}^{*} \mathbf{1}=\left(\frac{-1}{q t}\right)^{n} \nabla h_{n+1}
$$

and this gives 4.21 .

On the other hand 4.23 converts 4.22 into

$$
\Gamma_{n}=\left(\frac{-1}{q t}\right) \frac{1}{\tilde{M}}\left(\left(-D_{1}\right) \Gamma_{n-1}-\Gamma_{n-1}\left(-D_{1}\right)\right)
$$

which is simply another way of writing $4.20 \mathrm{~b}$ ). Finally note that 4.23 gives

$$
\Gamma_{0}=\nabla D_{1}^{*} \nabla^{-1}=e_{1}
$$

and from $4.21 \mathrm{~b}$ ) we get

$$
\Gamma_{1}=\frac{1}{M}\left(D_{1} e_{1}-e_{1} D_{1}\right)
$$

Thus 4.20 a) follows from I.12 (ii).

5. Plethystic form for Macdonald operators of higher index. The main goal of this section is to show that the operators $\Delta_{F}$ studied in section 1 , and in particular $\nabla$ itself, may also be given a plethystic form. We believe that the arguments of the proof may be as interesting as the result itself, since the latter, because of its inherent complexity, may be only of theoretical significance.

To begin we need to review notation and introduce some auxiliary variables. Recall that it is customary to set (see [16] p. xiv)

$$
(a ; t)_{n}=\prod_{i=1}^{n}\left(1-a t^{i-1}\right) \quad(t ; t)_{n}=\prod_{i=1}^{n}\left(1-t^{i}\right) .
$$

This given, for an alphabet $Z_{r}=z_{1}+\cdots+z_{r}$ set

$$
\Delta_{r}^{\prime}\left(Z_{r} ; q, t\right)=\prod_{j \neq k}\left(z_{j} z_{k}^{-1} ; q\right)_{\infty} / \prod_{j \neq k}\left(t z_{j} z_{k}^{-1} ; q\right)_{\infty}=\Omega\left[-\frac{1-t}{1-q} \sum_{j \neq k} \frac{z_{j}}{z_{k}}\right]
$$

where here and after, $j \neq k$ runs over $1 \leq j, k \leq r$.

We shall also make crucial use here of the constant term scalar product introduced in [22, Chapter VI.9] by setting, for any two polynomials $f$ and $g$,

$$
\langle f, g\rangle_{r, q, t}^{\prime}=\left.\frac{1}{r !} f\left(z_{1}, \ldots, z_{r}\right) g\left(z_{1}^{-1}, \ldots, z_{r}^{-1}\right) \Delta_{r}^{\prime}\left(Z_{r} ; q, t\right)\right|_{z^{0}}
$$

where the symbol " $\left.\right|_{z^{0}}$ " represents the operation of taking the constant term in the preceding expression.

For any composition $\lambda$, define the alphabets

$$
Z_{\lambda}=\sum_{i=1}^{l(\lambda)} \sum_{j=1}^{\lambda_{i}} z_{i j}, \quad Z_{\lambda}^{-1}=\sum_{i=1}^{l(\lambda)} \sum_{j=1}^{\lambda_{i}} z_{i j}^{-1} .
$$


Also let $\lambda !=\prod_{i} \lambda_{i} !, n\left(\lambda^{\prime}\right)=\sum_{i}\left(\begin{array}{c}\lambda_{i} \\ 2\end{array}\right)$ and set

$$
E_{\mu}(q, t)=\sum_{i \geq 1} q^{\mu_{i}} t^{i-1}=\frac{-D_{\mu}(q, t)}{1-t}=(q-1) B_{\mu}(q, t)+\frac{1}{1-t} .
$$

Our main result may be stated as follows.

TheOREM 5.1. Let $\lambda$ be a composition and $P[X]$ a symmetric function. Define the linear operator $\Delta_{\lambda}$ by setting

$$
\begin{aligned}
\Delta_{\lambda} P[X]=\frac{t^{n\left(\lambda^{\prime}\right)}}{\lambda !(1-t)^{|\lambda|}} P\left[X+(q-1)(t-1) Z_{\lambda}^{-1}\right] \Omega\left[-X Z_{\lambda}\right] \\
\quad \times \Omega\left[-(t-1)(q-1) \sum_{i<j}\left(z_{i 1}^{-1}+\cdots+z_{i, \lambda_{i}}^{-1}\right)\left(z_{j 1}+\cdots+z_{j, \lambda_{j}}\right)\right] \\
\quad \times\left.\Omega\left[(t-1) \sum_{i=1}^{l(\lambda)} \sum_{\substack{1 \leq j, k \leq \lambda_{i} \\
j \neq k}} \frac{z_{i j}}{z_{i k}}\right]\right|_{z^{0}} .
\end{aligned}
$$

Then for all partitions $\mu$ and $\lambda$ we have

$$
\Delta_{\lambda} \tilde{H}_{\mu}[X ; q, t]=e_{\lambda}\left[E_{\mu}(q, t)\right] \tilde{H}_{\mu}[X ; q, t] .
$$

The proof will be obtained by combining some of the basic identities satisfied by Macdonald operators together with a number of auxiliary propositions we are about to establish.

Let us recall that in [22, Chapter VI.3], Macdonald defines an operator $D_{n}^{r}$ that we will call $D_{r}^{(n)}$. Using plethystic notation, it may be written as

$$
D_{r}^{(n)}(q, t) P\left[X_{n}\right]=\sum_{|I|=r} A_{I}\left(X_{n} ; t\right) P\left[X_{n}+(q-1) X_{I}\right]
$$

where $X_{n}$ is an $n$ letter alphabet and for any $r$-subset $I$ of $\{1, \ldots, n\}$, we set $X_{I}=$ $\sum_{i \in I} x_{i}$ and

$$
A_{I}\left(X_{n} ; t\right)=\prod_{i \in I ; j \notin I} \frac{t x_{i}-x_{j}}{x_{i}-x_{j}}
$$

We will present another form of these operators that does not require us to take subalphabets of $X_{n}$.

A generating function for these operators is

$$
D^{(n)}(u ; q, t)=\sum_{r=0}^{n} u^{r} D_{r}^{(n)}(q, t) .
$$

Macdonald shows [22, p. 324] that for $l(\mu) \leq n$, we have

$$
D^{(n)}(u ; q, t) P_{\mu}(x ; q, t)=\left(\prod_{i=1}^{n}\left(1+u q^{\mu_{i}} t^{n-i}\right)\right) P_{\mu}(x ; q, t) .
$$


By taking the coefficient of $u^{r}$ on both sides, we obtain

$$
D_{r}^{(n)}(q, t) P_{\mu}(x ; q, t)=e_{r}\left[\sum_{i=1}^{n} q^{\mu_{i}} t^{n-i}\right] P_{\mu}(x ; q, t) .
$$

These operators depend on $n$, the size of the alphabet. We will "stabilize" them with respect to $n$ by reexpressing them in terms of $E_{\mu}(q, t)$. To this end let

$$
E_{\mu}^{\prime}(q, t)=E_{\mu}\left(q, t^{-1}\right)
$$

Note that we have

$$
\begin{aligned}
e_{r}\left[E_{\mu}^{\prime}(q, t)\right]=e_{r}\left[\sum_{i=1}^{\infty} q^{\mu_{i}} t^{1-i}\right] & =t^{-(n-1) r} e_{r}\left[\sum_{i=1}^{n} q^{\mu_{i}} t^{n-i}+\sum_{i=n+1}^{\infty} t^{n-i}\right] \\
& =t^{-(n-1) r} \sum_{k=0}^{r} e_{r-k}\left[\sum_{i=1}^{\infty} t^{-i}\right] e_{k}\left[\sum_{i=1}^{n} q^{\mu_{i}} t^{n-i}\right] \\
& =t^{-(n-1) r} \sum_{k=0}^{r} \frac{(-1)^{r-k}}{(t ; t)_{r-k}} e_{k}\left[\sum_{i=1}^{n} q^{\mu_{i}} t^{n-i}\right] .
\end{aligned}
$$

We may therefore stabilize the Macdonald operators by setting

$$
\widehat{D}_{r}^{(n)}=t^{-(n-1) r} \sum_{k=0}^{r} \frac{(-1)^{r-k}}{(t ; t)_{r-k}} D_{k}^{(n)} .
$$

This will produce eigenvalues that only depend on $\mu$ and $r$, but not $n$. More precisely we have

$$
\widehat{D}_{r}^{(n)} P_{\mu}\left[X_{n} ; q, t\right]=e_{r}\left[E_{\mu}^{\prime}(q, t)\right] P_{\mu}\left[X_{n} ; q, t\right] .
$$

For example, Macdonald's one stable operator $E[22$, p. 321] may be expressed $E=$ $\widehat{D}_{1}+\frac{1}{1-t}$. To recover the $D_{r}^{(n)}$ 's from the $\widehat{D}_{r}^{(n)}$ 's, we need the following well-known and easily derived variation of Möbius inversion:

Lemma 5.1. Let $f_{r}, g_{r}$ be sequences, $r=0,1, \ldots, n$. Then

$$
g_{r}=\sum_{k=0}^{r} \frac{f_{k}}{(t ; t)_{r-k}} \quad \text { for } r=0, \ldots, n
$$

if and only if

$$
f_{r}=\sum_{k=0}^{r} \frac{(-1)^{k} t^{\left(\begin{array}{c}
k \\
2
\end{array}\right)} g_{r-k}}{(t ; t)_{k}}=\sum_{k=0}^{r} \frac{(-1)^{r-k} t^{\left(\begin{array}{r}
r-k \\
2
\end{array}\right)} g_{k}}{(t ; t)_{r-k}} \quad \text { for } r=0, \ldots, n .
$$

It follows that the original Macdonald operators may be recovered from the stabilized ones via

$$
D_{r}^{(n)}=\sum_{k=0}^{r} \frac{t^{\left(\begin{array}{r}
r-k \\
2
\end{array}\right)+k(n-1)}}{(t ; t)_{r-k}} \widehat{D}_{k}^{(n)}
$$


For any symmetric function $f$, we are now in a position to construct operators $\widehat{D}_{f}$ such that for any partition $\mu$,

$$
\widehat{D}_{f} P_{\mu}[X ; q, t]=f\left[E_{\mu}^{\prime}(q, t)\right] P_{\mu}[X ; q, t]
$$

Indeed, when $f=e_{\lambda_{1}} \cdots e_{\lambda_{k}}$ and the alphabet is of the form $X=X_{n}$, we may take

$$
\widehat{D}_{\lambda}^{(n)}=\widehat{D}_{e_{\lambda}}^{(n)}=\widehat{D}_{\lambda_{1}}^{(n)} \cdots \widehat{D}_{\lambda_{k}}^{(n)}
$$

Since the elementary symmetric functions are a basis of all symmetric functions, we may form $\widehat{D}_{f}^{(n)}$ as a linear combination of these.

We shall find new plethystic forms of the operators $\widehat{D}_{\lambda}^{(n)}$ that do not depend on $n$.

Proposition 5.1. Let $\lambda$ be a composition and $P[X]$ a symmetric function. Set

$$
\begin{aligned}
& \widehat{D}_{\lambda} P[X]=\frac{1}{\lambda !\left(1-t^{-1}\right)^{|\lambda|} t^{n\left(\lambda^{\prime}\right)}} P\left[X+(q-1) Z_{\lambda}^{-1}\right] \Omega\left[\left(1-t^{-1}\right) X Z_{\lambda}\right] \\
& \times \Omega\left[\left(1-t^{-1}\right)(q-1) \sum_{i<j}\left(z_{i 1}^{-1}+\cdots+z_{i, \lambda_{i}}^{-1}\right)\left(z_{j 1}+\cdots+z_{j, \lambda_{j}}\right)\right] \\
& \times\left.\Omega\left[\left(t^{-1}-1\right) \sum_{i=1}^{l(\lambda)} \sum_{\substack{1 \leq j, k \leq \lambda_{i} \\
j \neq k}} \frac{z_{i j}}{z_{i k}}\right]\right|_{z^{0}} .
\end{aligned}
$$

Then for all $n \geq|\lambda|$,

$$
\widehat{D}_{\lambda} P\left[X_{n}\right]=\widehat{D}_{\lambda}^{(n)} P\left[X_{n}\right]
$$

and in particular, for all partitions $\mu$ and $\lambda$,

$$
\widehat{D}_{\lambda} P_{\mu}[X ; q, t]=e_{\lambda}\left[E_{\mu}^{\prime}(q, t)\right] P_{\mu}[X ; q, t] .
$$

The operators $\widehat{D}_{f}$ satisfying 5.15 are then $\widehat{D}_{f}=\sum_{\lambda} a_{\lambda} \widehat{D}_{\lambda}$, where the coefficients $a_{\lambda}$ are given by $f=\sum_{\lambda} a_{\lambda} e_{\lambda}$.

Proof. We first do this for the case $\lambda=(r)$. The general case will follow by composing the resulting operators. The definition 5.16 in terms of the alphabet $Z_{r}$ is (5.19)

$$
\widehat{D}_{r} P[X]=\left.\frac{1}{r !\left(1-t^{-1}\right)^{r} t^{\left(\begin{array}{r}
r \\
2
\end{array}\right)}} P\left[X+(q-1) Z_{r}^{-1}\right] \Omega\left[\left(1-t^{-1}\right) X Z_{r}\right] \Omega\left[\left(t^{-1}-1\right) \sum_{\substack{1 \leq j, k \leq r \\
j \neq k}} \frac{z_{j}}{z_{k}}\right]\right|_{z^{0}},
$$

and we are to show that $\widehat{D}_{r} P\left[X_{n}\right]=\widehat{D}_{r}^{(n)} P\left[X_{n}\right]$ for all $n \geq r$.

Consider the modified Macdonald kernel

$$
\Omega\left[\frac{1-t^{-1}}{q-1} X_{n} Y\right]=\sum_{\mu: l(\mu) \leq n} P_{\mu}\left[X_{n} ; q, t^{-1}\right] Q_{\mu}\left[-Y ; q, t^{-1}\right]
$$


Apply $\widehat{D}_{r}^{(n)}$ to it:

$$
\begin{aligned}
& \widehat{D}_{r}^{(n)} \Omega\left[\frac{1-t^{-1}}{q-1} X_{n} Y\right] \\
= & t^{-(n-1) r} \sum_{k=0}^{r} \frac{(-1)^{r-k}}{(t ; t)_{r-k}} D_{k}^{(n)} \Omega\left[\frac{1-t^{-1}}{q-1} X_{n} Y\right] \\
= & t^{-(n-1) r} \sum_{k=0}^{r} \frac{(-1)^{r-k}}{(t ; t)_{r-k}} \sum_{|I|=k} A_{I}\left(X_{n} ; t\right) \Omega\left[\frac{1-t^{-1}}{q-1}\left(X_{n}+(q-1) X_{I}\right) Y\right] \\
= & t^{-(n-1) r} \Omega\left[\frac{1-t^{-1}}{q-1} X_{n} Y\right] \sum_{k=0}^{r} \frac{(-1)^{r-k}}{(t ; t)_{r-k}} \sum_{|I|=k} A_{I}\left(X_{n} ; t\right) \Omega\left[\left(1-t^{-1}\right) X_{I} Y\right] .
\end{aligned}
$$

To evaluate the inside summation

$$
\sum_{|I|=k} A_{I}\left(X_{n} ; t\right) \Omega\left[\left(1-t^{-1}\right) X_{I} Y\right]=\sum_{|I|=k} A_{I}\left(X_{n} ; t\right) \sum_{\mu} P_{\mu}\left(X_{I} ; 0, t^{-1}\right) Q_{\mu}\left(Y ; 0, t^{-1}\right),
$$

we apply Proposition 3.1 of [15]: for $k \leq n$ and all $\mu$,

$$
\sum_{|I|=k} A_{I}(x ; t) P_{\mu}\left[X_{I} ; 0, t^{-1}\right]=t^{\left(\begin{array}{c}
k \\
2
\end{array}\right)+(n-k) l(\mu)}\left[\begin{array}{l}
n-l(\mu) \\
k-l(\mu)
\end{array}\right]_{t} P_{\mu}\left[X_{n} ; 0, t^{-1}\right] .
$$

(The right side is 0 when $l(\mu) \leq k \leq n$ fails to hold.) This gives that 5.21 equals

$$
\begin{aligned}
& \sum_{\mu} t^{\left(\begin{array}{c}
k \\
2
\end{array}\right)+(n-k) l(\mu)}\left[\begin{array}{l}
n-l(\mu) \\
k-l(\mu)
\end{array}\right]_{t} P_{\mu}\left[X_{n} ; 0, t^{-1}\right] Q_{\mu}\left[Y ; 0, t^{-1}\right] \\
& =\sum_{m=0}^{n} t_{\left(\begin{array}{c}
k \\
2
\end{array}\right)+(n-k) m}\left[\begin{array}{l}
n-m \\
k-m
\end{array}\right]_{t} \sum_{\mu: l(\mu)=m} P_{\mu}\left[X_{n} ; 0, t^{-1}\right] Q_{\mu}\left[Y ; 0, t^{-1}\right] .
\end{aligned}
$$

The $m$ summation may be restricted to $0 \leq m \leq k$ because the $t$-binomial coefficient vanishes otherwise. Plugging 5.22 back into 5.20 and then reversing the order of the $k, m$ summations gives

$$
\begin{aligned}
& \frac{\widehat{D}_{r}^{(n)} \Omega\left[\frac{1-t^{-1}}{q-1} X_{n} Y\right]}{\Omega\left[\frac{1-t^{-1}}{q-1} X_{n} Y\right]} \\
& =t^{-(n-1) r} \sum_{k=0}^{r} \frac{(-1)^{r-k}}{(t ; t)_{r-k}} \sum_{m=0}^{k} t^{\left(\begin{array}{c}
k \\
2
\end{array}\right)+(n-k) m}\left[\begin{array}{l}
n-m \\
k-m
\end{array}\right]_{t} \sum_{\mu: l(\mu)=m} P_{\mu}\left[X_{n} ; 0, t^{-1}\right] Q_{\mu}\left[Y ; 0, t^{-1}\right] \\
& =t^{-(n-1) r} \sum_{m=0}^{r}(-1)^{r-m} t^{\left(\begin{array}{c}
m \\
2
\end{array}\right)+m(n-m)}\left(\sum_{k=m}^{r} \frac{(-1)^{k-m}}{(t ; t)_{r-k}} t^{\left(\begin{array}{c}
k-m \\
2
\end{array}\right)}\left[\begin{array}{l}
n-m \\
k-m
\end{array}\right]_{t}\right) \\
& \times\left(\sum_{\mu: l(\mu)=m} P_{\mu}\left[X_{n} ; 0, t^{-1}\right] Q_{\mu}\left[Y ; 0, t^{-1}\right]\right) \text {. }
\end{aligned}
$$

The details of evaluating the parenthesized sums in 5.23 will follow this proof. The 
$k$-sum may be simplified by 5.42 to

$$
\sum_{k=m}^{r} \frac{(-1)^{k-m}}{(t ; t)_{r-k}} t^{\left(\begin{array}{c}
k-m \\
2
\end{array}\right)}\left[\begin{array}{l}
n-m \\
k-m
\end{array}\right]_{t}=\sum_{k=0}^{r-m} \frac{(-1)^{k}}{(t ; t)_{r-m-k}} t^{\left(\begin{array}{c}
k \\
2
\end{array}\right)}\left[\begin{array}{c}
n-m \\
k
\end{array}\right]_{t}=\frac{t^{(r-m)(n-m)}}{(t ; t)_{r-m}}
$$

so that the right side of 5.23 equals

$$
t^{r} \sum_{m=0}^{r} \frac{(-1)^{r-m} t^{\left(\begin{array}{c}
m \\
2
\end{array}\right)-m r}}{(t ; t)_{r-m}} \sum_{\mu: l(\mu)=m} P_{\mu}\left[X_{n} ; 0, t^{-1}\right] Q_{\mu}\left[Y ; 0, t^{-1}\right]
$$

We will evaluate this sum in 5.34. It is a long computation involving the scalar product 5.2 , and it equals

$$
\left.\frac{1}{r !\left(1-t^{-1}\right)^{r} t^{\left(\begin{array}{r}
2 \\
2
\end{array}\right)}} \Omega\left[\left(1-t^{-1}\right) X_{n} Z_{r}\right] \Omega\left[\left(1-t^{-1}\right) Y Z_{r}^{-1}\right] \Omega\left[\left(t^{-1}-1\right) \sum_{j \neq k} \frac{z_{j}}{z_{k}}\right]\right|_{z^{0}} .
$$

Equating the left side of 5.23 with 5.24 and rearranging gives

$$
\begin{aligned}
& \widehat{D}_{r}^{(n)} \Omega\left[\frac{1-t^{-1}}{q-1} X_{n} Y\right]=\frac{1}{r !\left(1-t^{-1}\right)^{r} t^{(r} \begin{array}{l}
2 \\
2
\end{array}} \Omega\left[\frac{1-t^{-1}}{q-1}\left(X_{n}+(q-1) Z_{r}^{-1}\right) Y\right] \\
& \times\left.\Omega\left[\left(1-t^{-1}\right) X_{n} Z_{r}\right] \Omega\left[\left(t^{-1}-1\right) \sum_{j \neq k} \frac{z_{j}}{z_{k}}\right]\right|_{z^{0}} \\
& =\widehat{D}_{r} \Omega\left[\frac{1-t^{-1}}{q-1} X_{n} Y\right] \text {. }
\end{aligned}
$$

From this we conclude that $\widehat{D}_{r}^{(n)} P\left[X_{n}\right]=\widehat{D}_{r} P\left[X_{n}\right]$ for any symmetric function $P[X]$, so 5.17 holds for $\lambda=(r)$.

Now for an arbitrary composition $\lambda=\left(\lambda_{1}, \ldots, \lambda_{l}\right)$, we must check that $\widehat{D}_{\lambda}=$ $\widehat{D}_{\lambda_{1}} \cdots \widehat{D}_{\lambda_{l}}$. We induct on $l$. Set $\rho=\left(\lambda_{1}, \ldots, \lambda_{l-1}\right), r=\lambda_{l}$, and verify that $\widehat{D}_{\rho} \widehat{D}_{r} P[X]=\widehat{D}_{\lambda} P[X]$. We break up the alphabet $Z_{\lambda}=U_{\rho}+V_{r}$ where $U_{\rho}=$ $\sum_{i=1}^{l(\rho)} \sum_{j=1}^{\lambda_{i}} z_{i j}$ and $V_{r}=\sum_{j=1}^{r} z_{\lambda_{l}, j}$. Then by 5.19 ,

$$
\widehat{D}_{r} P[X]=\left.\frac{1}{r !\left(1-t^{-1}\right)^{r} t^{\left(\begin{array}{c}
r \\
2
\end{array}\right)}} P\left[X+(q-1) V_{r}^{-1}\right] \Omega\left[\left(1-t^{-1}\right) X V_{r}\right] \Omega\left[\left(t^{-1}-1\right) \sum_{\substack{1 \leq j, k \leq r \\
j \neq k}} \frac{z_{l j}}{z_{l k}}\right]\right|_{v^{0}}
$$


so by 5.16 ,

$$
\begin{aligned}
& \widehat{D}_{\rho} \widehat{D}_{r} P[X] \\
& =\frac{P\left[X+(q-1)\left(U_{\rho}^{-1}+V_{r}^{-1}\right)\right]}{r ! \rho !\left(1-t^{-1}\right)^{|\rho|+r} t^{n\left(\rho^{\prime}\right)+\left(\begin{array}{c}
r \\
2
\end{array}\right)}} \Omega\left[\left(1-t^{-1}\right)\left(X+(q-1) U_{\rho}^{-1}\right) V_{r}\right] \\
& \times \Omega\left[\left(t^{-1}-1\right) \sum_{1 \leq j, k \leq r} \frac{z_{l j}}{z_{l k}}\right] \Omega\left[\left(1-t^{-1}\right) X U_{\rho}\right] \\
& j \neq k \\
& \times \Omega\left[\left(1-t^{-1}\right)(q-1) \sum_{1 \leq i<j \leq l(\rho)}\left(z_{i 1}^{-1}+\cdots+z_{i, \rho_{i}}^{-1}\right)\left(z_{j 1}+\cdots+z_{j, \rho_{j}}\right)\right] \\
& \times\left.\Omega\left[\left(t^{-1}-1\right) \sum_{i=1}^{l(\rho)} \sum_{1 \leq j, k \leq \rho_{i}} \frac{z_{i j}}{z_{i k}}\right]\right|_{u^{0} v^{0}} \\
& j \neq k \\
& =\frac{P\left[X+(q-1)\left(U_{\rho}^{-1}+V_{r}^{-1}\right)\right]}{\lambda !\left(1-t^{-1}\right)^{|\lambda|} t^{n\left(\lambda^{\prime}\right)}} \Omega\left[\left(1-t^{-1}\right) X\left(U_{\rho}+V_{r}\right)\right] \\
& \times \Omega\left[\left(1-t^{-1}\right)(q-1)\left(U_{\rho}^{-1} V_{r}+\sum_{1 \leq i<j \leq l(\rho)}\left(z_{i 1}^{-1}+\cdots+z_{i, \rho_{i}}^{-1}\right)\left(z_{j 1}+\cdots+z_{j, \rho_{j}}\right)\right)\right] \\
& \times\left.\Omega\left[\left(t^{-1}-1\right)\left(\sum_{i=1}^{l(\rho)} \sum_{\substack{1 \leq j, k \leq \rho_{i} \\
j \neq k}} \frac{z_{i j}}{z_{i k}}+\sum_{\substack{1 \leq j, k \leq r \\
j \neq k}} \frac{z_{l j}}{z_{l k}}\right)\right]\right|_{u^{0} v^{0}}
\end{aligned}
$$

and on combining the alphabets into $Z_{\lambda}=U_{\rho}+V_{r}$, it simplifies to 5.16 as required.

Proof of Theorem 5.1. Since Macdonald's $J_{\mu}(x ; q, t)$ is a scalar multiple of $P_{\mu}(x ; q, t)$, it follows from 5.16 and 5.18 that

$$
\begin{aligned}
\widehat{D}_{\lambda} J_{\mu}[X ; q, t]=\frac{1}{\lambda !\left(1-t^{-1}\right)^{|\lambda|} t^{n\left(\lambda^{\prime}\right)}} J_{\mu}\left[X+(q-1) Z_{\lambda}^{-1} ; q, t\right] \Omega\left[\left(1-t^{-1}\right) X Z_{\lambda}\right] \\
\quad \times \Omega\left[\left(1-t^{-1}\right)(q-1) \sum_{i<j}\left(z_{i 1}^{-1}+\cdots+z_{i, \lambda_{i}}^{-1}\right)\left(z_{j 1}+\cdots+z_{j, \lambda_{j}}\right)\right] \\
\quad \times\left.\Omega\left[\left(t^{-1}-1\right) \sum_{i=1}^{l(\lambda)} \sum_{\substack{1 \leq j, k \leq \lambda_{i} \\
j \neq k}} \frac{z_{i j}}{z_{i k}}\right]\right|_{z^{0}} \\
=e_{\lambda}\left[E_{\mu}^{\prime}(q, t)\right] J_{\mu}[X ; q, t] .
\end{aligned}
$$


We will transform this from $J_{\mu}$ to $\tilde{H}_{\mu}$ using I.6. Replace $X$ by $X /(1-t)$ in 5.27 : (5.28)

$$
\begin{gathered}
\frac{1}{\lambda !\left(1-t^{-1}\right)^{|\lambda|} t^{n\left(\lambda^{\prime}\right)}} J_{\mu}\left[\frac{X+(q-1)(1-t) Z_{\lambda}^{-1}}{1-t} ; q, t\right] \Omega\left[-t X Z_{\lambda}\right] \\
\times \Omega\left[\left(1-t^{-1}\right)(q-1) \sum_{i<j}\left(z_{i 1}^{-1}+\cdots+z_{i, \lambda_{i}}^{-1}\right)\left(z_{j 1}+\cdots+z_{j, \lambda_{j}}\right)\right] \\
\times\left.\Omega\left[\left(t^{-1}-1\right) \sum_{i=1}^{l(\lambda)} \sum_{\substack{1 \leq j, k \leq \lambda_{i} \\
j \neq k}} \frac{z_{i j}}{z_{i k}}\right]\right|_{z^{0}} \\
=e_{\lambda}\left[E_{\mu}^{\prime}(q, t)\right] J_{\mu}\left[\frac{X}{1-t} ; q, t\right] .
\end{gathered}
$$

Replace $t$ by $t^{-1}$, multiply both sides by $t^{n(\mu)}$, and express it in terms of $\tilde{H}_{\mu}$ via I.6: (5.29)

$$
\begin{aligned}
\frac{t^{n\left(\lambda^{\prime}\right)}}{\lambda !(1-t)^{|\lambda|}} \tilde{H}_{\mu}\left[X+(q-1)\left(1-t^{-1}\right) Z_{\lambda}^{-1} ; q, t\right] \Omega\left[-t^{-1} X Z_{\lambda}\right] \\
\times \Omega\left[-(t-1)(q-1) \sum_{i<j}\left(z_{i 1}^{-1}+\cdots+z_{i, \lambda_{i}}^{-1}\right)\left(z_{j 1}+\cdots+z_{j, \lambda_{j}}\right)\right] \\
\times\left.\Omega\left[(t-1) \sum_{i=1}^{l(\lambda)} \sum_{\substack{1 \leq j, k \leq \lambda_{i} \\
j \neq k}} \frac{z_{i j}}{z_{i k}}\right]\right|_{z^{0}} \\
=e_{\lambda}\left[E_{\mu}(q, t)\right] \tilde{H}_{\mu}[X ; q, t] .
\end{aligned}
$$

Replace $Z_{\lambda}$ by $t Z_{\lambda}$. The constant term with respect to $Z_{\lambda}$ is unaffected, and 5.29 is transformed to

$$
\begin{aligned}
\frac{t^{n\left(\lambda^{\prime}\right)}}{\lambda !(1-t)^{|\lambda|}} \tilde{H}_{\mu}[ & \left.+(q-1)(t-1) Z_{\lambda}^{-1} ; q, t\right] \Omega\left[-X Z_{\lambda}\right] \\
& \times \Omega\left[-(t-1)(\dot{q}-1) \sum_{i<j}\left(z_{i 1}^{-1}+\cdots+z_{i, \lambda_{i}}^{-1}\right)\left(z_{j 1}+\cdots+z_{j, \lambda_{j}}\right)\right] \\
& \times\left.\Omega\left[(t-1) \sum_{i=1}^{l(\lambda)} \sum_{\substack{1 \leq j, k \leq \lambda_{i} \\
j \neq k}} \frac{z_{i j}}{z_{i k}}\right]\right|_{z^{0}} \\
& =e_{\lambda}\left[E_{\mu}(q, t)\right] \tilde{H}_{\mu}[X ; q, t]
\end{aligned}
$$

so that 5.6 holds.

Proposition 5.2. Define a linear operator

$$
\nabla_{n}=\sum_{|\lambda| \leq n} e_{n-|\lambda|}\left[\frac{1}{(1-t)(1-q)}\right] m_{\lambda}\left[\frac{1}{q-1}\right] \Delta_{\lambda} .
$$

Then if $P[X]$ is a symmetric polynomial that is homogeneous of degree $n$,

$$
\nabla_{n} P[X]=\nabla P[X] .
$$


Proof. Since

$$
T_{\mu}=e_{n}\left[B_{\mu}\right]=e_{n}\left[\frac{E_{\mu}-\frac{1}{1-t}}{q-1}\right],
$$

an operator equivalent to $\nabla$ on homogeneous polynomials of degree $n$ is $\Delta_{f}$, where

$$
f=\sum_{|\lambda| \leq n} a_{\lambda}(q, t) e_{\lambda}
$$

where the coefficients $a_{\lambda}(q, t)$ are defined by

$$
e_{n}\left[\frac{X-\frac{1}{1-t}}{q-1}\right]=\sum_{\lambda} a_{\lambda}(q, t) e_{\lambda}[X] .
$$

We expand this as follows.

$$
\begin{aligned}
e_{n}\left[\frac{X-\frac{1}{1-t}}{q-1}\right] & =\sum_{r=0}^{n} e_{r}\left[\frac{X}{q-1}\right] e_{n-r}\left[\frac{1}{(1-t)(1-q)}\right] \\
& =\sum_{r=0}^{n} e_{n-r}\left[\frac{1}{(1-t)(1-q)}\right] \sum_{\lambda \vdash r} m_{\lambda}\left[\frac{1}{q-1}\right] e_{\lambda}[X] \\
& =\sum_{|\lambda| \leq n}\left(e_{n-|\lambda|}\left[\frac{1}{(1-t)(1-q)}\right] m_{\lambda}\left[\frac{1}{q-1}\right]\right) e_{\lambda}[X]
\end{aligned}
$$

We now evaluate the sums used in the proof of Proposition 5.1.

Proposition 5.3. For $r \geq 0$, and any alphabets $X, Y$,

$$
\begin{aligned}
& \sum_{m=0}^{r} \frac{1}{(t ; t)_{r-m}} \cdot \sum_{\mu: l(\mu)=m} P_{\mu}[X ; 0, t] Q_{\mu}[Y ; 0, t] \\
& \quad=\left.\frac{1}{r !(1-t)^{r}} \Omega\left[(1-t) X Z_{r}\right] \Omega\left[(1-t) Y Z_{r}^{-1}\right] \Omega\left[(t-1) \sum_{j \neq k} \frac{z_{j}}{z_{k}}\right]\right|_{z^{0}}
\end{aligned}
$$

and

(5.34)

$$
\begin{aligned}
& \sum_{m=0}^{r} \frac{(-1)^{r-m} t^{\left(\begin{array}{c}
m \\
2
\end{array}\right)-m r}}{(t ; t)_{r-m}} \sum_{\mu: l(\mu)=m} P_{\mu}\left[X ; 0, t^{-1}\right] Q_{\mu}\left[Y ; 0, t^{-1}\right] \\
& \quad=\frac{1}{\left.r !(t-1)^{r} t^{\left(\begin{array}{c}
r \\
2
\end{array}\right)} \Omega\left[\left(1-t^{-1}\right) X Z_{r}\right] \Omega\left[\left(1-t^{-1}\right) Y Z_{r}^{-1}\right] \Omega\left[\left(t^{-1}-1\right) \sum_{j \neq k} \frac{z_{j}}{z_{k}}\right]\right|_{z^{0}} .} .
\end{aligned}
$$

For $m \geq 0$, and any alphabets $X, Y$,

$$
\begin{aligned}
& \sum_{\mu: l(\mu)=m} P_{\mu}[X ; 0, t] Q_{\mu}[Y ; 0, t] \\
& \quad=\left.\sum_{r=0}^{m} \frac{(-1)^{m-r} t^{\left(\begin{array}{c}
m-r \\
2
\end{array}\right)}}{(t ; t)_{m-r} r !(1-t)^{r}} \Omega\left[(1-t) X Z_{r}\right] \Omega\left[(1-t) Y Z_{r}^{-1}\right] \Omega\left[(t-1) \sum_{j \neq k} \frac{z_{j}}{z_{k}}\right]\right|_{z^{0}} .
\end{aligned}
$$


Proof. Macdonald shows that under the scalar product 5.2, the symmetric functions $P_{\mu}\left[Z_{r} ; q, t\right]$ and $Q_{\lambda}\left[Z_{r} ; q, t\right]$ are orthogonal when $\mu \neq \lambda$, and

$$
\begin{aligned}
& \left\langle P_{\mu}\left[Z_{r} ; q, t\right], Q_{\mu}\left[Z_{r} ; q, t\right]\right\rangle_{r, q, t}^{\prime} \\
& \quad=\Omega\left[-\frac{(1-t)\left(1-q t^{-1}\right)}{1-q} \sum_{1 \leq i<j \leq r} t^{j-i}\right] \prod_{s \in \mu} \frac{1-q^{a_{\mu}^{\prime}(s)} t^{r-l_{\mu}^{\prime}(s)}}{1-q^{a_{\mu}^{\prime}(s)+1} t^{r-l_{\mu}^{\prime}(s)-1}} .
\end{aligned}
$$

On setting $q=0$, the $\Omega$ term simplifies to

$$
\begin{aligned}
\Omega\left[-(1-t) \sum_{1 \leq i<j \leq r} t^{j-i}\right] & =\Omega\left[(t-1)\left(t^{r-1}+2 t^{r-2}+\cdots+(r-1) t\right)\right] \\
& =\Omega\left[t^{r}+t^{r-1}+\cdots+t^{2}-(r-1) t\right] \\
& =\frac{(1-t)^{r}}{(t ; t)_{r}} .
\end{aligned}
$$

Additionally, on setting $q=0$, the product term of 5.36 restricts to $s \in \mu$ for which $a_{\mu}^{\prime}(s)=0$, that is, to the cells $(i, 0)$ (for $i=0,1, \ldots, l(\mu)-1$ ) of the first column, giving

$$
\left\langle P_{\mu}\left[Z_{r} ; 0, t\right], Q_{\mu}\left[Z_{r} ; 0, t\right]\right\rangle_{r, 0, t}^{\prime}=\frac{(1-t)^{r}}{(t ; t)_{r}} \prod_{i=0}^{l(\mu)-1}\left(1-t^{r-i}\right)=\frac{(1-t)^{r}}{(t ; t)_{r-l(\mu)}}
$$

For arbitrary alphabets $X, Y$, we now evaluate

$$
\Omega_{r}[X ; Y ; t]=\left\langle\Omega\left[(1-t) X Z_{r}\right], \Omega\left[(1-t) Y Z_{r}\right]\right\rangle_{r, 0, t}^{\prime}
$$

in two ways. By expanding these as Hall-Littlewood kernels, we obtain

$$
\begin{aligned}
\Omega_{r}[X ; Y ; t] & =\left\langle\sum_{\mu} P_{\mu}[X ; 0, t] Q_{\mu}\left[Z_{r} ; 0, t\right], \sum_{\lambda} P_{\lambda}\left[Z_{r} ; 0, t\right] Q_{\lambda}[Y ; 0, t]\right\rangle_{r, 0, t}^{\prime} \\
& =\sum_{\mu} P_{\mu}[X ; 0, t] Q_{\mu}[Y ; 0, t] \cdot\left\langle Q_{\mu}\left[Z_{r} ; 0, t\right], P_{\mu}\left[Z_{r} ; 0, t\right]\right\rangle_{r, 0, t}^{\prime} \\
& =\sum_{m=0}^{r} \frac{(1-t)^{r}}{(t ; t)_{r-m}} \sum_{\mu: l(\mu)=m} P_{\mu}[X ; 0, t] Q_{\mu}[Y ; 0, t] .
\end{aligned}
$$

On the other hand, by evaluating 5.39 via 5.2 , we obtain

$$
\Omega_{r}[X ; Y ; t]=\left.\frac{1}{r !} \Omega\left[(1-t) X Z_{r}\right] \Omega\left[(1-t) Y Z_{r}^{-1}\right] \Omega\left[(t-1) \sum_{j \neq k} \frac{z_{j}}{z_{k}}\right]\right|_{z^{0}} .
$$

Equating 5.40 with 5.41 gives 5.33. On replacing $t$ by $1 / t$, using $\left(t^{-1} ; t^{-1}\right)_{k}=$ $(-1)^{k} t^{-k(k+1) / 2}(t ; t)_{k}$, and simplifying, we obtain 5.34. Finally, applying Lemma 5.1 to 5.33 gives 5.35 .

LEMMA 5.2. For $0 \leq r \leq n$,

$$
\sum_{k=0}^{r} \frac{(-1)^{k} t^{\left(\begin{array}{l}
k \\
2
\end{array}\right)}}{(t ; t)_{r-k}}\left[\begin{array}{l}
n \\
k
\end{array}\right]_{t}=\frac{t^{r n}}{(t ; t)_{r}}
$$


Proof. We specialize the " $q$-binomial theorem" [16, p. 20]

$$
(a b ; q)_{r}=\sum_{k=0}^{r}\left[\begin{array}{l}
r \\
k
\end{array}\right]_{q} b^{k}(a ; q)_{k}(b ; q)_{r-k}
$$

by setting $q=t, a=0$, and $b=t^{-n}$, and then manipulating it.

$$
\begin{aligned}
1=(0 ; t)_{r} & =\sum_{k=0}^{r}\left[\begin{array}{l}
r \\
k
\end{array}\right]_{t} t^{-n k}\left(t^{-n} ; t\right)_{r-k} \\
& =\sum_{k=0}^{r}\left[\begin{array}{l}
r \\
k
\end{array}\right]_{t} t^{-n(r-k)}\left(t^{-n} ; t\right)_{k} \\
& =\sum_{k=0}^{r}\left[\begin{array}{l}
r \\
k
\end{array}\right]_{t} t^{-n(r-k)}(-1)^{k} t^{\left(\begin{array}{c}
k \\
2
\end{array}\right)-n k} \frac{(t ; t)_{n}}{(t ; t)_{n-k}} \\
& =t^{-n r} \sum_{k=0}^{r}(-1)^{k} t^{\left(\begin{array}{c}
k \\
2
\end{array}\right)}\left[\begin{array}{l}
n \\
k
\end{array}\right]_{t} \frac{(t ; t)_{r}}{(t ; t)_{r-k}} .
\end{aligned}
$$

Multiplying by $t^{n r} /(t ; t)_{r}$ gives 5.42 .

To terminate we should mention that even the operators $\Delta_{F}$ have some surprising positivity properties. In fact, we have strong computer evidence that $\Delta_{S_{\nu}} e_{m}$ is always Schur positive. More precisely we are led to the following

Conjecture V. For any integer $m \geq 1$ and for all pairs of partitions $\nu, \lambda$ with $m \geq l(\nu)$ and $\lambda \vdash m$ we have

$$
\sum_{\mu \vdash m} \frac{S_{\nu}\left[B_{\mu}(q, t)\right] \tilde{K}_{\lambda \mu}(q, t)(1-t)(1-q) B_{\mu}(q, t) \Pi_{\mu}(q, t)}{\tilde{h}_{\mu}(q, t) \tilde{h}_{\mu}^{\prime}(q, t)} \in \mathbf{N}[q, t]
$$

\section{REFERENCES}

[1] G. ANDrews, Identities in combinatorics II: A q-analog of the Lagrange inversion theorem, Proc. Amer. Math Soc., 53 (1975), pp. 240-245.

[2] F. Bergeron, N. Bergeron, A. M. Garsia, M. Haiman, and G. Tesler, Lattice Diagram Polynomials and Extended Pieri Rules, Advances in Math., 2 (1999), pp. 244-334.

[3] F. Bergeron and A. Garsia, Science Fiction and Macdonald Polynomials, in Algebraic methods and q-special functions, R. Floreanini and L. Vinet, ed., CRM Proceedings \& Lecture Notes, AMS, 1999.

[4] M. Bousquet Mélou, F. Bergeron, and Gouyou-Beauchamps, personal communication.

[5] C. Chang, Geometric interpretation of the Macdonald polynomials and the $n$ ! conjecture, Doctoral dissertation UCSD (1998).

[6] A. Garsia, Recent Progress on the Macdonald q,t-Kostka conjecture, in Actes du $4^{e}$ Colloque sur les Séries Formelles et Combinatoire Algébrique, Publication of Lacim, Université du Québec à Montréal, 1992, pp. 249-255.

[7] A. M. Garsia, A q-analogue of the Lagrange inversion formula, Hous. J. Math., 7 (1981), pp. 205-237.

[8] A. M. Garsia and M. Haiman, A Graded Representation Model for Macdonald's Polynomials Proceedings of The National Academy, 90 (1993), pp. 3607-3610. 
[9] A. M. Garsia and M. Haiman, Orbit Harmonics and Graded Representations, To appear as a Research Monograph of Lacim, Université du Québec à Montréal.

[10] A. M. Garsia AND C. Procesi, On certain graded $S_{n}$-modules and the $q$-Kostka polynomials, Advances in Mathematics, 94 (1992), pp. 82-138.

[11] A. GARSIA AND M. HAIMAN, A remarkable $q, t$-Catalan sequence and $q$-Lagrange inversion, J. Algebraic Combin., 5:3 (1996), pp. 191-244.

[12] A. Garsia and M. Haiman, Some bigraded $S_{n}$-modules and the Macdonald q,t-Kostka coefficients, Electronic Journal of Algebraic Combinatorics, 3:2 (1996), pp. 561-620.

[13] A. Garsia, M. Haiman, and G. Tesler, Explicit Plethystic Formulas for Macdonald $q, t-$ Kostka Coefficients, in The Andrews Festschrift, Séminaire Lotharingien de Combinatoire 42, paper B42m (Web site http://www.emis.de/journals/SLC/).

[14] A. M. Garsia and J. Remmel, Plethystic Formulas and positivity for $q, t$-Kostka Coefficients, in Mathematical Essays in Honor of Gian-Carlo Rota, Progress in Mathematics 161, B. E. Sagan and R. Stanley, ed, 1998, pp. 245-262.

[15] A. M. Garsia And G. Tesler, Plethystic formulas for Macdonald q, t-Kostka coefficients, Adv. Math., 123:2 (1996), pp. 144-222.

[16] G. Gasper and M. Rahman, Basic Hypergeometric Series, (with a foreword by Richard Askey), Cambridge Univ. Press, Cambridge, 1990.

[17] I. GESSEL, A non-commutative generalization and q-analog of the Lagrange inversion formula, Amer. Math Soc. Trans. (1980), pp. 455-482.

[18] M. HAIMAN, Conjectures on the quotient ring by diagonal invariants, J. Algebraic Combinatorics, 3 (1994), pp. 17-76.

[19] M. HAIMAN, Macdonald polynomials and geometry, in New Perspectives in Algebraic Combinatorics 37, Billera et al, ed., MSRI publication, 1999, pp. 207-254.

[20] C. LenART, Lagrange Inversion and Schur Functions, Journal of Algebraic Combinatorics, to appear.

[21] I. G. Macdonald, A new class of symmetric functions, in Actes du $20^{e}$ Séminaire Lotharingien, Publ. I. R. M. A. Strasbourg, 1988, pp. 131-171 (To appear also in the the new edition of [7] above).

[22] I. G. Macdonald, Symmetric Functions and Hall Polynomials, second ed., Oxford Mathematical Monographs, Oxford Science Publications, The Clarendon Press Oxford University Press, New York, 1995.

[23] E. ReIner, A Proof of the n! Conjecture for Generalized Hooks, to appear in the Journal of Combinatorial Theory, Series A.

[24] H. WEYL, The Classical Groups, their Invariants and Representations, Princeton University Press, 1946. 